\title{
COMPLEX MONGE-AMPÈRE EQUATIONS ${ }^{1}$
}

\author{
D.H. Phong*, Jian Song ${ }^{\dagger}$, and Jacob Sturm \\ * Department of Mathematics \\ Columbia University, New York, NY 10027 \\ $\dagger$ Department of Mathematics \\ Rutgers University, Piscataway, NJ 08854 \\ $\ddagger$ Department of Mathematics \\ Rutgers University, Newark, NJ 07102
}

\begin{abstract}
This is a survey of some of the recent developments in the theory of complex Monge-Ampère equations. The topics discussed include refinements and simplifications of classical a priori estimates, methods from pluripotential theory, variational methods for big cohomology classes, semiclassical constructions of solutions of homogeneous equations, and envelopes.
\end{abstract}

\footnotetext{
${ }^{1}$ Contribution to the proceedings of the Journal of Differential Geometry Conference in honor of Professor C.C. Hsiung, Lehigh University, May 2010. Work supported in part by National Science Foundation grants DMS-07-57372, DMS-09-05873, and DMS-08-47524.
} 


\section{Contents}

1 Introduction $\quad 4$

2 Some General Perspective $\quad 5$

2.1 Geometric interpretation . . . . . . . . . . . . . . . . . 6

2.2 The method of continuity . . . . . . . . . . . . . . 7

3 A Priori Estimates: $C^{0}$ Estimates $\quad 8$

3.1 Yau's original method . . . . . . . . . . . . . . . . . . 8

3.2 Reduction to Alexandrov-Bakelman-Pucci estimates . . . . . . . . . . . . 9

3.3 Methods of pluripotential theory . . . . . . . . . . . . . . . . . . 11

4 Stability Estimates $\quad 15$

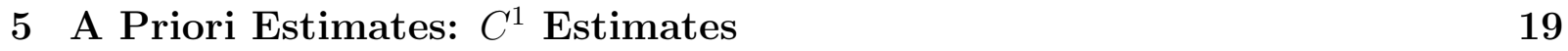

6 A Priori Estimates: $C^{2}$ Estimates $\quad 24$

7 A Priori Estimates: the Calabi identity 26

8 Boundary Regularity $\quad 27$

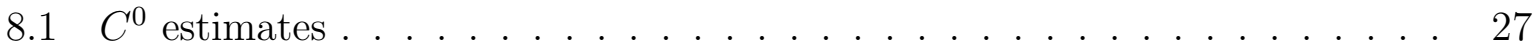

$8.2 C^{1}$ boundary estimates . . . . . . . . . . . . . . . . . 28

8.3 $C^{2}$ boundary estimates of Caffarelli-Kohn-Nirenberg-Spruck and B. Guan . 29

9 The Dirichlet Problem for the Monge-Ampère equation 30

10 Singular Monge-Ampère equations 32

10.1 Classic works . . . . . . . . . . . . . . . . . . . . 33

10.2 Monge-Ampère equations on normal projective varieties . . . . . . . . . . . 34

10.3 Positivity notions for cohomology classes . . . . . . . . . . . . . . . 37

10.4 Prescribing the Monge-Ampère measure ． . . . . . . . . . . . . . . . . . . 39

10.5 Singular KE metrics on manifolds of general type . . . . . . . . . . . . . 40

11 Variational Methods for Big Cohomology Classes 40

11.1 Finite dimensional motivation . . . . . . . . . . . . . . . . . . . 41

11.2 The infinite dimensional setting . . . . . . . . . . . . . . . . . . 44

11.3 Statement of theorems and sketch of proofs . . . . . . . . . . . . . . 45

12 Uniqueness of Solutions $\quad 48$ 
13 Semiclassical Solutions of Monge-Ampère Equations 50

13.1 Geodesics in the space of Kähler potentials . . . . . . . . . . . . . . . . 51

13.2 Geodesics from a priori estimates . . . . . . . . . . . . . . . 53

13.3 Algebraic approximations: the Tian-Yau-Zelditch theorem . . . . . . . . 55

13.4 Semi-classical constructions . . . . . . . . . . . . . . . . 57

13.5 The toric case . . . . . . . . . . . . . . . . . . . . 61

13.5.1 Bergman geodesics ................... . . . 61

13.5.2 Geodesic rays and large deviations . . . . . . . . . . . . . . 62

13.5.3 Counter-examples to regularity of higher order than $C^{1,1} \ldots$. . . . 63

13.6 The Cauchy problem for the homogeneous Monge-Ampère euation . . . . . 64

14 Envelopes and the Perron Method $\quad 65$

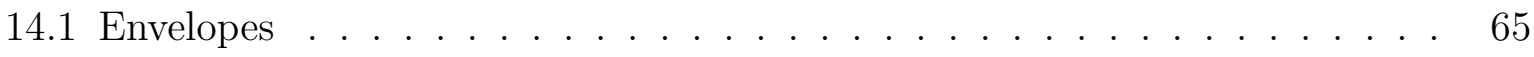

14.2 Envelopes with integral conditions . . . . . . . . . . . . . . . 69

15 Further Developments $\quad 72$

$\begin{array}{ll}\text { A Plurisubharmonic functions } & \mathbf{7 4}\end{array}$

A.1 The exponential estimate . . . . . . . . . . . . . . . . 74

A.2 Regularization of plurisubharmonic functions . . . . . . . . . . . . . . 74

A.3 The comparison principle . . . . . . . . . . . . . . . . . . 75 


\section{Introduction}

Monge-Ampère equations are second-order partial differential equations whose leading term is the determinant of the Hessian of a real unknown function $\varphi$. As such, they are arguably the most basic of fully non-linear equations. The Hessian is required to be positive or at least non-negative, so the equations are elliptic or degenerate elliptic. Monge-Ampère equations can be divided into real or complex, depending on whether $\varphi$ is defined on a real or complex manifold. In the real case, the Hessian is $\nabla_{j} \nabla_{k} \varphi$, so the positivity of the Hessian is a convexity condition. In the complex case, the Hessian is $\partial_{j} \partial_{\bar{k}} \varphi$, and its positivity is rather a plurisubharmonicity condition. Unlike convex functions, plurisubharmonic functions can have singularities, and this accounts for many significant differences between the theories of real and complex Monge-Ampère equations. In these lectures, we shall concentrate on the complex case.

The foundations of an existence and regularity theory for complex Monge-Ampère equations in the elliptic case, with smooth data, were laid by Yau [Y78] and Caffarelli, Kohn, Nirenberg, and Spruck [CNS, CKNS]. In [Y78], a complete solution was given for the Calabi conjecture, which asserts the existence of a smooth solution to the equation

$$
\left(\omega_{0}+\frac{i}{2} \partial \bar{\partial} \varphi\right)^{n}=e^{f(z)} \omega_{0}^{n},
$$

on a compact $n$-dimensional Kähler manifold $\left(X, \omega_{0}\right)$ without boundary, where $f(z)$ is a given smooth function satisfying the necessary condition $\int_{X} e^{f} \omega_{0}^{n}=\int_{X} \omega_{0}^{n}$. The solution was by the method of continuity, and the key estimates for the $C^{0}$ norms of $\varphi, \Delta \varphi$, and $\nabla_{j} \nabla_{\bar{k}} \nabla_{l} \varphi$ were formulated and derived there. In [CKNS], a complete solution was given for the Dirichlet problem

$$
\operatorname{det}\left(\partial_{j} \partial_{\bar{k}} \varphi\right)=F(z, \varphi) \text { on } D, \quad \varphi=\varphi_{b} \text { on } \partial D,
$$

where $D$ is a smooth, bounded, strongly pseudoconvex domain in $\mathbf{C}^{n}, F \in C^{\infty}(\bar{D} \times \mathbf{R})$, $F(z, \varphi)>0, F_{\varphi}(z, \varphi) \geq 0$, and $\varphi_{b} \in C^{\infty}(\partial D)$. A crucial ingredient of the existence and regularity developed there is the $C^{0}$ boundary estimates for the second order derivatives and their modulus of continuity.

In his paper [Y78], Yau also began an existence and regularity theory for singular complex Monge-Ampère equations on Kähler manifolds. Here the term "singular" should be interpreted in a broad sense. It encompasses situations where the right hand side may be degenerate or have singularities [Y78], or where the manifold $X$ may not be compact or have singularities [CY80, MY83, CY86, TY86], or where the boundary condition may be infinite [CY80]. Such extensions were required by geometric applications, and many important results were obtained, of which the references we just gave are just a small sample (see e.g. [TY90, TY91, K83, W08, LYZ], and especially [Y93, Y94, Y96] and references therein). 
The last fifteen years or so have witnessed remarkable progresses in the theory of singular Monge-Ampère equations. A particularly strong impetus was provided by related problems from the minimal model program in algebraic geometry ([EGZ, ST08, TZ, BEGZ]) and from the problem of finding metrics of constant scalar curvature in a given Kähler class (see [Y93, T97, D02] and [PS08] for a survey). The solutions in these problems are often inherently singular, and thus they must be understood in a generalized sense. The foundations of a theory of generalized solutions for the complex Monge-Ampère equation or pluripotential theory - had been laid out by Bedford and Taylor in [BT76, BT82]. There they constructed Monge-Ampère measures for bounded potentials and capacities, established monotonicity theorems for their convergence, and obtained generalized solutions of the Dirichlet problem for degenerate right hand sides by the Perron method. A key catalyst for several of the recent progresses is the theorem of Kolodziej [K98], based on pluripotential theory, which provided $C^{0}$ estimates for Monge-Ampère equations with right hand sides in $L^{p}$ for any $p>1$. Other important ingredients have been the extensions of pluripotential theory to unbounded potentials ([GZ, BBGZ, Ceg, B06, CG] and references therein), the Tian-Yau-Zelditch theorem [Y93, T90a, Z, Cat, L] on approximations of smooth metrics by Fubini-Study metrics [PS06, PS07, PS09b, SZ07, SZ10, RZ08, RZ10a, RZ10b], and refinements and extensions [Gb, B09b, PS09a, PS09c, GL, Gp, Ch, TW1, TW2, DK] of the classic estimates in [Y78, CKNS].

The main goal of this paper is to survey some of the recent progresses. There have been many of them, and the theory is still in full flux. While definitive answers may not yet be available to many questions, we thought it would be useful to gather here in one place, for the convenience of students and newcomers to the field, some of what is known. It was not possible to be comprehensive, and our selection of material necessarily reflects our own limitations. At the same time, we hope that the survey would be useful to a broad audience of people with relatively little familiarity with complex Monge-Ampère equations, and we have provided reasonably complete derivations in places, when the topics are of particular importance or the literature not easily accessible. Each of us has lectured on parts of this paper at our home institutions, and at various workshops. In particular, the first-named author spoke at the 2010 conference at Lehigh University in honor of Professor C.C. Hsiung, one of the founders of the Journal of Differential Geometry. We would like to contribute this paper to the volume in his honor.

\section{Some General Perspective}

Let $\left(X, \omega_{0}\right)$ be a compact Kähler manifold. We consider complex Monge-Ampère equations of the form

$$
\left(\omega_{0}+\frac{i}{2} \partial \bar{\partial} \varphi\right)^{n}=F(z, \varphi) \omega_{0}^{n}
$$


where $F(z, \varphi)$ is a non-negative function. The solution $\varphi$ is required to be $\omega_{0}$-plurisub harmonic, that is, $\varphi \in P S H\left(X, \omega_{0}\right)$, with

$$
P S H\left(X, \omega_{0}\right)=\left\{\varphi: X \rightarrow[-\infty, \infty) ; \varphi \text { is upper semicontinuous, } \omega_{\varphi} \equiv \omega_{0}+\frac{i}{2} \partial \bar{\partial} \varphi \geq 0\right\} \text {. }
$$

We shall consider both the case of $X$ compact without boundary, and the case of $\bar{X}$ compact with smooth boundary $\partial X$, in which case we also impose a Dirichlet condition $\varphi=\varphi_{b}$, where $\varphi_{b} \in C^{\infty}(\partial X)$ is a given function.

\subsection{Geometric interpretation}

Equations of the form (2.1) are fundamentally geometric in nature. The form $\omega_{\varphi}$ can be viewed as a form in the same cohomology class as $\omega_{0}$. It defines a regular Kähler metric when it is $>0$, or a Kähler metric with degeneracies when it does have zeroes. It is well-known that the Ricci curvature form Ricci $\left(\omega_{\varphi}\right)$ of a Kähler form $\omega_{\varphi}$ is given by

$$
\operatorname{Ricci}\left(\omega_{\varphi}\right)=-\frac{i}{2} \partial \bar{\partial} \log \omega_{\varphi}^{n}
$$

Thus the equation (2.1) is just an equation for a possibly degenerate metric $\omega_{\varphi}$ in the same Kähler class as $\omega_{0}$, satisfying a given constraint on its volume form $\omega_{\varphi}^{n}$ or, equivalently upon differentiation, a given constraint on its Ricci curvature $\operatorname{Ricci}\left(\omega_{\varphi}\right)$.

The modern theory of complex Monge-Ampère equations began with the following two fundamental theorems, due respectively to Yau [Y78] and to Yau [Y78] and Aubin [A].

Theorem 1 Let $\left(X, \omega_{0}\right)$ be a compact Kähler manifold without boundary, and let $F(z)=$ $e^{f(z)}$, where $f(z)$ is a smooth function satisfying the condition

$$
\int_{X} e^{f} \omega_{0}^{n}=\int_{X} \omega_{0}^{n}
$$

Then the equation (2.1) admits a smooth solution $\varphi \in P S H\left(X, \omega_{0}\right)$, unique up to an additive constant.

Theorem 2 Let $\left(X, \omega_{0}\right)$ be a compact Kähler manifold without boundary, and let $F(z, \varphi)=$ $e^{f+\varphi}$ where $f(z)$ is a smooth function. Then the equation (2.1) admits a unique smooth solution $\varphi \in P S H\left(X, \omega_{0}\right)$.

Geometrically, Theorem 1 provides a solution of the Calabi conjecture, which asserts that, on a compact Kähler manifold $X$ with $c_{1}(X)=0$, there is a unique metric $\omega_{\varphi}$ with $\operatorname{Ricci}\left(\omega_{\varphi}\right)=0$ in any Kähler class $\left[\omega_{0}\right]$. Indeed, the formula (2.2) shows that the Ricci form of any Kähler metric must be in $c_{1}(X)$. The assumption that $c_{1}(X)=0$ implies that 
$\operatorname{Ricci}\left(\omega_{0}\right)=\frac{i}{2} \partial \bar{\partial} f$ for some smooth function $f(z)$. It is now readily verified, by taking $F(z)=e^{f(z)}$ in the equation (2.1) and taking $\frac{i}{2} \partial \bar{\partial}$ of both sides, that the solution of (2.1) satisfies the condition

$$
\operatorname{Ricci}\left(\omega_{\varphi}\right)=0
$$

Similarly, Theorem 2 implies the existence of a Kähler-Einstein metric with negative curvature on any compact Kähler manifold $X$ with $c_{1}(X)<0$. In this case, since $c_{1}(X)<$ 0 , we can choose a Kähler form $\omega_{0}$ in the cohomology class $-c_{1}(X)$. But the Ricci curvature form Ricci $\left(\omega_{0}\right)$ is still in $c_{1}(X)$, and thus there is a smooth function $f(z)$ with Ricci $\left(\omega_{0}\right)+$ $\omega_{0}=\frac{i}{2} \partial \bar{\partial} f$. Taking $F(z, \varphi)=e^{f+\varphi}$ in the equation (2.1) and taking again $i \partial \bar{\partial}$ of both sides, we see that the solution of (2.1) satisfies now the Kähler-Einstein condition

$$
\operatorname{Ricci}\left(\omega_{\varphi}\right)=-\omega_{\varphi}
$$

We note that the Kähler-Einstein problem for compact Kähler manifolds $X$ with $c_{1}(X)>0$ is still open at this time, despite a lot of progress [TY87, Si, N, T90b, T97, D10, D11a, D11b, CDa, CDb]. A well-known conjecture of Yau [Y93] asserts the equivalence between the existence of such a metric on $X$ and the stability of $X$ in geometric invariant theory. This can be reduced, just as above for the cases $c_{1}(X)=0$ and $c_{1}(X)<0$, to a complex Monge-Ampère equation of the form $(2.1)$, but with $F(z, \varphi)=e^{f(z)-\varphi}$. Thus the conjecture of Yau asserts the equivalence between the solvability of a complex Monge-Ampère equation and a global, algebraic-geometric, condition. Clearly, bringing the algebraic-geometric conditions into play in the solution of a non-linear partial differential equation is an important and challenging problem. The two major successes in this direction are the theorem of Donaldson-Uhlenbeck-Yau [D87, UY], on the equivalence between the existence of a Hermitian-Einstein metric on a holomorphic vector bundle $E \rightarrow(X, \omega)$ and the Mumford-Takemoto stability of $E$, and the recent results of Donaldson [D08] on the equivalence between the existence of metrics of constant scalar curvature on toric 2-folds and their K-stability. However, there are still many unanswered questions in this direction.

\subsection{The method of continuity}

The original proof of Theorems 1 and 2 is by the method of continuity, and this has remained a prime method for solving complex Monge-Ampère equations to this day. In this method, the equation to be solved is deformed continuously to an equation which we know how to solve. For example, one introduces for Theorem 1 the deformation,

$$
\left(\omega_{0}+\frac{i}{2} \partial \bar{\partial} \varphi\right)^{n}=\frac{\int_{X} \omega_{0}^{n}}{\int_{X} e^{t f(z)} \omega_{0}^{n}} e^{t f(z)} \omega_{0}^{n}, \quad 0 \leq t \leq 1
$$

and for Theorem 2 the deformation,

$$
\left(\omega_{0}+\frac{i}{2} \partial \bar{\partial} \varphi\right)^{n}=e^{t f(z)+\varphi} \omega_{0}^{n}, \quad 0 \leq t \leq 1 .
$$


These equations admit trivially the smooth solution $\varphi=0$ at $t=0$. It is not difficult to show, by the implicit function theorem, that the set of parameters $t$ for which the equation is solvable is open. So to show that this set is the full interval $[0,1]$ reduces to show that it is closed. This in turn reduces to the proof of a priori estimates for the solutions $\varphi$, assuming that they already exist and are smooth.

\section{A Priori Estimates: $C^{0}$ Estimates}

We begin by discussing $C^{0}$ estimates for the most basic complex Monge-Ampère equation. Let $\left(X, \omega_{0}\right)$ be a compact Kähler manifold without boundary, and consider the equation

$$
\left(\omega_{0}+\frac{i}{2} \partial \bar{\partial} \varphi\right)^{n}=F(z) \omega_{0}^{n}
$$

for a smooth function $\varphi$ satisfying the condition $\varphi \in P S H\left(X, \omega_{0}\right)$, with $F(z)$ a smooth

strictly positive function. Since the equation is invariant under shifts of $\varphi$ by constants, we may assume that $\sup _{X} \varphi=0$. It is well-known that all functions in $P S H\left(X, \omega_{0}\right)$ satisfy an exponential integrability condition, and hence their $L^{p}$ norms are all uniformly bounded by constants depending only on the Kähler class $\left[\omega_{0}\right]$ and on $p$, for any $1 \leq p<\infty$ (see e.g. Appendix A). But the $L^{\infty}$, or $C^{0}$ estimate, is fundamentally different. In this section, we discuss several methods for obtaining $C^{0}$ estimates.

\subsection{Yau's original method}

Yau's original method was by Moser iteration. Set $\psi=\sup _{X} \varphi-\varphi+1 \geq 1$ and let $\alpha \geq 0$. Since $(F-1) \omega_{0}^{n}=\left(\omega_{0}+\frac{i}{2} \partial \bar{\partial} \varphi\right)^{n}-\omega_{0}^{n}=\frac{i}{2} \partial \bar{\partial} \varphi \sum_{j=0}^{n-1}\left(\omega_{0}+\frac{i}{2} \partial \bar{\partial} \varphi\right)^{n-1-j} \omega_{0}^{j}$, we find, after multiplying by $\psi^{\alpha+1}$ and integrating by parts.

$$
\int_{X} \psi^{\alpha+1}(F-1) \omega_{0}^{n}=(\alpha+1) \sum_{j=0}^{n-1} \int_{X} \psi^{\alpha} i \partial \psi \wedge \bar{\partial} \psi\left(\omega_{0}+\frac{i}{2} \partial \bar{\partial} \varphi\right)^{n-1-j} \omega_{0}^{j} .
$$

All the integrals on the right hand side are positive. Keeping only the contribution with $j=n-1$, we obtain

$$
\begin{aligned}
\left|\int_{X} \psi^{\alpha+1}(F-1) \omega_{0}^{n}\right| & \geq(\alpha+1) \int_{X} \psi^{\alpha} i \partial \psi \wedge \bar{\partial} \psi \omega_{0}^{n-1} \\
& =\frac{(\alpha+1)}{2\left(\frac{\alpha}{2}+1\right)^{2}} \int_{X} i \partial\left(\psi^{\frac{\alpha}{2}+1}\right) \wedge \bar{\partial}\left(\psi^{\frac{\alpha}{2}+1}\right) \wedge \omega_{0}^{n-1}
\end{aligned}
$$

and hence, with $C_{1}$ depending only on $\|F\|_{L^{\infty}}$, and all norms and covariant derivatives with respect to the metric $\omega_{0}$,

$$
\left\|\nabla\left(\psi^{\frac{\alpha}{2}+1}\right)\right\|^{2} \leq C_{1} \frac{n\left(\frac{\alpha}{2}+1\right)^{2}}{\alpha+1} \int_{X} \psi^{\alpha+1} \omega_{0}^{n} .
$$


On the other hand, the Sobolev inequality asserts that

$$
\|u\|_{L^{\frac{2 n}{n-1}}}^{2} \leq C_{2}\left(\|\nabla u\|_{L^{2}}^{2}+\|u\|_{L^{2}}^{2}\right)
$$

with $C_{2}$ the Sobolev constant of $\left(X, \omega_{0}\right)$. Applied to $u=\psi^{\frac{p}{2}}$, it can be expressed as

$$
\|\psi\|_{L^{p \beta}}^{p} \leq C_{2}\left(\left\|\nabla\left(\psi^{\frac{p}{2}}\right)\right\|_{L^{2}}^{2}+\|\psi\|_{L^{p}}^{p}\right)
$$

with $\beta=\frac{n}{n-1}>1$. Setting $p=\alpha+2$, and applying the inequality (3.4), we find

$$
\|\psi\|_{L^{p \beta}} \leq\left(C_{3} p\right)^{\frac{1}{p}}\|\psi\|_{L^{p}}, \quad p \geq 2
$$

with a constant $C_{3}$ depending only on $n,\|F\|_{L^{\infty}}$, and the Sobolev constant of $\left(X, \omega_{0}\right)$. We can iterate $p \rightarrow p \beta \rightarrow \cdots \rightarrow p \beta^{k}$ and get

$$
\log \|\psi\|_{L^{\infty}} \leq \sum_{k=0}^{\infty} \frac{\log \left(C_{3} p \beta^{k}\right)}{p \beta^{k}}+\log \|\psi\|_{L^{p}}=C_{4, p}+\log \|\psi\|_{L^{p}}
$$

An a priori bound for $\|\psi\|_{L^{p}}$ for any fixed finite $p$ can be obtained from the exponential estimate for plurisubharmonic functions in Appendix A. Alternatively, if we apply Moser iteration instead to the function $\varphi$ normalized to have average 0 , we can obtain an a priori bound for $\|\varphi\|_{L^{2}}$ from the analogue of (3.4) by taking $\alpha=0$, and applying the Poincaré inequality to the left hand side. Either way gives

Theorem 3 Let $\varphi$ be a smooth solution of the equation (3.1) on a compact Kähler manifold $\left(X, \omega_{0}\right)$ without boundary, $F>0$, and $\varphi \in P S H\left(X, \omega_{0}\right)$. Then $\|\psi\|_{L^{\infty}(X)}$ is bounded by a constant depending only on $n$, an upper bound for $\|F\|_{L^{\infty}(X)}$, and the Kähler form $\omega_{0}$. The dependence on the Kähler form $\omega_{0}$ can be stated more precisely as a dependence on the Sobolev constant and the Poincaré constant of $\omega_{0}$, or on the exponential bound for $\omega_{0}$.

The Moser iteration method is now widely used in the study of Monge-Ampère and other non-linear equations. An important variant has been introduced by Weinkove [W], where the Moser iteration is applied to $e^{\varphi}$ instead of $\varphi$. Applications of this variant are in [SW, ST06, TWY].

\subsection{Reduction to Alexandrov-Bakelman-Pucci estimates}

It was suggested early on by Cheng and Yau that the Alexandrov-Bakelman-Pucci estimate can be applied to the complex Monge-Ampère equation. They did not publish their work, but a detailed account was subsequently provided by Bedford [B] and Cegrell and Persson $[\mathrm{CP}]$. Using the Alexandrov-Bakelman-Pucci estimate, Blocki [B11a] gives the following proof of the $C^{0}$ estimate. This proof is of particular interest as it is almost a local argument. We follow closely Blocki's presentation. 
Let $D$ be any bounded domain in $\mathbf{C}^{n}, u \in C^{2}(\bar{D}), u_{\bar{k} j} \geq 0$, and $u=0$ on $\partial D$. Then

$$
\|u\|_{C^{0}} \leq C\left\|\operatorname{det} u_{\bar{k} j}\right\|_{L^{2}}^{\frac{1}{n}}
$$

where $C=C(n, \operatorname{diam} D)$ depends only on the diameter of $D$ and the dimension $n$. To see this, we apply the ABP estimate (from [GT], Lemma 9.2) to get

$$
\|u\|_{C^{0}} \leq c_{n}(\operatorname{diam} D)\left(\int_{\Gamma} \operatorname{det} D^{2} u\right)^{\frac{1}{2 n}}
$$

where $\Gamma$ is the contact set, defined by

$$
\Gamma=\{z \in D ; u(w) \geq u(z)+\langle D u(z), w-z\rangle, \text { for all } w \in D\} .
$$

On the contact set $\Gamma$, the function $u$ satisfies $D^{2} u \geq 0$, and for such functions, we have the following inequality between the determinants of the real and complex Hessians,

$$
\operatorname{det} u_{\bar{k} j} \geq 2^{-n}\left(\operatorname{det} D^{2} u\right)^{\frac{1}{2}}
$$

This proves the estimate (3.9).

Let now $z \in D, h>0$, and define the sublevel set $S(z, h)$ by

$$
S(z, h)=\{w \in D ; u(w)<u(z)+h\} .
$$

If $S(z, h) \subset \subset D$, then applying the previous inequality to $S(z, h)$ instead of $D$ gives

$$
\begin{aligned}
\|u-u(z)-h\|_{C^{0}(S(z, h))} & \leq C(n, \operatorname{diam} D)\left\|\operatorname{det} u_{\bar{k} j}\right\|_{L^{2}(S(z, h))}^{\frac{1}{n}} \\
& \leq C(n, \operatorname{diam} D)|S(z, h)|^{\frac{1}{2 n q}}\left\|\operatorname{det} u_{\bar{k} j}\right\|_{L^{2 p}}^{\frac{1}{n}}
\end{aligned}
$$

for any $p>1, \frac{1}{p}+\frac{1}{q}=1$. In particular, we obtain the following lower bound for $|S(z, h)|$

$$
h \leq C(n, \operatorname{diam} D)|S(z, h)|^{\frac{1}{2 q n}}\left\|\operatorname{det} u_{\bar{k} j}\right\|_{L^{2 p}}^{\frac{1}{n}} .
$$

On the other hand, we have the following easy upper bound for $|S(z, h)|$,

$$
|S(z, h)|(-u(z)-h) \leq \int_{S(z, h)}(-u) \leq\|u\|_{L^{1}(D)}
$$

If we choose $z$ to be the minimum point for $u$, and eliminate $|S(z, h)|$ between the two inequalities, we obtain a lower bound for $u$ in terms of $h$ and $h^{-1}$.

This can be applied to the $C^{0}$ estimate for the Monge-Ampère equation $\left(\omega_{0}+\frac{i}{2} \partial \bar{\partial} \varphi\right)^{n}=$ $F(z) \omega_{0}^{n}$ on a compact Kähler manifold $\left(X, \omega_{0}\right)$. Let $z$ be the minimum point for $\varphi$ on $X$, and let $K(w, \bar{w})$ be a Kähler potential for $\omega_{0}$ in a neighborhood of $z$. By adding a negative constant and shifting $K(w, \bar{w})$ by the real part of a second order polynomial in $w$ if 
necessary, we can assume that $K(w, \bar{w}) \leq 0$ in a ball $B(z, 2 r)$ around $z, K(w, \bar{w}) \geq K(0)+h$ for $r \leq|w| \leq 2 r$, and $K(w, \bar{w})$ attains its minimum in $B(z, 2 r)$ at 0 . The constant $h>0$ depends only on the Kähler form $\omega_{0}$. Then the function $u=K+\varphi$ attains its minimum in $B(z, 2 r)$ at $z$, and the corresponding set $S(z, h) \subset B(z, 2 r) \backslash B(z, r)$ has compact closure. By the preceding inequalities, we obtain a lower bound for $u(z)$, depending only on $\omega_{0}$ and the $L^{2 p}$ norm of $F$, for any $p>1$. Thus

Theorem 4 Let the setting be the same as in Theorem 3. Then for any $p>1,\|\varphi\|_{L^{\infty}(X)}$ can be bounded by a constant depending only on $n$, an upper bound for $\|F\|_{L^{2 p}(X)}$, and the Kähler form $\omega_{0}$.

\subsection{Methods of pluripotential theory}

A third method for $C^{0}$ estimates was introduced by Kolodziej [K98]. This method combines the classic approach of De Giorgi with modern techniques of pluripotential theory. It produces $C^{0}$ bounds even when the right hand side $F$ is only in $L^{p}(X)$ for some $p>1$. As shown by Eyssidieux, Guedj, and Zeriahi [EGZ, EGZ09] and Demailly and Pali [DP], it can also be extended to a family setting, where the background Kähler form $\omega_{t}$ is allowed to degenerate to a closed form $\chi$ which is just non-negative. Other family versions of Kolodziej's $C^{0}$ estimates are in [KT, DZ, TZ]. As we shall see later, such family versions are important for the study of singular Kähler-Einstein metrics and Monge-Ampère equations on complex manifolds with singularities.

Let $\left(X, \omega_{0}\right)$ be a compact Kähler manifold. Let $\chi \geq 0$ be a $C^{\infty}$ closed semi-positive $(1,1)$-form which is not identically 0 . Set

$$
\omega_{t}=\chi+(1-t) \omega_{0}, \quad t \in(0,1)
$$

and let $\left[\omega_{t}^{n}\right]=\int_{X} \omega_{t}^{n}$. Consider the equation

$$
\left(\omega_{t}+\frac{i}{2} \partial \bar{\partial} \varphi_{t}\right)^{n}=F_{t} \omega_{t}^{n}
$$

for some strictly positive function $F_{t}$ and $\varphi_{t} \in P S H\left(X, \omega_{t}\right) \cap L^{\infty}(X)$. Then we have the following family version of the $C^{0}$ estimates of Kolodziej [K98], due to Eyssidieux-GuedjZeriahi [EGZ, EGZ09] and Demailly-Pali [DP]:

Theorem 5 Let $A>0$ and and $p>1$. Assume that $\chi \leq A \omega_{0}$ and $\frac{1}{\left[\omega_{t}^{n}\right]} \frac{\omega_{t}^{n}}{\omega_{0}^{n}} \leq A$. Assume also that the functions $F_{t}$ are in $L^{p}\left(X, \omega_{t}^{n}\right)$ and that

$$
\frac{1}{\left[\omega_{t}^{n}\right]} \int_{X} F_{t}^{p} \omega_{t}^{n} \leq A^{p}<\infty
$$

for all $t \in(0,1)$. Normalize $\varphi_{t}$ so that $\sup _{X} \varphi_{t}=0$. Then there exists a constant $C>0$, depending only on $n, \omega_{0}$ and $A$, so that

$$
\sup _{t \in[0,1)}\left\|\varphi_{t}\right\|_{L^{\infty}(X)} \leq C
$$


Proof. Recall the notion of capacity of a Borel set $E$ with respect to a Kähler form $\omega$,

$$
\operatorname{Cap}_{\omega}(E)=\sup \left\{\int_{E}\left(\omega+\frac{i}{2} \partial \bar{\partial} u\right)^{n} ; u \in P S H(X, \omega), 0 \leq u \leq 1\right\} .
$$

Set

$$
f_{t}(s)=\left(\frac{\operatorname{Cap}_{\omega_{t}}\left(\varphi_{t}<-s\right)}{\left[\omega_{t}^{n}\right]}\right)^{\frac{1}{n}} .
$$

It suffices to show that there exists $s_{\infty}<\infty$ independent of $t$ so that

$$
f_{t}(s)=0 \quad \text { for } s>s_{\infty} .
$$

Since $f_{t}(s)^{n} \geq \frac{1}{\left[\omega_{t}^{n}\right]} \int_{\varphi<-s} \omega_{t}^{n}$, it would follow that $\varphi_{t} \geq-s_{\infty}$ a.e. with respect to the measure $\omega_{t}^{n}$, and since $\varphi_{t}$ is upper semi-continuous, that $\varphi_{t} \geq-s_{\infty}$ everywhere. The following classic lemma of De Giorgi provides sufficient conditions for the existence of $s_{\infty}$ :

Lemma 1 Let $f: \mathbf{R}^{+} \rightarrow \mathbf{R}^{+}$satisfy the following conditions:

(a) $f$ is right-continuous;

(b) $f$ decreases to 0 ;

(c) There exist positive constants $\alpha, A_{\alpha}$ so that for all $s \geq 0$ and all $0 \leq r \leq 1$, we have

$$
r f(s+r) \leq A_{\alpha} f(s)^{1+\alpha} .
$$

Then there exists $s_{\infty}$, depending only on $\alpha, A_{\alpha}$ and the smallest value $s_{0}$ for which we have $f\left(s_{0}\right)^{\alpha} \leq\left(2 A_{\alpha}\right)^{-1}$ so that $f(s)=0$ for $s>s_{\infty}$. In fact, we can take $s_{\infty}=s_{0}+2 A_{\alpha}(1-$ $\left.2^{-\alpha}\right)^{-1} f\left(s_{0}\right)^{\alpha}$.

We shall show that the above functions $f_{t}(s)$ satisfy the conditions of Lemma 1 . The right-continuity (a) of the function $f_{t}(s)$ is a consequence of the fact that, for any Kähler form $\omega$, and any sequence of increasing sequence of Borel sets $E_{j} \subset E_{j+1}$, we have $\operatorname{Cap}_{\omega}\left(\cup_{j=1}^{\infty} E_{j}\right)=\lim _{j \rightarrow \infty} \operatorname{Cap}_{\omega}\left(E_{j}\right)$. Clearly $f_{t}(s)$ decreases as $s$ increases. In fact, it does so uniformly to 0 in $t$ as is shown by the following lemma:

Lemma 2 There exists a constant $C$ depending only on $\omega_{0}$ and an upper bound $A$ for $\chi$ so that

$$
f_{t}(s)^{n} \leq C s^{-1} .
$$

Proof of Lemma 2. Let $u \in P S H\left(X, \omega_{t}\right)$. Then

$$
\begin{aligned}
\int_{\varphi_{t}<-s}\left(\omega_{t}+\frac{i}{2} \partial \bar{\partial} u\right)^{n} & \leq \frac{1}{s} \int_{X}\left(-\varphi_{t}\right)\left(\omega_{t}+\frac{i}{2} \partial \bar{\partial} u\right)^{n} \\
& =\frac{1}{s} \int_{X}\left(-\varphi_{t}\right) \omega_{t}^{n}+\frac{1}{s} \int_{X}\left(-\varphi_{t}\right) \frac{i}{2} \partial \bar{\partial} u \sum_{j=0}^{n-1} \omega_{t}^{j}\left(\omega_{t}+\frac{i}{2} \partial \bar{\partial} u\right)^{n-1-j}
\end{aligned}
$$


Writing $\omega_{t}^{n} \leq A\left[\omega_{t}^{n}\right] \omega_{0}^{n}$, and noting that $P S H\left(X, \omega_{t}\right) \subset P S H\left(X,(A+1) \omega_{0}\right)$, we can bound the first integral on the right hand side by $C\left[\omega_{t}^{n}\right]$, in view of Theorem A.1 on exponential estimates for plurisubharmonic functions. The other integrals can be re-expressed as

$$
\begin{aligned}
& \int_{X} \varphi_{t} \frac{i}{2} \partial \bar{\partial} u \omega_{t}^{j}\left(\omega_{t}+\frac{i}{2} \partial \bar{\partial} u\right)^{n-1-j}=-\int_{X} \frac{i}{2} \partial \bar{\partial} \varphi_{t} u \omega_{t}^{j}\left(\omega_{t}+\frac{i}{2} \partial \bar{\partial} u\right)^{n-1-j} \\
& =-\int_{X} u\left(\omega_{t}+\frac{i}{2} \partial \bar{\partial} \varphi_{t}\right) \omega_{t}^{j}\left(\omega_{t}+\frac{i}{2} \partial \bar{\partial} u\right)^{n-1-j}+\int_{X} u \omega_{t}^{j+1}\left(\omega_{t}+\frac{i}{2} \partial \bar{\partial} u\right)^{n-1-j} .
\end{aligned}
$$

For $0 \leq u \leq 1$, we can write

$$
\left|\int_{X} u\left(\omega_{t}+\frac{i}{2} \partial \bar{\partial} \varphi_{t}\right) \omega_{t}^{j}\left(\omega_{t}+\frac{i}{2} \partial \bar{\partial} u\right)^{n-1-j}\right| \leq \int_{X}\left(\omega_{t}+\frac{i}{2} \partial \bar{\partial} \varphi_{t}\right) \omega_{t}^{j}\left(\omega_{t}+\frac{i}{2} \partial \bar{\partial} u\right)^{n-1-j}=\left[\omega_{t}^{n}\right]
$$

and similarly for the other integral. Thus we obtain an upper bound $C\left[\omega_{t}^{n}\right]$, and taking the supremum in $u$ establishes the desired inequality. Q.E.D.

It remains to establish the property $(\mathrm{c})$ for $f_{t}(s)$. For this, we need the following two propositions:

Lemma 3 Let $\varphi \in P S H(X, \omega) \cap L^{\infty}(X)$. Then for all $s>0,0 \leq r \leq 1$,

$$
r^{n} \operatorname{Cap}_{\omega}(\varphi<-s-r) \leq \int_{\varphi<-s}\left(\omega+\frac{i}{2} \partial \bar{\partial} \varphi\right)^{n} .
$$

Lemma 4 There exist constants $\delta, C>0$ so that for any open set $E \subset X$, and any $t \in[0,1)$, we have

$$
\frac{1}{\left[\omega_{t}^{n}\right]} \int_{E} \omega_{t}^{n} \leq C \exp \left[-\delta\left(\frac{\left[\omega_{t}^{n}\right]}{\operatorname{Cap}_{\omega_{t}}(E)}\right)^{1 / n}\right] .
$$

Assuming these two lemmas for the moment, we can readily establish the inequality (c) in Lemma 1. For $\alpha>0$ we have

$$
\begin{aligned}
{\left[r f_{t}(s+r)\right]^{n} } & =r^{n} \frac{\operatorname{Cap}_{\omega}\left(\varphi_{t}<-s-r\right)}{\left[\omega_{t}^{n}\right]} \leq \frac{1}{\left[\omega_{t}^{n}\right]} \int_{\varphi_{t}<-s}\left(\omega+\frac{i}{2} \partial \bar{\partial} \varphi_{t}\right)^{n} \\
& =\frac{1}{\left[\omega_{t}^{n}\right]} \int_{\varphi_{t}<-s} F_{t} \omega_{t}^{n} \leq\left(\frac{1}{\left[\omega_{t}^{n}\right]} \int_{\varphi_{t}<-s} F_{t}^{p} \omega_{t}^{n}\right)^{\frac{1}{p}}\left(\frac{1}{\left[\omega_{t}^{n}\right]} \int_{\varphi_{t}<-s} \omega_{t}^{n}\right)^{\frac{1}{q}} \\
& \leq A \exp \left(-\frac{\delta}{q}\left[\frac{\left[\omega_{t}^{n}\right]}{\operatorname{Cap}_{\omega}\left(\varphi_{t}<-s\right)}\right]^{\frac{1}{n}}\right) \leq A_{\alpha} f^{(1+\alpha) n}
\end{aligned}
$$

It remains to prove the two lemmas. Let $u \in P S H(X, \omega)$ with $0 \leq u \leq 1$, and write

$$
\begin{aligned}
r^{n} \int_{\varphi<-s-r}\left(\omega+\frac{i}{2} \partial \bar{\partial} u\right)^{n} & =\int_{\varphi<-s-r}\left(r \omega+\frac{i}{2} \partial \bar{\partial} r u\right)^{n} \\
& \leq \int_{\varphi<-s-r+r u}\left(\omega+\frac{i}{2} \partial \bar{\partial}(r u-s-r)\right)^{n} \\
& \leq \int_{\varphi<-s-r+r u}\left(\omega+\frac{i}{2} \partial \bar{\partial} \varphi\right)^{n}
\end{aligned}
$$


where we have applied the comparison principle. Since $-r+r u$ is negative, this last integral is bounded by the integral over the larger region $\{\varphi<-s\}$, and Lemma 3 is proved. The next lemma requires some properties of global extremal functions [GZ, Ze]:

Lemma 5 Let $E \subset X$ be an open set, and define its global extremal function $\psi_{E, \omega}$ as the upper semi-continuous envelope of the following function $\tilde{\psi}_{E, \omega}$,

$$
\tilde{\psi}_{E, \omega}=\sup \{u \in P S H(X, \omega) ; u=0 \text { on } E\} .
$$

Then

(a) $\psi_{E, \omega} \in P S H(X, \omega) \cap L^{\infty}(X)$

(b) $\psi_{E, \omega}=0$ on $E$

(c) $\left(\omega+\frac{i}{2} \partial \bar{\partial} \psi_{E, \omega}\right)^{n}=0$ on $X \backslash \bar{E}$.

We can now prove Lemma 4 . Let $E^{\prime} \subset E$ be any relatively compact open subset. Then

$$
\begin{aligned}
\frac{1}{\left[\omega_{t}^{n}\right]} \int_{E^{\prime}} \omega_{t}^{n} & =\frac{1}{\left[\omega_{t}^{n}\right]} e^{-\delta \sup _{X} \psi_{E^{\prime}, \omega_{t}}} \int_{E^{\prime}} e^{-\delta\left(\psi_{E^{\prime} \omega_{t}}-\sup _{X} \psi_{E^{\prime}, \omega_{t}}\right)} \omega_{t}^{n} \\
& \leq e^{-\delta \sup _{X} \psi_{E^{\prime}, \omega_{t}} A \int_{X} e^{-\delta\left(\psi_{E^{\prime}, \omega_{t}}-\sup _{X} \psi_{E^{\prime}, \omega_{t}}\right)} \omega_{0}^{n}}
\end{aligned}
$$

where $A$ is an upper bound for $\frac{1}{\left[\omega_{t}^{n}\right]} \frac{\omega_{t}^{n}}{\omega_{0}^{n}}$. Since $\chi \leq A \omega_{0}$ also by assumption, $P S H\left(X, \omega_{t}\right) \subset$ $P S H\left(X,(A+1) \omega_{0}\right)$, and Theorem A.1 implies that the integral on the right hand side is bounded by a constant independent of $t$ and $E^{\prime}$. We can now complete the proof of Lemma 4. First, observe that if $\sup _{X} \psi_{E^{\prime}, \omega_{t}} \leq 1$, then

$$
\left[\omega_{t}^{n}\right]=\int_{\bar{E}^{\prime}}\left(\omega_{t}+\frac{i}{2} \partial \bar{\partial} \psi_{E^{\prime}, \omega_{t}}\right) \leq \operatorname{Cap}_{\omega_{t}}\left(\bar{E}^{\prime}\right) \leq \operatorname{Cap}_{\omega_{t}}(E) \leq \operatorname{Cap}_{\omega_{t}}(X)=\left[\omega_{t}^{n}\right] .
$$

Thus $\frac{\left[\omega_{t}^{n}\right]}{\operatorname{Cap}_{\omega_{t}}(E)}=1$, and a constant $C_{\delta}$ can clearly be chosen so that the desired inequality holds. Next, assume that $\sup _{X} \psi_{E^{\prime}, \omega_{t}}^{n}>1$. We can write

$$
\begin{aligned}
\left(\sup _{X} \psi_{E^{\prime}, \omega_{t}}\right)^{-n} & =\left(\sup _{X} \psi_{E^{\prime}, \omega_{t}}\right)^{-n} \frac{\int_{X}\left(\omega_{t}+\frac{i}{2} \partial \bar{\partial} \psi_{E^{\prime}, \omega_{t}}\right)^{n}}{\left[\omega_{t}^{n}\right]}=\left(\sup _{X} \psi_{E^{\prime}, \omega_{t}}\right)^{-n} \frac{\int_{\bar{E}^{\prime}}\left(\omega_{t}+\frac{i}{2} \partial \bar{\partial} \psi_{E^{\prime}, \omega_{t}}\right)^{n}}{\left[\omega_{t}^{n}\right]} \\
& \leq \frac{\int_{\bar{E}^{\prime}}\left(\omega_{t}+\frac{i}{2} \partial \bar{\partial}\left(\frac{\psi_{E^{\prime}, \omega_{t}}}{\sup _{X} \psi_{E^{\prime}, \omega_{t}}}\right)\right)^{n}}{\left[\omega_{t}^{n}\right]}
\end{aligned}
$$

This last term is bounded by $\left[\omega_{t}^{n}\right]^{-1} \operatorname{Cap}_{\omega_{t}}\left(\bar{E}^{\prime}\right) \leq\left[\omega_{t}^{n}\right]^{-1} \operatorname{Cap}_{\omega_{t}}(E)$. Thus we obtain

$$
\frac{1}{\left[\omega_{t}^{n}\right]} \int_{E^{\prime}} \omega_{t}^{n} \leq \exp \left(-\delta\left(\frac{\left[\omega_{t}^{n}\right]}{\operatorname{Cap}_{\omega_{t}}(E)}\right)^{\frac{1}{n}}\right) .
$$

Taking limits as $E^{\prime}$ increases to $E$ establishes Lemma 4 . The proof of the theorem is complete. 
We observe that a more straightforward adaption of Kolodziej's original argument can be applied to the special case of algebraic manifolds with the background class being big and semi-ample [ZZh].

Finally, we note that all three proofs of $C^{0}$ estimates can be extended to the equation (1.1) on Hermitian manifolds. For the Moser iteration method, this is carried out in [Ch, TW1, TW2]. It is interesting that, in the Kähler case, only one term in the right hand side of (3.2) was needed, while the other terms are also needed in the Hermitian case. The extension of the pluripotential method to the Hermitian case is in [DK], while the extension of the Alexandrov-Bakelman-Pucci method is in [B11a].

\section{$4 \quad$ Stability Estimates}

In this section, we shall establish the stability and uniqueness of the continuous solutions of the complex Monge-Ampère equations due to Kolodiej [K03]. We shall closely follow the arguments in [K05]. Let $(X, \omega)$ be an $n$-dimensional compact Kähler manifold and let

$$
\mathcal{F}_{p, A}=\left\{F \in L^{p}(X) \mid F \geq 0, \quad \int_{X} F^{p} \omega^{n} \leq A, \quad \int_{X} F \omega^{n}=\int_{X} \omega^{n}\right\}
$$

for $p>1$ and $A>0$.

Theorem 6 For any two $F, G \in \mathcal{F}_{p, A}$, let $\varphi$ and $\psi \in P S H(X, \omega) \cap C(X)$ be solutions of the following Monge-Ampère equations

$$
\left(\omega+\frac{i}{2} \partial \bar{\partial} \varphi\right)^{n}=F(z) \omega^{n}, \quad\left(\omega+\frac{i}{2} \partial \bar{\partial} \psi\right)^{n}=G(z) \omega^{n}
$$

normalized by

$$
\sup _{X} \varphi=\sup _{X} \psi=0
$$

Then for any $\varepsilon>0$, there exists $C>0$ depending only on $\varepsilon, p, A$ and $(X, \omega)$, so that

$$
\|\varphi-\psi\|_{L^{\infty}(X)} \leq C\|F-G\|_{L^{1}(X)}^{\frac{1}{n+3+\varepsilon}}
$$

Theorem 6 has been generalized in [DZ] to nonnegative, big and smooth closed $(1,1)$ form $\omega$ if $\omega$ is chosen appropriately. The uniqueness of the solutions $\varphi \in P S H(X, \omega) \cap$ $C^{0}(X), \sup _{X} \varphi=0$, to the Monge-Ampère equation (3.1) for $F \in \mathcal{F}_{p, A}$ for some $p>1$ and $A>0$ follows immediately from the stability theorem.

As before, we write $\omega_{\varphi}=\omega+\frac{i}{2} \partial \bar{\partial} \varphi$ for any $\varphi \in P S H(X, \omega)$. The following lemma is a generalization of Lemma 3 and a detailed proof can be found in [K05]. 
Lemma 6 Let $\varphi$ and $\psi \in P S H(X, \omega) \cap C(X)$ with $0 \leq \varphi \leq C$. Then for any $s>0$,

$$
\operatorname{Cap}_{\omega}(\{\psi+2 s<\varphi\}) \leq\left(\frac{C+1}{s}\right)^{n} \int_{\psi+s<\varphi} \omega_{\psi}^{n} .
$$

The following lemma is well-known for smooth plurisubharmonic functions. The general result can also be found in [K05].

Lemma 7 Let $\mathcal{B}$ be an Euclidean ball in $\mathbf{C}^{n}$ with the standard Euclidean volume form $\Omega$. For any $u \in P S H(\mathcal{B}) \cap C(\overline{\mathcal{B}})$ and any nonnegative $F \in L^{1}(\overline{\mathcal{B}})$ with

$$
\left(\frac{i}{2} \partial \bar{\partial} u\right)^{n} \geq F \Omega, \quad\left(\frac{i}{2} \partial \bar{\partial} v\right)^{n} \geq F \Omega,
$$

we have

$$
\left(\frac{i}{2} \partial \bar{\partial} u\right)^{k} \wedge\left(\frac{i}{2} \partial \bar{\partial} v\right)^{n-k} \geq F \Omega
$$

for all $k=0, \ldots, n$.

By Theorem 5 , there exists $a>0$ depending on $3^{p} A, p$ and $(X, \omega)$ such that for any $F \in \mathcal{F}_{p, 3^{p} A}$, the solution $u \in P S H(X, \omega) \cap L^{\infty}(X)$ of $\left(\omega+\frac{i}{2} \partial \bar{\partial} u\right)^{n}=F \omega^{n}$ satisfies

$$
\sup _{X} u-\inf _{X} u \leq a .
$$

Without loss of generality, we can assume that $\int_{X} F \omega^{n}=\int_{X} G \omega^{n}=\int_{X} \omega^{n}=1$ and

$$
\int_{\psi<\varphi}(F+G) \omega^{n} \leq 1 .
$$

Furthermore, we let $0<t_{0}<(q-1) / 2$, where $q=(3 / 2)^{1 / n}$ and we further assume $t_{0}^{n+3+\varepsilon}<1 / 3$. For any $0<t<t_{0}$, we define for all nonnegative integers $k$,

$$
E_{k}=\{\psi<\varphi-k a t\}
$$

for a fixed $\varepsilon>0$. From now on we assume that for $0<t<t_{0}$,

$$
\|F-G\|_{L^{1}(X)}=t^{n+3+\varepsilon} .
$$

We have immediately

$$
\int_{E_{0}} G \omega^{n}=\frac{1}{2} \int_{E_{0}}((F+G)+(G-F)) \omega^{n} \leq \frac{1}{2}\left(1+\frac{1}{3}\right)=\frac{2}{3} .
$$

Now we define a new function $H$ such that $H=3 G / 2$ on $E_{0}$ and $H=c_{0}$ on the complement of $E_{0}$ so that $\int_{X} H \omega^{n}=1$. Obviously, $c_{0}>0$ and $H \in \mathcal{F}_{p,(3 / 2)^{p} A}$. Then there exists a unique $\rho \in P S H(X, \omega) \cap C(X)$ such that

$$
\omega_{\rho}^{n}=H \omega^{n}, \quad \sup _{X} \rho=0 .
$$


Furthermore,

$$
-a \leq \rho \leq 0
$$

We now define

$$
E=\{\psi<(1-t) \varphi+t \rho-a t\}, \quad S=\left\{F<\left(1-t^{2}\right) G\right\} .
$$

The following lemma can be easily verified.

\section{Lemma 8}

$$
E_{2} \subset E \subset E_{0} .
$$

Lemma 9 On $E_{0} \backslash S$, for $k=0, \ldots, n$, we have

$$
\omega_{\varphi}^{k} \wedge \omega_{\rho}^{n-k} \geq q^{n-k}\left(1-t^{2}\right)^{k / n} G \omega^{n} .
$$

Proof. On $E_{0} \backslash S$, we have

$$
\left(\left(1-t^{2}\right)^{-1 / n} \omega_{\varphi}\right)^{n} \geq G \omega^{n}, \quad\left(q^{-1} \omega_{\rho}\right)^{n}=G \omega^{n} .
$$

The lemma then follows from Lemma 7. Q.E.D.

Lemma 10 Let $B=\int_{E_{2}} G \omega^{n}$. Then

$$
B \leq \frac{3}{q-1} t^{n+\varepsilon} .
$$

Proof. On $E_{0} \backslash S$, we have

$$
\begin{aligned}
\omega_{(1-t) \varphi+t \rho}^{n} & =\sum_{k=0}^{n}\left(\begin{array}{c}
n \\
k
\end{array}\right)(1-t)^{k} t^{n-k} \omega_{\varphi}^{k} \wedge \omega_{\rho}^{n-k} \\
& \geq \sum_{k=0}^{n}\left(\begin{array}{c}
n \\
k
\end{array}\right) q^{n-k}\left(1-t^{2}\right)^{k / n}(1-t)^{k} t^{n-k} G \omega^{n} .
\end{aligned}
$$

The right hand side can in turn be estimated by

$$
\begin{aligned}
& \sum_{n=0}^{\nu}\left(\begin{array}{l}
n \\
k
\end{array}\right) q^{n-k}\left(1-t^{2}\right) \frac{k}{n}(1-t)^{k} t^{n-k} G \omega^{n}=\left(q t+(1-t)\left(1-t^{2}\right)^{1 / n}\right)^{n} G \\
& \geq\left((1-t)\left(1-t^{2}\right)+q t\right)^{n} G \omega^{n} \geq\left(1+(q-1) t-t^{2}\right)^{n} G \omega^{n} \geq(1+(q-1) t / 2) G \omega^{n},
\end{aligned}
$$

where we make use of the additional assumption that $t<t_{0}<(q-1) / 2$. On the other hand, since $\int_{S} F \omega^{n} \leq\left(1-t^{2}\right) \int_{S} G \omega^{n}$ by the definition of $S$, we have

$$
t^{2} \int_{S} G \omega^{n} \leq \int_{S}(G-F) \omega^{n} \leq t^{n+3+\varepsilon}
$$


and so

$$
\int_{S} G \omega^{n} \leq t^{n+1+\varepsilon}
$$

The above inequality implies that

$$
\int_{E \backslash S} G \omega^{n} \leq \frac{2}{q-1} t^{n+\varepsilon} .
$$

Thus by (4.21) and (4.23),

$$
B \leq \int_{E} G \omega^{n} \leq \int_{E \backslash S} G \omega^{n}+\int_{S} G \omega^{n} \leq \frac{3}{q-1} t^{n+\varepsilon} .
$$

The lemma is then proved. Q.E.D.

\section{Lemma 11}

$$
\operatorname{Cap}_{\omega}\left(E_{4}\right) \leq \frac{3}{q-1}(2 a)^{-n}(a+1)^{n} t^{\epsilon}
$$

Proof. By Lemma 6, we have

$$
\operatorname{Cap}_{\omega}\left(E_{4}\right) \leq \frac{(a+1)^{n}}{(2 a t)^{n}} \int_{E_{2}} G \omega^{n}=\frac{(a+1)^{n}}{(2 a t)^{n}} B \leq \frac{(a+1)^{n}}{(2 a)^{n}} \frac{3}{q-1} t^{\varepsilon} .
$$

The following lemma is used in Kolodziej's original proof of the $L^{\infty}$ estimates. We refer the readers to the detailed proof in [K05].

Lemma 12 Let $\varphi, \psi \in P S H(X, \omega) \cap C(X)$ with $0 \leq \varphi \leq C$. Let

$$
U(s)=\{\psi-s<\varphi\}, \quad \alpha(s)=\operatorname{Cap}_{\omega}(U(s)) .
$$

Assume that

(1) $\{\psi-S<\varphi\} \neq \emptyset$ for some $S$,

(2) For any Borel set $K$,

$$
\int_{K} \omega_{\psi}^{n} \leq f\left(\operatorname{Cap}_{\omega}(K)\right)
$$

where $f(x)=\frac{x}{h\left(x^{-1 / n}\right)}$ and $h(x): \mathbf{R}^{+} \rightarrow(0, \infty)$ is a continuous strictly increasing function satisfying $\int_{1}^{\infty} \frac{1}{t h^{1 / n}(t)} d t<\infty$.

Then for any $D<1$, we have

$$
D \leq \kappa(\alpha(S+D))
$$

where

$$
\kappa(s)=c(n)(1+C)\left(\int_{s^{-1 / n}}^{\infty} \frac{d x}{x h^{1 / n}(x)}+\frac{1}{h^{1 / n}\left(s^{-1 / n}\right)}\right)
$$

for some constant $c(n)$ depending only on $n$. 
Proof of Theorem 6. By Lemma 4, for any $\delta>0$ and open set $K$, there exists $C_{\delta}>0$,

$$
\int_{K} \omega^{n} \leq C_{1} e^{-\left(C_{2} \operatorname{Cap}_{\omega}(K)\right)^{-1}} \leq C_{\delta}\left(\operatorname{Cap}_{\omega}(K)\right)^{1 / \delta} .
$$

Then $0 \leq \varphi+a \leq a$. We can easily check that we can choose $h(x)=x^{1 / \delta}$ and there exists $C_{\delta}^{\prime}>0$ such that

$$
\kappa(s)=C_{\delta}^{\prime} s^{1 /\left(\delta n^{2}\right)} .
$$

Now we can prove the theorem by contradiction. Suppose that

$$
\{\psi<\varphi-(4 a+1) t\}=\{\psi+a<\varphi+a-(4 a+1) t\} \neq \emptyset .
$$

Then by applying Lemma 12 with $\psi+a, \varphi+a, S=-(4 a+1) t$ and $D=t$,

$$
t \leq \kappa\left(\operatorname{Cap}_{\omega}\left(E_{4}\right)\right) \leq \kappa\left(\frac{3}{q-1}(2 a)^{-n}(a+1)^{n} t^{\epsilon}\right)=C_{\delta}^{\prime}\left(\frac{3}{q-1}(2 a)^{-n}(a+1)^{n} t^{\epsilon}\right)^{1 /\left(\delta n^{2}\right)} .
$$

This is a contradiction if we choose $\delta>0$ sufficiently small and then $t>0$ sufficiently small. Therefore $\{\psi<\varphi-(4 a+1) t\}=\emptyset$ and so

$$
\sup _{X}(\varphi-\psi)=\sup _{X}(\psi-\varphi) \leq(4 a+1) t=(4 a+1)\left(\|F-G\|_{L^{1}(X)}\right)^{1 /(n+3+\varepsilon)}
$$

if we choose $t_{0}$ sufficiently small. The theorem is proved. Q.E.D.

\section{A Priori Estimates: $C^{1}$ Estimates}

In Yau's original solution of the Calabi conjecture [Y78], the $C^{2}$ estimates were shown to follow directly from the $C^{0}$ estimates. The $C^{1}$ estimates follow from the $C^{0}$ and $C^{2}$ estimates by general linear elliptic theory. However, for more general Monge-Ampère equations where the right hand side may be an expression $F(z, \varphi)$ depending on the unknown $\varphi$ as well as for the Dirichlet problem, the $C^{1}$ estimates cannot be bypassed. In this section, we describe the sharpest $C^{1}$ estimates available at this time. They are due to [PS09a, PS09c], and they exploit a key differential inequality discovered by Blocki [B09a].

Let $\left(X, \omega_{0}\right)$ be a compact Kähler manifold with smooth boundary $\partial X$ (which may be empty) and complex dimension $n$. We consider the Monge-Ampère equation on $\bar{X}$

$$
\left(\omega_{0}+\frac{i}{2} \partial \bar{\partial} \varphi\right)^{n}=F(z, \varphi) \omega_{0}^{n} .
$$

Here $F(z, \varphi)$ is a $C^{2}$ function on $\bar{X} \times \mathbf{R}$ which is assumed to be strictly positive on the set $\bar{X} \times[\inf \varphi, \infty)$. The gradient estimates allow $\varphi$ to be singular along a subset $Z \subset X$, possibly empty, which does not intersect $\partial X$. All covariant derivatives and curvatures listed below are with respect to the metric $\omega_{0}$. Then [PS09c] 
Theorem 7 Let $\left(X, \omega_{0}\right)$ be a compact Kähler manifold, with smooth boundary $\partial X$ (possibly empty). Assume that $\varphi \in C^{4}(\bar{X} \backslash Z)$ is a solution of the equation (5.1) on $\bar{X} \backslash Z$. If $Z$ is not empty, assume further that $Z$ does not intersect $\partial X$, and that there exists a constant $B>0$ so that

$$
\begin{aligned}
& \varphi(z) \rightarrow+\infty \text { as } z \rightarrow Z, \\
& \log |\nabla \varphi(z)|^{2}-B \varphi(z) \rightarrow-\infty \text { as } z \rightarrow Z
\end{aligned}
$$

Then we have the a priori estimate

$$
|\nabla \varphi(z)|^{2} \leq C_{1} \exp \left(A_{1} \varphi(z)\right), \quad z \in \bar{X} \backslash Z
$$

where $C_{1}$ and $A_{1}$ are constants that depend only on upper bounds for $\inf _{X} \varphi, \sup _{X \times[\inf \varphi, \infty)} F$, $\sup _{X \times[\inf \varphi, \infty)}\left(\left|\nabla F^{\frac{1}{n}}\right|+\left|\partial_{\varphi} F^{\frac{1}{n}}\right|\right), \sup _{\partial X}|\varphi|, \sup _{\partial X}|\nabla \varphi|$, and the following constant,

$$
\Lambda=-\inf _{X} \inf _{M>0} \frac{M^{j}{ }_{k} R^{k}{ }_{j}{ }_{q}\left(M^{-1}\right)^{q} p}{\operatorname{Tr} M \operatorname{Tr} M^{-1}}
$$

where $M=\left(M_{p}^{q}\right)$ runs over all self-adjoint and positive definite endomorphisms.

When there is no boundary, and the function $F(z, \varphi)$ is a function $F(z)$ of $z$ alone, the equation (5.1) is unchanged under shifts of $\varphi$ by an additive constant. Thus the infimum of $\varphi(z)$ can be normalized to be 0 by replacing $\varphi(z) \rightarrow \varphi(z)-\inf _{X} \varphi$, so we obtain the estimate

$$
|\nabla \varphi(z)|^{2} \leq C_{1} \exp \left(A_{1}\left(\varphi(z)-\inf _{X} \varphi\right)\right) \quad z \in \bar{X}
$$

where the constant $C_{1}$ does not depend on $\inf \varphi$, but depends only on the other quantities listed above. We shall see that the Laplacian $\Delta \varphi$ satisfies the same pointwise estimate.

Not surprisingly, the constants $\sup _{M \times[\inf \varphi, \infty]} F$ and $\sup _{M \times[\inf \varphi, \infty]}\left|\nabla F^{\frac{1}{n}}\right|+\left|\partial_{\varphi} F^{\frac{1}{n}}\right|$ in (5.3) can be replaced by $\sup _{M \times[\inf \varphi, \sup \varphi]} F$ and $\sup _{M \times[\inf \varphi, \sup \varphi]}\left|\nabla F^{\frac{1}{n}}\right|+\left|\partial_{\varphi} F^{\frac{1}{n}}\right|$ respectively. Thus, when $\|\varphi\|_{C^{0}}$ is bounded, we obtain gradient bounds for $\varphi$ for completely general smooth and strictly positive functions $F(z, \varphi)$. We have however stated them in the above form since we are particularly interested in the cases when there is no upper bound for $\sup \varphi$. This is crucial for certain applications [PS09a, PS09b]. If a dependence on $\|\varphi\|_{C^{0}}$ is allowed, then there are many earlier direct approaches. The first appears to be due to Hanani [Ha]. More recently, Blocki [B09a] gave a different proof, and our approach builds directly on his. The method of P. Guan [Gp] can be extended to Hessian equations, while the method of B. Guan-Q. Li [GL] allows a general Hermitian metric $\omega$ as well as a more general right hand side $F(z) \chi^{n}$, where $\chi$ is a Kähler form.

The proof is an application of the maximum principle. Let $g_{\bar{k} j}$ and $g_{\bar{k} j}^{\prime}$ be the two metrics defined by the Kähler forms $\omega_{0}$ and $\omega_{0}+\frac{i}{2} \partial \bar{\partial} \varphi$. The covariant derivatives and 
Laplacians with respect to $g_{\bar{k} j}$ and $g_{\bar{k} j}^{\prime}$ are denoted by $\nabla, \Delta$, and $\nabla^{\prime}, \Delta^{\prime}$ respectively. A subindex $g$ or $g^{\prime}$ will denote the metric with respect to which a norm is taken. It is convenient to introduce the endomorphisms

$$
h^{j}{ }_{k}=g^{j \bar{p}} g_{\bar{p} k}^{\prime}, \quad\left(h^{-1}\right)^{j}{ }_{k}=\left(g^{\prime}\right)^{j \bar{p}} g_{\bar{p} k} .
$$

Their traces are $\operatorname{Tr} h=n+\Delta \varphi$ and $\operatorname{Tr} h^{-1}=n+\Delta^{\prime} \varphi$.

As a preliminary, we calculate $\Delta^{\prime} \log |\nabla \varphi|_{g}^{2},|\nabla \varphi|_{g}^{2}$ being the expression of interest, and $\Delta^{\prime}$ being the natural Laplacian to use, as it arises from differentiating the Monge-Ampère equation. We have

$$
\Delta^{\prime} \log |\nabla \varphi|_{g}^{2}=\frac{\Delta^{\prime}|\nabla \varphi|_{g}^{2}}{|\nabla \varphi|_{g}^{2}}-\frac{\left.\left.|\nabla| \nabla \varphi\right|_{g} ^{2}\right|_{g^{\prime}} ^{2}}{|\nabla \varphi|_{g}^{4}}
$$

If we express $\Delta^{\prime}$ on scalars as $\Delta^{\prime}=\left(g^{\prime}\right)^{p \bar{q}} \nabla_{p} \nabla_{\bar{q}}$, then we can write

$$
\Delta^{\prime}|\nabla \varphi|_{g}^{2}=\Delta^{\prime}\left(\nabla_{m} \varphi\right) \nabla^{m} \varphi+\nabla_{m} \varphi \Delta^{\prime}\left(\nabla^{m} \varphi\right)+|\nabla \nabla \varphi|_{g g^{\prime}}^{2}+|\bar{\nabla} \nabla \varphi|_{g g^{\prime}}^{2} .
$$

However, making use of the Monge-Ampère equation, we obtain

$$
\Delta^{\prime}\left(\nabla_{m} \varphi\right)=\left(g^{\prime}\right)^{p \bar{q}} \nabla_{p} \nabla_{\bar{q}} \nabla_{m} \varphi=\left(g^{\prime}\right)^{p \bar{q}} \nabla_{m}\left(\nabla_{p} \nabla_{\bar{q}} \varphi\right)=\partial_{m} \log \frac{\left(\omega^{\prime}\right)^{n}}{\omega^{n}}=\partial_{m} \log F,
$$

while

$$
\Delta^{\prime}\left(\nabla^{m} \varphi\right)=\left(g^{\prime}\right)^{p \bar{q}} \nabla^{m} \nabla_{\bar{q}} \nabla_{p} \varphi+\left(g^{\prime}\right)^{p \bar{q}} R_{\bar{q} p}{ }^{m} \ell \nabla^{\ell} \varphi=\nabla^{m} \log F+\left(h^{-1}\right)^{p}{ }_{r} R^{r}{ }_{p}{ }^{m} \ell \nabla^{\ell} \varphi .
$$

Thus

$$
\Delta^{\prime} \log |\nabla \varphi|_{g}^{2} \geq \frac{2 \operatorname{Re} \nabla_{m} \log F \nabla^{m} \varphi}{|\nabla \varphi|_{g}^{2}}-\Lambda \operatorname{Tr} h^{-1}+\frac{|\nabla \nabla \varphi|_{g g^{\prime}}^{2}+|\bar{\nabla} \nabla \varphi|_{g g^{\prime}}^{2}}{|\nabla \varphi|_{g}^{2}}-\frac{\left.\left.|\nabla| \nabla \varphi\right|_{g} ^{2}\right|_{g^{\prime}} ^{2}}{|\nabla \varphi|_{g}^{4}}
$$

The first term on the right is easily bounded: first write,

$$
\frac{2 \operatorname{Re} \nabla_{m} \log F \nabla^{m} \varphi}{|\nabla \varphi|_{g}^{2}} \geq-2|\nabla \log F|_{g} \frac{1}{|\nabla \varphi|_{g}}=-2 n F^{-\frac{1}{n}}\left|\nabla F^{\frac{1}{n}}\right|_{g} \frac{1}{|\nabla \varphi|_{g}},
$$

and note that

$$
\left|\nabla F^{\frac{1}{n}}\right|_{g} \leq \sup _{X \times[0, \infty)}\left|\partial_{z} F(z, \varphi)^{\frac{1}{n}}\right|+|\nabla \varphi|_{g} \sup _{X \times[0, \infty)}\left|\partial_{\varphi} F(z, \varphi)^{\frac{1}{n}}\right|_{g} \equiv F_{1}^{\prime \prime}+|\nabla \varphi|_{g} F_{1}^{\prime},
$$

while, using the Monge-Ampère equation and the arithmetic-geometric mean inequality,

$$
n F^{-\frac{1}{n}} \leq \operatorname{Tr} h^{-1} .
$$


Thus we find

$$
\Delta^{\prime} \log |\nabla \varphi|_{g}^{2} \geq-\left(\Lambda+2 F_{1}^{\prime}+2 \frac{F_{1}^{\prime \prime}}{|\nabla \varphi|_{g}}\right) \operatorname{Tr} h^{-1}+\frac{|\nabla \nabla \varphi|_{g g^{\prime}}^{2}+|\bar{\nabla} \nabla \varphi|_{g g^{\prime}}^{2}}{|\nabla \varphi|_{g}^{2}}-\frac{\left.\left.|\nabla| \nabla \varphi\right|_{g} ^{2}\right|_{g^{\prime}} ^{2}}{|\nabla \varphi|_{g}^{4}}
$$

The only troublesome term is the negative last term to the right. The key to handling it is a partial cancellation with the two squares preceding it. This cancellation is rather general, and we formalize in the following lemma:

Lemma 13 Let $X$ be a Kähler manifold and $g_{\bar{k} j}, g_{\bar{k} j}^{\prime}$ a pair of Kähler metrics on $M$ (not necessarily in the same Kähler class). Let $\varphi \in C^{\infty}(X)$ and define

$$
S=\langle\nabla \nabla \varphi, \nabla \varphi\rangle_{g}, \quad T=\langle\nabla \varphi, \bar{\nabla} \nabla \varphi\rangle_{g},
$$

Then we have

$$
\frac{|\nabla \nabla \varphi|_{g g^{\prime}}^{2}+|\nabla \bar{\nabla} \varphi|_{g g^{\prime}}^{2}}{|\nabla \varphi|_{g}^{2}} \geq \frac{\left.\left.|\nabla| \nabla \varphi\right|_{g} ^{2}\right|_{g^{\prime}} ^{2}}{|\nabla \varphi|_{g}^{4}}-2 \operatorname{Re}\left\langle\frac{\nabla|\nabla \varphi|_{g}^{2}}{|\nabla \varphi|_{g}^{4}}, T\right\rangle_{g^{\prime}}+2 \frac{|T|_{g^{\prime}}^{2}}{|\nabla \varphi|_{g}^{4}}
$$

Proof of Lemma 13. First, we observe that for all tensors $A_{p i}$ and $B_{j}$ on $X$,

$$
\left|\langle A, B\rangle_{g}\right|_{g^{\prime}}=\left|A_{p i} g^{i \bar{j}} \overline{B_{j}}\right|_{g^{\prime}} \leq|A|_{g g^{\prime}}|B|_{g} .
$$

Now $\nabla|\nabla \varphi|_{g}^{2}=S+T$, and applying (5.17) to $S$ and $T$ gives:

$$
\begin{aligned}
|\nabla \varphi|_{g}^{2} \cdot\left(|\nabla \nabla \varphi|_{g g^{\prime}}^{2}+|\nabla \bar{\nabla} \varphi|_{g g^{\prime}}^{2}\right) & \geq|S|_{g^{\prime}}^{2}+|T|_{g^{\prime}}^{2}=\left.|\nabla| \nabla \varphi\right|_{g} ^{2}-\left.T\right|_{g^{\prime}} ^{2}+|T|_{g^{\prime}}^{2} \\
& =\left.\left.|\nabla| \nabla \varphi\right|_{g} ^{2}\right|_{g^{\prime}} ^{2}-2 \operatorname{Re}\left\langle\nabla|\nabla \varphi|_{g}^{2}, T\right\rangle_{g^{\prime}}+2|T|_{g^{\prime}}^{2}
\end{aligned}
$$

This proves the inequality (5.16).

Returning to the problem of $C^{1}$ estimates, we can now formulate and prove an important inequality due to Blocki at interior critical points of an expression of the form

$$
\log |\nabla \varphi|_{g}^{2}-\gamma(\varphi)
$$

where $\gamma$ is an arbitrary function of a real variable. We apply Lemma 13 as follows: on the right side of (5.16), we drop the third term $2|T|_{g^{\prime}}^{2} /|\nabla \varphi|_{g}^{4}$. In the second term, the tensor $\mathrm{T}$ simplifies upon replacing $\bar{\nabla} \nabla \varphi$ by $g^{\prime}-g$, so that $T$ becomes $T_{j}=\left(\nabla_{i} \varphi\right) g^{i \bar{k}} g_{\bar{k} j}^{\prime}-\nabla_{j} \varphi$. We obtain

$$
\begin{aligned}
\frac{|\nabla \nabla \varphi|_{g g^{\prime}}^{2}+|\nabla \bar{\nabla} \varphi|_{g g^{\prime}}^{2}}{|\nabla \varphi|_{g}^{2}}-\frac{\left.\left.|\nabla| \nabla \varphi\right|_{g} ^{2}\right|_{g^{\prime}} ^{2}}{|\nabla \varphi|_{g}^{4}} & \geq 2 \operatorname{Re}\left\langle\frac{\nabla|\nabla \varphi|_{g}^{2}}{|\nabla \varphi|_{g}^{2}}, \frac{\nabla \varphi}{|\nabla \varphi|_{g}^{2}}\right\rangle_{g^{\prime}}-2 \operatorname{Re}\left\langle\frac{\nabla|\nabla \varphi|_{g}^{2}}{|\nabla \varphi|_{g}^{2}}, \frac{\nabla \varphi}{\left.|\nabla \varphi|_{g}^{2}\right\rangle_{g}}\right. \\
& =2 \gamma^{\prime}(\varphi) \frac{|\nabla \varphi|_{g^{\prime}}^{2}}{|\nabla \varphi|_{g}^{2}}-2 \gamma^{\prime}(\varphi)
\end{aligned}
$$


In the last line, we made use of the fact that $\nabla \log |\nabla \varphi|_{g}^{2}=\gamma^{\prime}(\varphi) \nabla \varphi$ at an interior critical point of the function $\log |\nabla \varphi|_{g}^{2}-\gamma(\varphi)$.

On the other hand,

$$
-\Delta^{\prime} \gamma(\varphi)=-\gamma^{\prime}(\varphi) \Delta^{\prime} \varphi-\gamma^{\prime \prime}(\varphi)|\nabla \varphi|_{g^{\prime}}^{2}=\gamma^{\prime}(\varphi) \operatorname{Tr} h^{-1}-n \gamma^{\prime}(\varphi)-\gamma^{\prime \prime}(\varphi)|\nabla \varphi|_{g^{\prime}}^{2} .
$$

Combining this with the preceding inequality, we obtain Blocki's inequality [B09a],

$$
\begin{aligned}
\Delta^{\prime}\left(\log |\nabla \varphi|_{g}^{2}-\gamma(\varphi)\right) \geq & {\left[\gamma^{\prime}(\varphi)-\Lambda-2 F_{1}^{\prime}-2 \frac{F_{1}^{\prime \prime}}{|\nabla \varphi|_{g}}\right] \operatorname{Tr} h^{-1} } \\
& \quad-(n+2) \gamma^{\prime}(\varphi)-\gamma^{\prime \prime}(\varphi)|\nabla \varphi|_{g^{\prime}}^{2}+2 \gamma^{\prime}(\varphi) \frac{|\nabla \varphi|_{g^{\prime}}^{2}}{|\nabla \varphi|_{g}^{2}}
\end{aligned}
$$

The key to the desired estimate is the following choice of $\gamma(\varphi)$ [PS09a, PS09c]

$$
\gamma(\varphi)=A \varphi-\frac{1}{\varphi+C_{1}}
$$

where $C_{1}$ is chosen to be $C_{1}=-\inf _{X} \varphi+1$, and $A$ is a large positive constant. Then

$$
A \varphi-1 \leq \gamma(\varphi) \leq A \varphi, \quad A \leq \gamma^{\prime}(\varphi) \leq A+1, \quad \gamma^{\prime \prime}(\varphi)=-\frac{2}{\left(\varphi+C_{1}\right)^{3}}<0
$$

and we obtain

$$
\Delta^{\prime}\left(\log |\nabla \varphi|_{g}^{2}-\gamma(\varphi)\right) \geq\left[A-\Lambda-2 F_{1}^{\prime}-2 \frac{F_{1}^{\prime \prime}}{|\nabla \varphi|_{g}}\right] \operatorname{Tr} h^{-1}+\frac{2}{\left(\varphi+C_{1}\right)^{3}}|\nabla \varphi|_{g^{\prime}}^{2}-C_{2}
$$

It suffices to show that, at an interior maximum point $p$, the function $\log |\nabla \varphi|_{g}^{2}-\gamma(\varphi)$ is bounded by an admissible constant. We can assume that $|\nabla \varphi(p)|_{g}^{2} \geq 1$, otherwise the statement follows trivially from the fact that $\gamma(\varphi) \geq A \varphi-1$, and $\varphi$ is bounded from below. Choose $A=\Lambda+2 F_{1}^{\prime}+2 F_{1}^{\prime \prime}+1$. Then the preceding inequality simplifies further to

$$
\Delta^{\prime}\left(\log |\nabla \varphi|_{g}^{2}-\gamma(\varphi)\right) \geq \operatorname{Tr} h^{-1}+\frac{2}{\left(\varphi+C_{1}\right)^{3}}|\nabla \varphi|_{g^{\prime}}^{2}-C_{2}
$$

At an interior minimum point $p$, the left hand side is non-positive. This implies that $\operatorname{Tr} h^{-1}(p)$ is bounded above, and hence the eigenvalues of $h(p)$ are bounded below by a priori constants. In view of the Monge-Ampère equation, they are then bounded above and below by a priori constants, since these constants are allowed to depend on $\sup _{X} F$. This implies that $|\nabla \varphi|_{g^{\prime}}^{2} \geq C_{3}|\nabla \varphi|_{g}^{2}$, and we obtain

$$
|\nabla \varphi|_{g}^{2} \leq C_{4}\left(\varphi+C_{1}\right)^{3}
$$

But we can assume that $\log |\nabla \varphi(p)|_{g}^{2}-\gamma(\varphi(p)) \geq 0$, otherwise there is nothing to prove. Thus $\gamma(\varphi(p)) \leq \log |\nabla \varphi(p)|_{g}^{2}$, and hence

$$
A \varphi(p) \leq \gamma(\varphi(p))+1 \leq \log |\nabla \varphi(p)|_{g}^{2}+1 .
$$


Substituting this in the previous inequality, we find

$$
|\nabla \varphi(p)|_{g}^{2} \leq C_{4}\left(\log |\nabla \varphi(p)|_{g}^{2}+C_{5}\right)^{3} .
$$

This implies that $|\nabla \varphi(p)|_{g}^{2}$ is bounded by an a priori constant. The proof of the $C^{1}$ estimates is complete.

We note that Lemma 13 has other uses. For example, the inequality (5.16) also implies, by completing the square,

$$
\frac{|\nabla \nabla \varphi|_{g g^{\prime}}^{2}+|\nabla \bar{\nabla} \varphi|_{g g^{\prime}}^{2}}{|\nabla \varphi|_{g}^{2}} \geq \frac{1}{2} \frac{\left.\left.|\nabla| \nabla \varphi\right|_{g} ^{2}\right|_{g^{\prime}} ^{2}}{|\nabla \varphi|_{g}^{4}}
$$

This inequality can be used to simplify several estimates in the Kähler-Ricci flow, including the one on the gradient of the Ricci potential.

\section{A Priori Estimates: $C^{2}$ Estimates}

The $C^{2}$ estimates for the complex Monge-Ampère equation (5.1) are due to Yau [Y78] and Aubin $[\mathrm{A}]$. The precise statement is,

Theorem 8 Let $\varphi$ be a $C^{4}$ solution of the equation (2.1) on a compact Kähler manifold $X$, with smooth boundary $\partial X$ (possibly empty). Then

$$
0 \leq n+\Delta \varphi(z) \leq C \exp \left(A_{2}\left(\varphi(z)-\inf _{X} \varphi\right)\right)
$$

where the constant $C$ depends only on an upper bound for $F$, for $\sup _{X \times[\inf \varphi, \infty)}\left|(\log F)_{\varphi}(z, \varphi)\right|$, for the scalar curvature $R$, and a lower bound for $\Delta_{z} \log F$, for $(\log F)_{\varphi \varphi}(z, \varphi)|\nabla \varphi|^{2}$, and for the lower bound $\Lambda$ introduced in (5.4) for the bisectional curvature of $g_{\bar{k} j}$.

When $\partial X$ is not empty, the constant also depends on the boundary value $\varphi_{b}$ of $\varphi$, and on $\|\Delta \varphi\|_{C^{0}(\partial X)}$.

The conclusion still holds for $z \in X \backslash Z$, if the equation (5.1) holds on $X \backslash Z, Z$ is a subset of $X$ not intersecting $\partial X$, and $\varphi(z) \rightarrow+\infty$ as $z \rightarrow Z$.

The derivation of the $C^{2}$ estimates is particularly transparent if we use the formalism of the relative endomorphisms $h$ of (5.6), as in [PSS] and [PS09a], which we follow here. As in the proof of the $C^{1}$ estimates, we would like to estimate $\operatorname{Tr} h$ by the maximum principle. As a preliminary, we calculate

$$
\begin{aligned}
\Delta^{\prime} \operatorname{Tr} h=\left(g^{\prime}\right)^{p \bar{q}} \partial_{\bar{q}} \partial_{p} \operatorname{Tr} h & =\left(g^{\prime}\right)^{p \bar{q}} \operatorname{Tr}\left(\nabla_{\bar{q}}^{\prime}\left(\left(\nabla_{p}^{\prime} h h^{-1}\right) h\right)\right. \\
& =\left(g^{\prime}\right)^{p \bar{q}} \operatorname{Tr}\left(\nabla_{\bar{q}}^{\prime}\left(\nabla_{p}^{\prime} h h^{-1}\right) h\right)+\left(g^{\prime}\right)^{p \bar{q}} \operatorname{Tr}\left(\nabla_{p}^{\prime} h h^{-1} \nabla_{\bar{q}}^{\prime} h\right) .
\end{aligned}
$$

But $\nabla_{\bar{q}}^{\prime}\left(\nabla_{p} h h^{-1}\right)=-R m_{\bar{q} p}^{\prime}+R m_{\bar{q} p}$, as a special case of the general formula comparing the curvatures of two Hermitian metrics on the same holomorphic vector bundle. Here the 
full curvature tensors $R m_{\bar{q} p}$ and $R m_{\bar{q} p}^{\prime}$ are viewed as endomorphisms on the holomorphic tangent bundle. Thus

$$
\begin{aligned}
\left(g^{\prime}\right)^{p \bar{q}} \operatorname{Tr}\left(\nabla_{\bar{q}}^{\prime}\left(\nabla_{p}^{\prime} h h^{-1}\right) h\right) & =-\left(g^{\prime}\right)^{p \bar{q}} R_{\bar{q} p{ }_{k}}^{\prime} h^{k}{ }_{j}+\left(g^{\prime}\right)^{p \bar{q}} R_{\bar{q} p{ }_{k}{ }_{k} h^{k}{ }_{j}} \\
& =-R_{\bar{m} k}^{\prime} g^{k \bar{m}}+\left(h^{-1}\right)^{p}{ }_{m} R^{m}{ }_{p}{ }^{j}{ }_{k} h^{k}{ }_{j} .
\end{aligned}
$$

But the Ricci curvature $R_{\bar{m} k}^{\prime}$ can be obtained from the Monge-Ampère equation

$$
R_{\bar{m} k}^{\prime}=R_{\bar{m} k}-\partial_{k} \partial_{\bar{m}} \log F(z, \varphi) .
$$

Thus we obtain

$$
\Delta^{\prime} \operatorname{Tr} h=-R+\Delta \log F(z, \varphi)+\left(h^{-1}\right)^{p}{ }_{m} R^{m}{ }_{p}^{j}{ }_{k} h_{j}^{k}+\left(g^{\prime}\right)^{p \bar{q}} \operatorname{Tr}\left(\nabla_{p}^{\prime} h h^{-1} \nabla_{\bar{q}}^{\prime} h\right)
$$

and hence

$$
\begin{aligned}
\Delta^{\prime} \log \operatorname{Tr} h= & \frac{-R+\Delta \log F(z, \varphi)+\left(h^{-1}\right)_{m}^{p} R_{p}^{m}{ }_{p}{ }_{k} h^{k}{ }_{j}}{\operatorname{Tr} h} \\
& +\left\{\frac{\left(g^{\prime}\right)^{p \bar{q}} \operatorname{Tr}\left(\nabla_{p}^{\prime} h h^{-1} \nabla_{\bar{q}}^{\prime} h\right)}{\operatorname{Tr} h}-\frac{\left|\nabla^{\prime} \operatorname{Tr} h\right|^{2}}{(\operatorname{Tr} h)^{2}}\right\} .
\end{aligned}
$$

A fundamental inequality due to Yau and Aubin is that the expression between brackets is non-negative, as a consequence of the Cauchy-Schwarz inequality. Also

$$
\Delta(\log F(z, \varphi))=\left(\Delta_{z} \log F\right)(z, \varphi)+(\log F)_{\varphi}(\operatorname{Tr} h-n)+(\log F)_{\varphi \varphi}|\nabla \varphi|^{2} .
$$

Thus

$$
\Delta^{\prime} \log \operatorname{Tr} h \geq-C_{1}(\operatorname{Tr} h)^{-1}-\Lambda \operatorname{Tr} h^{-1}-C_{2} \geq-\left(C_{1}+\Lambda\right) \operatorname{Tr} h^{-1}-C_{2}
$$

since $(\operatorname{Tr} h)^{-1} \leq \operatorname{Tr} h^{-1}$. Here $C_{1}, C_{2}$ depend only on an upper bound for the scalar curvature $R$, a lower bound for $\left(\Delta_{z} \log F\right)(z, \varphi)$, a lower bound for $(\log F)_{\varphi \varphi}|\nabla \varphi|^{2}$, and an upper bound for $\left|(\log F)_{\varphi}\right|$. We can write now

$$
\Delta^{\prime}\left(\log \operatorname{Tr} h-A_{2} \varphi\right) \geq A_{2}\left(\operatorname{Tr} h^{-1}-n\right)-\left(C_{1}+\Lambda\right) \operatorname{Tr} h^{-1}-C_{2} \geq \frac{1}{2} A_{2} \operatorname{Tr} h^{-1}-C_{3}
$$

for $A_{2} \geq 2\left(C_{1}+\Lambda\right)$ and $C_{3}=n A_{2}$. At a maximum point $z_{0}$ for $\log \operatorname{Tr} h-A_{2} \varphi$, the eigenvalues of $h^{-1}$ are then bounded from above by absolute constants. Equivalently, the eigenvalues $\lambda_{i}$ of $h$ are bounded from below by absolute constants, and hence, in view of the Monge-Ampère equation $\prod_{i=1}^{n} \lambda_{i}=F$, they are also bounded from above by constants depending also on $\sup _{X} F$. Thus for any $z \in X$,

$$
\log \operatorname{Tr} h(z) \leq \log \operatorname{Tr} h\left(z_{0}\right)+A_{2}\left(\varphi(z)-\varphi\left(z_{0}\right)\right) .
$$

This establishes the desired $C^{2}$ estimates. We note that the $C^{0}$ bounds for $\Delta \varphi$ imply similar $C^{0}$ bounds for $\partial_{j} \partial_{\bar{k}} \varphi$ by plurisubharmonicity, but not for $\nabla_{j} \nabla_{k} \varphi$. 


\section{A Priori Estimates: the Calabi identity}

To obtain estimates for derivatives of order higher than 2 , we need the equation to be non-degenerate. Thus we allow constants to depend now on a lower bound for $F$. In particular, the $C^{2}$ estimates imply that the metrics $g_{\bar{k} j}$ and $g_{\bar{k} j}^{\prime}$ are equivalent, up to such constants. We restrict ourselves to the equation (3.1), although the arguments can be extended to certain classes of more general $F(z, \varphi)$, for example $F(z, \varphi)=e^{f(z) \pm \varphi}$.

In Yau's solution of the Calabi conjecture [Y78], uniform bounds for the third order derivatives $\nabla_{j} \nabla_{\bar{k}} \nabla_{m} \varphi$ were derived from a generalization to the complex case of an identity due to Calabi [Ca2]. We present here a simplified proof of this identity which appeared in [PSS], and which is again based on the formalism of the relative endomorphism $h^{j}{ }_{k}=$ $g^{j \bar{p}} g_{\bar{p} k}^{\prime}$. Norms and lowering and raising of indices are with respect to $g_{\bar{k} j}^{\prime}$. Covariant derivatives with respect to $g_{\bar{k} j}$ and $g_{\bar{k} j}^{\prime}$ are denoted by $\nabla$ and $\nabla^{\prime}$ respectively.

Define $S=|\nabla \bar{\nabla} \nabla \varphi|^{2}$ as in [Y78]. In terms of $h_{\beta}^{\alpha}$, we have $\left(g^{\prime}\right)^{\alpha \bar{k}} \nabla_{j} \varphi_{\bar{k} \beta}=\left(\nabla_{j}^{\prime} h h^{-1}\right)^{\alpha}{ }_{\beta}$, and thus [PSS]

$$
S=\left|\nabla^{\prime} h h^{-1}\right|^{2}
$$

The point is that the Laplacian of $S$ can now be evaluated directly in terms of metrics and curvatures, instead of Kähler potentials. We readily find

$$
\begin{aligned}
\Delta^{\prime} S= & \left(g^{\prime}\right)^{m \bar{\gamma}}\left(\Delta^{\prime}\left(\nabla_{m}^{\prime} h h^{-1}\right)^{\beta}{ }_{l} \overline{\left(\nabla_{\gamma}^{\prime} h h^{-1}\right)_{\bar{\beta}}^{\bar{\ell}}}+\left(\nabla_{m}^{\prime} h h^{-1}\right)^{\beta}{\overline{\bar{\Delta}^{\prime}}}\left(\nabla_{\gamma}^{\prime} h h^{-1}\right)_{\bar{\beta}}^{\bar{\ell}}\right) \\
& +\left|\bar{\nabla}^{\prime}\left(\nabla^{\prime} h h^{-1}\right)\right|^{2}+\left|\nabla^{\prime}\left(\nabla^{\prime} h h^{-1}\right)\right|^{2}
\end{aligned}
$$

where $\left|\bar{\nabla}^{\prime}\left(\nabla^{\prime} h h^{-1}\right)\right|^{2} \equiv\left(g^{\prime}\right)^{q \bar{p}} \nabla_{\bar{p}}^{\prime}\left(\nabla_{j}^{\prime} h h^{-1}\right)^{\alpha}{ }_{\beta} \overline{\nabla_{\bar{q}}^{\prime}\left(\nabla_{m}^{\prime} h h^{-1}\right)_{\bar{\alpha}}^{\bar{\beta}}}$, and $\Delta^{\prime}=\left(g^{\prime}\right)^{q \bar{p}} \nabla_{q}^{\prime} \nabla_{\bar{p}}^{\prime}, \bar{\Delta}^{\prime}=$ $\left(g^{\prime}\right)^{q \bar{p}} \nabla_{\bar{p}}^{\prime} \nabla_{q}^{\prime}$. Commuting the $\nabla_{q}^{\prime}$ and the $\nabla_{\bar{p}}^{\prime}$ derivatives gives,

$$
\begin{aligned}
\left(\bar{\Delta}^{\prime}\left(\nabla_{j}^{\prime} h h^{-1}\right)\right)_{\alpha}^{\gamma}= & \left(\Delta^{\prime}\left(\nabla_{j}^{\prime} h h^{-1}\right)\right)_{\alpha}^{\gamma}-\left(R^{\prime}\right)^{\gamma}{ }_{\mu}\left(\nabla_{\gamma}^{\prime} h h^{-1}\right)_{\alpha}^{\mu}+\left(R^{\prime}\right)^{\mu}{ }_{\alpha}\left(\nabla_{j}^{\prime} h h^{-1}\right)_{\mu}^{\gamma} \\
& +\left(R^{\prime}\right)^{\mu}{ }_{j}\left(\nabla_{\mu}^{\prime} h h^{-1}\right)_{\alpha}^{\gamma}
\end{aligned}
$$

while, in view of the Bianchi identity,

$$
\begin{aligned}
\Delta^{\prime}\left(\nabla_{j}^{\prime} h h^{-1}\right)_{m}^{l} & =\left(\nabla^{\prime}\right)^{\bar{p}} \partial_{\bar{p}}\left(\nabla_{j}^{\prime} h h^{-1}\right)=-\left(\nabla^{\prime}\right)^{\bar{p}} R_{\bar{p} j m}^{\prime l}+\left(\nabla^{\prime}\right)^{\bar{p}} R_{\bar{p} j m}^{l} \\
& =-\nabla_{j}^{\prime}\left(R^{\prime}\right)_{m}^{l}+\left(\nabla^{\prime}\right)^{\bar{p}} R_{\bar{p} j m}^{l} .
\end{aligned}
$$

with $R_{\bar{p} j m}^{l}=-\partial_{\bar{p}}\left(g^{l}{ }^{l} \partial_{j} g_{\bar{q} m}\right)$. Thus we obtain the exact formula

$$
\begin{aligned}
& \Delta^{\prime} S=\left|\bar{\nabla}^{\prime}\left(\nabla^{\prime} h h^{-1}\right)\right|^{2}+\left|\nabla^{\prime}\left(\nabla^{\prime} h h^{-1}\right)\right|^{2} \\
& -\left(\left(\nabla^{\prime}\right)^{\bar{\gamma}} R_{\bar{\beta} \alpha}^{\prime} \overline{\left(\nabla_{\gamma}^{\prime} h h^{-1}\right)^{\beta \bar{\alpha}}}+\left(\left(\nabla^{\prime}\right)^{\bar{\gamma}} h h^{-1}\right)^{\beta \bar{\alpha}} \overline{\nabla_{\gamma}^{\prime} R_{\bar{\beta} \ell}^{\prime}}\right) \\
& +\left(\nabla_{m}^{\prime} h h^{-1}\right)^{\beta}{ }_{l}\left(\left(R^{\prime}\right)^{l \bar{\rho}} \overline{\left(\left(\nabla^{\prime}\right)^{\bar{m}} h h^{-1}\right)_{\bar{\beta} \rho}}-R_{\bar{\rho} \beta}^{\prime} \overline{\left(\left(\nabla^{\prime}\right)^{\bar{m}} h h^{-1}\right)^{\rho \bar{l}}}+\left(R^{\prime}\right)^{m \bar{\rho}} \overline{\left(\nabla_{\rho}^{\prime} h h^{-1}\right)_{\bar{\beta}}^{\bar{l}}}\right)
\end{aligned}
$$

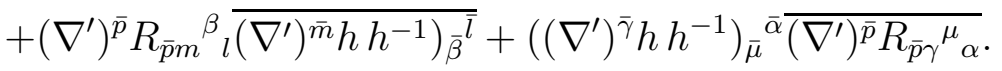


Since $R_{\bar{p} m}^{\prime}=R_{\bar{p} m}-\partial_{m} \partial_{\bar{p}} \log F$, it can be viewed as known. As already noted, the metrics $g_{\bar{k} j}$ and $g_{\bar{k} j}^{\prime}$ are uniformly equivalent. Since the connection $\nabla^{\prime} h h^{-1}$ is of order $O\left(S^{\frac{1}{2}}\right)$, the above identity implies

$$
\Delta^{\prime} S \geq-C_{1} S-C_{2}
$$

where the constants $C_{i}$ depend on an upper bound for $\Delta \varphi$, a lower bound for $F$, the $C^{3}$ norm of $F$, and the $C^{1}$ norms of the curvature $R_{\bar{k} j m}{ }^{l}$ of $g_{\bar{k} j}$. Using the expression (6.5) for $\Delta^{\prime} \operatorname{Tr} h$, and the fact that

$$
\left(g^{\prime}\right)^{p \bar{q}} \operatorname{Tr}\left(\nabla_{p}^{\prime} h h^{-1} \nabla_{\bar{q}}^{\prime} h\right)=\left(g^{\prime}\right)^{p \bar{q}}\left(g^{\prime}\right)^{j \bar{k}} g^{m \bar{r}} \nabla_{p} \nabla_{\bar{m}} \nabla_{j} \varphi \overline{\nabla_{q} \nabla_{\bar{m}} \nabla_{k} \varphi} \geq C_{3} S
$$

since the metrics $g_{\bar{k} j}$ and $g_{\bar{k} j}^{\prime}$ are equivalent, we readily see that

$$
\Delta^{\prime}(S+A \operatorname{Tr} h) \geq C_{4} S-C_{5}
$$

for $A$ sufficiently large. We can now apply the maximum principle and obtain the following:

Theorem 9 Let $\varphi$ be a $C^{5}$ solution of the equation (5.1) on a compact Kähler manifold $\left(X, \omega_{0}\right)$ with smooth boundary $\partial X$ (possibly empty). Then that $S$ is uniformly bounded by constants depending only on the $C^{0}$ norms of $\partial_{j} \partial_{\bar{k}} \log F$ and $\nabla_{j} \nabla_{\bar{k}} \nabla_{m} \log F$, a lower bound for $F$, the $C^{1}$ norm of $R_{\bar{k}_{j}}{ }^{l}$, and, when $\partial X$ is not empty, on $\|S\|_{C^{0}(\partial X)}$.

We conclude this section by noting that most of the a priori estimates discussed here have counterparts for parabolic Monge-Ampère equations. They were instrumental in Cao's proof of the all-time existence for the Kähler-Ricci flow on manifolds of definite Chern classes [Cao]. They also apply in many situations to manifolds of general type [Ts, EGZ, ST09]. For the modified Kähler-Ricci flow, the $C^{3}$ estimates and the Calabi identity require an additional argument [PSSW2], as well as a full use of the square terms in (7.4) which were dropped in the proof of Theorem 9. Extensions to flows on Hermitian manifolds can be found in [Gm, ZZ].

\section{Boundary Regularity}

In this section, we discuss a priori estimates for the Dirichlet problem for the complex Monge-Ampère equation on a Kähler manifold $\left(X, \omega_{0}\right)$ with smooth boundary $\partial X$.

\section{1 $C^{0}$ estimates}

Let $\varphi_{b}$ be a smooth function on $\partial X$, and consider the Dirichlet problem

$$
\left(\omega_{0}+\frac{i}{2} \partial \bar{\partial} \varphi\right)^{n}=F(z, \varphi, \nabla \varphi) \omega_{0}^{n} \text { on } X, \quad \varphi=\varphi_{b} \text { on } \partial X
$$


where $n=\operatorname{dim} X, F$ is a smooth strictly positive function, and $\varphi \in P S H\left(X, \omega_{0}\right) \cap C^{\infty}(X)$.

The fact that $\varphi \in P S H\left(X, \omega_{0}\right)$ implies that $n+\Delta \varphi \geq 0$. If $h$ is the solution of the Dirichlet problem $\Delta h=-n$ on $X, h=\varphi_{b}$ on $\partial X$, then the comparison principle implies

$$
\varphi \leq h .
$$

Thus, to obtain $C^{0}$ estimates, we need only a lower bound for $\varphi$. As shown by Caffarelli, Kohn, Nirenberg, and Spruck [CNS, CKNS], this can be effectively obtained if we assume the existence of a smooth subsolution $\underline{\varphi}$ of the Dirichlet problem (9.1), that is, a smooth function $\underline{\varphi}$ satisfying

$$
\left(\omega_{0}+\frac{i}{2} \partial \bar{\partial} \underline{\varphi}\right)^{n}>F(z, \underline{\varphi}, \nabla \underline{\varphi}) \omega_{0}^{n} \text { on } X, \quad \underline{\varphi}=\varphi_{b} \text { on } \partial X .
$$

Indeed, in the method of continuity, the problem reduces to a priori estimates for the equation

$$
\begin{aligned}
\left(\omega_{0}+\frac{i}{2} \partial \bar{\partial} \varphi\right)^{n} & =t F(z, \varphi, \nabla \varphi) \omega_{0}^{n}+(1-t)\left(\omega_{0}+\frac{i}{2} \partial \bar{\partial} \underline{\varphi}\right)^{n} \text { on } X, \\
\varphi & =\varphi_{b} \text { on } \partial X,
\end{aligned}
$$

for $0 \leq t \leq 1$. Let $\varphi=\underline{\varphi}$ for $t=0$. We claim that, if a smooth solution exists in an interval $0 \leq t<T$, then

$$
\underline{\varphi}<\varphi \text { in } X,
$$

for all $t<T$. To see this, note that the derivative in $t$ of $\frac{\left(\omega_{0}+\frac{i}{2} \partial \bar{\partial} \varphi\right)^{n}}{\left(\omega_{0}+\frac{i}{2} \partial \bar{\partial} \varphi\right)^{n}}$ is strictly negative at $t=0$. Thus $\left(\omega_{0}+\frac{i}{2} \partial \bar{\partial} \varphi\right)^{n}<\left(\omega_{0}+\frac{i}{2} \partial \bar{\partial} \underline{\varphi}\right)^{n}$, and $\underline{\varphi}<\varphi$ for $\bar{t}$ strictly positive and small, by the comparison principle. If there exists $t_{0}, 0<t_{0}<T$, with $\underline{\varphi}\left(z_{0}\right)=\varphi\left(z_{0}\right)$ for some $z_{0} \in X$, let $t_{0}$ be the first such time. By continuity, $\underline{\varphi}(z) \leq \varphi(z)$ for all $z \in X$ and $t=t_{0}$, so $z_{0}$ is a maximum of the function $\underline{\varphi}-\varphi$ at $t_{0}$. In particular, at $t_{0}$ and $z_{0}$, we have $\nabla \underline{\varphi}=\nabla \varphi$ and

$$
\left(\omega_{0}+\frac{i}{2} \partial \bar{\partial} \underline{\varphi}\right)^{n} \leq\left(\omega_{0}+\frac{i}{2} \partial \bar{\partial} \varphi\right)^{n} .
$$

But the equation (8.4) implies, again at $t_{0}$ and $z_{0}$,

$$
\left(\omega_{0}+\frac{i}{2} \partial \bar{\partial} \varphi\right)^{n}=t F(z, \underline{\varphi}, \nabla \underline{\varphi})+(1-t)\left(\omega_{0}+\frac{i}{2} \partial \bar{\partial} \underline{\varphi}\right)^{n}<\left(\omega_{0}+\frac{i}{2} \partial \bar{\partial} \underline{\varphi}\right)^{n},
$$

which is a contradiction.

\section{2 $C^{1}$ boundary estimates}

The $C^{1}$ estimates at the boundary $\partial X$ follow from the bounds $\underline{\varphi} \leq u \leq h$, and the fact that all three functions have the same boundary values. When the right hand side $F(z, \varphi, \nabla \varphi)$ does not depend on $\nabla \varphi$, the estimates established earlier in Section 4 show that the interior $C^{1}$ estimates can be reduced to the boundary $C^{1}$ estimates. 


\section{3 $C^{2}$ boundary estimates of Caffarelli-Kohn-Nirenberg-Spruck and B. Guan}

The barrier constructions of Caffarelli, Kohn, Nirenberg, Spruck [CKNS] and B. Guan $[\mathrm{Gb}]$ provide $C^{0}(\partial X)$ bounds for $\Delta \varphi$, in terms of $C^{0}(X)$ bounds for $\varphi$ and for $\nabla \varphi$. The following slightly more precise formulation of their estimates can be found in [PS09a], under the simplifying assumption that the boundary $\partial X$ is holomorphically flat ${ }^{2}$ :

Theorem 10 Assume that $\partial X$ is holomorphically flat, and that $\varphi$ is a $C^{3}$ solution of the equation (8.1), with $F(z)$ on the right-hand side. Then we have

$$
\sup _{\partial X}(n+\Delta \varphi) \leq C \sup _{\partial X}\left(1+|\nabla \varphi|^{2}\right) \sup _{X}\left(1+|\nabla \varphi|^{2}\right),
$$

for a constant $C$ depending only on the boundary $\partial X, \omega_{0}$, and upper bounds for $\sup _{X} F$, and $\sup _{X}(\nabla \log F)$, and $\|\varphi\|_{C^{0}(X)},\|\nabla \varphi\|_{C^{0}(X)}$.

By the interior estimates of Yau and Aubin in $\S 6$, the uniform bound for $\Delta \varphi$ in the whole of $X$ can be reduced to its estimate on $\partial X$. Thus the above bound implies that $\|\Delta \varphi\|_{C^{0}(X)}$ is bounded in terms of the constants indicated. By plurisubharmonicity, it follows that all the mixed partials $\left\|\partial_{j} \partial_{\bar{k}} \varphi\right\|_{C^{0}(X)}$ are bounded as well.

It is an interesting question whether bounds for the un-mixed partials $\left\|\nabla_{j} \nabla_{k} \varphi\right\|_{C^{0}}$ can be obtained as well without additional assumptions. Such bounds have been obtained by Blocki [B09b] under the additional assumption that the background form $\omega_{0}$ has nonnegative bisectional curvature.

If we allow bounds to depend on a lower bound for $F$, then the equation (8.3) can be viewed as uniformly elliptic, since the eigenvalues of the relative endomorphism $h^{j}{ }_{k}=$ $g^{j \bar{p}} g_{\bar{p} k}^{\prime}$ are already known to be bounded from above, and using the lower bound for $F$, they are also bounded from below. The Monge-Ampère equation is concave, so we can then apply to the following general theorem of the Evans-Krylov and Krylov theory, which we quote from Chen-Wu [CW] (see also Gilbarg-Trudinger [GT] p. 482 and Q. Han $[\mathrm{H}]$ ). The statement is local, and can be formulated for domains with smooth boundary in $\mathbf{R}^{n}$ :

Theorem 11 Assume that $\Omega \subset \mathbf{R}^{n}$ has smooth boundary, and the boundary data is smooth. Assume that $F\left(x, u, D u, D^{2} u\right)$ is smooth in all variables $(x, u, p, A)$, uniformly elliptic and concave (or convex) in $D^{2} u$, and assume that $\|u\|_{C^{1, \gamma(\bar{\Omega})}}$ is bounded for some $0<\gamma<1$. Then there are constants $0<\alpha<\gamma$ and $C$ so that, for any $0<\beta<\alpha$, we have

$$
\|u\|_{C^{2, \beta}(\bar{\Omega})} \leq C
$$

\footnotetext{
${ }^{2} \mathrm{~A}$ hypersurface $\partial X$ is holomorphically flat if, locally, there exist holomorphic coordinates $\left(z_{1}, \cdots, z_{n}\right)$ so that $\partial X$ is given by $\operatorname{Re} z_{n}=0$.
} 
We note that, while both the local [Ca] and the global [TrWa] $C^{2, \alpha}$ regularity is known for real Monge-Ampère equations when the right hand side $F$ is in $C^{\alpha}$, the corresponding question is still not completely resolved in the complex case. For some recent progress on this issue, see [DZZ], and particularly [W2], where it is shown that the solution $\varphi$ is of class $C^{2, \alpha}$ if the right hand side $F(z)$ is strictly positive, $F^{\frac{1}{n}} \in C^{\alpha}$, and $\Delta \varphi$ is bounded.

\section{The Dirichlet Problem for the Monge-Ampère equa- tion}

The preceding a priori estimates imply the following classic existence theorem due to Caffarelli, Kohn, Nirenberg, and Spruck [CKNS] and B. Guan [Gb]:

Theorem 12 Let $\left(\bar{X}, \omega_{0}\right)$ be a compact Kähler manifold of dimension n, with smooth boundary $\partial X$. Let $F(z, \varphi)$ be a smooth, strictly positive function of the variables $z$ and $\varphi$, and let $\varphi_{b}$ be a smooth function on $\partial X$. Consider the Dirichlet problem

$$
\left(\omega_{0}+\frac{i}{2} \partial \bar{\partial} \varphi\right)^{n}=F(z, \varphi) \omega_{0}^{n}, \quad \varphi=\varphi_{b} \text { on } \partial X .
$$

If $F_{\varphi}(z, \varphi) \geq 0$ and the problem admits a smooth subsolution, that is, a smooth function $\underline{\varphi}$ satisfying

$$
\left(\omega_{0}+\frac{i}{2} \partial \bar{\partial} \underline{\varphi}\right)^{n}>F(z, \underline{\varphi}) \omega_{0}^{n}, \quad \underline{\varphi}=\varphi_{b} \text { on } \partial X,
$$

then the Dirichlet problem (9.1) admits a unique solution $\varphi$, and $\varphi \in C^{\infty}(\bar{X})$.

Indeed, the $C^{0}$ estimates of $\S 8.1$, the $C^{2}$ estimates of $\S 6$ and $\S 8.2$, the Evans-Krylov theory for higher derivatives of $\S 8.2$ can be applied to show that the equation (8.4) admits a solution for $0 \leq t \leq 1$.

Similar results for the real Monge-Ampère equation can be found in [GS] and [Gb98]. An extension to Hermitian manifolds can be found in [GL].

The a priori estimates show more than just the existence of a solution $\varphi$ for the equation (9.1): the upper bound for $\Delta \varphi$ does not depend on a lower bound for $F(z, \varphi)$. This allows an immediate application to the existence of solutions to the Dirichlet problem for the completely degenerate, or homogeneous, complex Monge-Ampère equation. For this, we apply Theorem 12 to the Dirichlet problem

$$
\left(\omega_{0}+\frac{i}{2} \partial \bar{\partial} \varphi_{\varepsilon}\right)^{n}=\varepsilon \omega_{0}^{n}, \quad \varphi_{\varepsilon}=0 \text { on } \partial X,
$$

where $\varepsilon$ is a constant satisfying $0<\varepsilon<1$. The function $\underline{\varphi}_{\varepsilon}=0$ is a subsolution, and hence Theorem 12 implies the existence of a smooth solution $\varphi_{s}$ with $\Delta \varphi_{s}$ bounded uniformly 
in $\varepsilon$. Thus a subsequence of the functions $\varphi_{\varepsilon}$ converges in $C^{1, \alpha}$ to a $C^{1, \alpha}$ solution of the equation (9.4) for all $0<\alpha<1$. We obtain in this manner the following theorem, whose present formulation is due to Blocki [B09b] and which generalizes the theorem of Chen [C00] stated further below as Theorem 24:

Theorem 13 Let $\left(X, \omega_{0}\right)$ be a compact Kähler manifold with smooth boundary $\partial X$. Then the Dirichlet problem

$$
\left(\omega_{0}+\frac{i}{2} \partial \bar{\partial} \varphi\right)^{n}=0 \text { on } \partial X, \quad \varphi=0 \text { on } \partial X
$$

admits a unique solution, which is of class $C^{1, \alpha}(\bar{X})$ for each $0<\alpha<1$.

In some applications, as in the problem of geodesics in the space of Kähler potentials described below in Section $§ 13$, it is actually necessary to consider equations of the form (9.4), but with the Kähler form $\omega_{0}$ replaced by a smooth background $(1,1)$-form $\omega$ which is closed, non-negative, but not strictly positive. We discuss a specific situation where the existence and regularity of solutions can still be established by the a priori estimates that we described in sections $\S 5, \S 6, \S 8.2$, and $\S 8.3$ (in fact, some of the $C^{1}$ estimates given in $\S 5$ were designed for that purpose).

Assume that $\omega$ is a smooth, closed, and non-negative $(1,1)$-form, and that there exists an effective divisor $E$, not intersecting $\partial X$, with the line bundle $O(E)$ admitting a metric $K$ satisfying

$$
\omega_{K} \equiv \omega+\delta \frac{i}{2} \partial \bar{\partial} \log K>0
$$

for some strictly positive constant $\delta$. Then we have the following theorem [PS09a]:

Theorem 14 Let $X$ be a compact complex manifold with smooth boundary $\partial X$. Assume that $\omega$ is a smooth non-negative $(1,1)$-form, $E$ is an effective divisor not intersecting $\partial X$, $K$ is a metric on $O(E)$, with $\omega_{K}$ satisfying the Kähler condition (9.5). Then the Dirichlet problem

$$
\left(\omega+\frac{i}{2} \partial \bar{\partial} \varphi\right)^{n}=0 \text { on } X, \quad \varphi=0 \text { on } \partial X
$$

admits a unique bounded solution. The solution is $C^{\alpha}(\bar{X} \backslash E)$ for any $0<\alpha<1$. If $\partial X$ is holomorphically flat (in the sense that there exists holomorphic coordinates $z_{i}$ with $\partial X=\left\{\operatorname{Re} z_{n}=0\right\}$ locally), then the solution is $C^{1, \alpha}(\bar{X} \backslash E)$ for any $0<\alpha<1$.

We sketch the proof. Let

$$
\omega_{s}=(1-s) \omega_{0}+s \omega_{K}
$$


For $0<s<1, \omega_{s}$ is strictly positive definite. Consider the equation

$$
\left(\omega_{s}+\frac{i}{2} \partial \bar{\partial} \varphi_{s}\right)^{n}=F_{s}(z) \omega_{s}^{n} \text { on } X, \quad \varphi_{s}=0 \text { on } \partial X
$$

for some smooth functions $F_{s}>0$ satisfying $\sup _{X} F_{s}<1$, to be specified more completely later. By Theorem 12, this equation admits a smooth solution in $P S H\left(X, \omega_{s}\right)$ for each $s>0$. Since the eigenvalues of $\omega_{s}$ are bounded from above with respect to the Kähler form $\omega_{K}$, the $\omega_{s}$-plurisubharmonicity of $\varphi_{s}$ implies that $\Delta_{\omega_{K}} \varphi_{s} \geq-C$ for a uniform constant $C$. The arguments for $C^{0}$ estimates in $\S 8.1$ imply that the norms $\left\|\varphi_{s}\right\|_{C^{0}(X)}$ are uniformly bounded in $s$.

To obtain $C^{1}$ estimates on compact subsets of $X \backslash E$, we choose $F_{s}$ as follows. First, define

$$
\hat{\omega}_{s}=\omega_{K}+s \frac{i}{2} \partial \bar{\partial} \log K^{\delta} .
$$

Then $\hat{\omega}_{s}$ is uniformly bounded from below for all $s$ sufficiently small. In particular, its curvature tensor is uniformly bounded together with all its derivatives. On the other hand, since $\hat{\omega}_{s}$ can also be expressed as

$$
\hat{\omega}_{s}=\omega_{s}+\frac{i}{2} \partial \bar{\partial} \log K^{\delta}
$$

the equation (9.8) can be rewritten as

$$
\left(\hat{\omega}_{s}+\frac{i}{2} \partial \bar{\partial}\left(\varphi_{s}-\delta \log \|\psi\|_{K}^{2}\right)\right)^{n}=\hat{F}_{s} \hat{\omega}_{s}^{n}
$$

with $\psi$ a holomorphic section of $O(E),\|\psi\|_{K}^{2}=\psi \bar{\psi} K$, and $\hat{F}_{s} \hat{\omega}_{s}^{n}=F_{s} \omega_{s}^{n}$. Choose $F_{s}$ to be constants tending so fast to 0 that $\limsup _{s \rightarrow 0}\left\|F_{s}\right\|_{C^{0}(X)}=0$. The desired uniform bounds for $\nabla \varphi_{s}$ on compact subsets of $X \backslash E$ follow from the $C^{1}$ estimates of $\S 5$. With these estimates, it is then easy to show the existence of a subsequence of $\varphi_{s}$ converging in $C^{\alpha}$ on compact subsets of $X \backslash E$ to a solution of (9.6).

\section{Singular Monge-Ampère equations}

In the seminal paper [Y78], Yau not only solved the Calabi conjecture, but he also started the study of complex Monge-Ampère equations in more general settings. These include settings when the right hand side may have zeroes or poles, or when the manifold $X$ is not compact and one looks for a complete Kähler-Einstein metric, or when $X$ is quasiprojective. We recall briefly some of these classical results below, before discussing some more recent developments. In these more recent developments, the underlying manifold may have singularities, and/or the background form $\omega_{0}$ in the Monge-Ampère equation may be degenerate. 


\subsection{Classic works}

The classical literature on singular Monge-Ampère equations and singular Kähler-Einstein metrics is particularly rich, as different equations are required by different geometric situations. We shall restrict ourselves to describing three results.

First, we consider the case when the right hand side of the equation has zeroes and/or poles. Let $\left(X, \omega_{0}\right)$ be a compact Kähler manifold. Let $\left\{L_{i}\right\}_{i=1}^{I}$ be a family of holomorphic line bundles over $X$. For each $i$, let $s_{i}$ be a holomorphic section of $L_{i}$ and $h_{i}$ a smooth hermitian metric on $L_{i}$. Let

$$
u_{k}=\sum_{i=1}^{I} a_{k, i}\left|s_{i}\right|_{h_{i}}^{2 \alpha_{k, i}}, k=1, \ldots, K, \quad v_{l}=\sum_{i=1}^{I} b_{l, i}\left|s_{i}\right|_{h_{i}}^{2 \beta_{l, i}} l=1, \ldots, L,
$$

where $a_{k, i} b_{l, i}, \alpha_{k, i}$ and $\beta_{l, i}$ are nonnegative numbers, and consider the following MongeAmpère equation

$$
\left(\omega_{0}+\frac{i}{2} \partial \bar{\partial} \varphi\right)^{n}=\frac{u_{1} u_{2} \ldots u_{K}}{v_{1} v_{2} \ldots v_{L}} e^{f(z)} \omega_{0}^{n},
$$

where $f=f(z)$ is a smooth function on $X$,. The following theorem is due to Yau [Y78]:

Theorem 15 Assume the following two conditions:

(1) $\left(u_{1} u_{2} \ldots u_{K}\right)\left(v_{1} v_{2} \ldots v_{L}\right)^{-1} e^{f} \in L^{n}(X)$ and

$$
\int_{X} \frac{u_{1} u_{2} \ldots u_{K}}{v_{1} v_{2} \ldots v_{L}} e^{f} \omega_{0}^{n}=\int_{X} \omega_{0}^{n}
$$

(2) there exists $\epsilon>0$ such that

$$
\left(v_{1} v_{2} \ldots v_{L}\right)^{-\epsilon}\left|\Delta \log \left(v_{1} v_{2} \ldots v_{L}\right)\right|^{(n-1) / n} \in L^{1}(X \backslash D)
$$

where $\Delta$ is the Laplacian with respect to $\omega_{0}$ and $D$ is the union of the zeros of $v_{l}, l=1, \ldots, L$.

Then there exists a bounded $\omega_{0}$-psh function $\varphi$ solving the equation (10.2). Furthermore, $\varphi$ is smooth outside the zeros of $u_{k}$ and $v_{l}$, for $k=1, \ldots, K$ and $l=1, \ldots, L$, and $\varphi$ is unique up to a constant.

In particular, if for each $k$ and $l, u_{k}=a_{i_{k}}\left|s_{i_{k}}\right|_{h_{i_{k}}}^{2 \alpha_{i_{k}}}$ and $v_{l}=b_{i_{l}}\left|s_{i_{l}}\right|_{h_{i_{l}}}^{2 \beta_{i_{l}}}$ for some $1 \leq i_{k} \leq K$ and $1 \leq i_{l} \leq I$, the second assumption in Theorem 15 holds automatically. The first assumption for Theorem 15 is that the right hand side of the equation $(10.2)$ is in $L^{n}(X)$. Thus the theorem on $C^{0}$ estimates of Kolodziej can also be applied here, and we can obtain in this manner a new proof of Theorem 15.

The next important geometric situation is that of open complex manifolds. There one is interested in complete Kähler-Einstein metrics of negative curvature. In [CY80], Cheng and Yau gave effective criteria for the existence of such metrics. In particular, they proved the existence of a complete Kähler-Einstein metric of negative scalar curvature on bounded, 
smooth, strictly pseudoconvex domains in $\mathbf{C}^{n}$. This corresponds to solving Monge-Ampère equations of the form (2.1), with the solution tending to $\infty$ at the boundary. This also allowed Cheng and Yau to obtain essentially sharp boundary regularity results for the Dirichlet problem for the closely related equation $J(u)=1$ of Fefferman [F76]. It was subsequently shown by Mok and Yau [MY83] that any bounded domain of holomorphy admits a complete Kähler-Einstein metric.

A third important class of non-compact manifolds is the class of quasi-projective manifolds. Let $M=\bar{M} \backslash D$ be a quasi-projective manifold, where $\bar{M}$ is a projective manifold and $D$ is a smooth ample divisor on $M$. The Calabi conjecture for quasi-projective manifolds asserts that, for any smooth real valued $(1,1)$-form $\eta \in c_{1}\left(K_{\bar{M}}^{-1} \otimes[D]^{-1}\right)$, there exists a complete Kähler metric on $M$ with its Ricci curvature equal to $\left.\eta\right|_{M}$. This was proved by Tian and Yau in [TY86, TY90, TY91].

These works required at that time many new technical tools which remain useful to this day. They include the notion of bounded geometry, the Cheng-Yau Hölder spaces with weights, and particularly the observation repeatedly stressed in these works that the arguments are almost local in nature, and that the manifold can be allowed singularities, as long as the metric admits a non-singular resolution by a local holomorphic map.

\subsection{Monge-Ampère equations on normal projective varieties}

The original theorems of Yau [Y78] and Yau [Y78] and Aubin [A] establish the existence of Kähler-Einstein metrics on a Kähler manifold $X$ when $K_{X}$ has zero or positive first Chern class. We discuss now one of the new developments in the theory of complex MongeAmpère equations, namely an extension of these results to normal projective manifolds. Normal projective manifolds are a very specific class of manifolds with singularities. For the convenience of the reader, we summarize here some of their basic definitions and properties.

Let $X$ be a subvariety of $\mathbf{C P}^{N}$. A function on a neighborhood of a point $z_{0} \in X$ is holomorphic if it extends to a holomorphic function on a neighborhood of $z_{0} \in \mathbf{C P}^{N}$. Let $X_{\text {sing }}$ be the smallest subset of $X$ with $X \backslash X_{\text {sing }}$ a complex manifold. Then $X$ is said to be normal if for any $z_{0} \in X_{\text {sing }}$, there is a neighborhood $U$ of $z_{0}$ so that any bounded holomorphic function on $U \backslash X_{\text {sing }}$ extends to a holomorphic function on $U$.

A plurisubharmonic function on $U \subset X$ is by definition the restriction to $X$ of a plurisubharmonic function in a neighborhood $\hat{U}$ of $U$ in $\mathbf{C P}^{N}$. By a theorem of Fornaess and Narasimhan [FN], if $X$ be a normal projective variety, and a function $\varphi$ is plurisubharmonic on $U \backslash X_{\text {sing }}$ and is bounded, then $\varphi$ is plurisubharmonic on $U$.

A line bundle $L$ on $X$ is an ample Q-line bundle if $m L$ is the restriction to $X$ of $O(1)$ for some $m \in \mathbf{Z}^{+}$. More generally, a line bundle $\tilde{L} \rightarrow \tilde{X}$ is an ample Q-line bundle if there is an imbedding of $\tilde{X}$ into projective space, with the pull-back of $O(1)$ equal to $m \tilde{L}$ for some $m \in \mathbf{Z}^{+}$. 
We can define now the notion of Monge-Ampère measure on a normal projective variety $X$. Let $\operatorname{dim} X=n$, and let $\pi: \tilde{X} \rightarrow X$ be a smooth resolution of singularities of $X$. Let $\tilde{L}=\pi^{*} L$ for any ample Q-line bundle $L \rightarrow X$. By definition, $m L=O(1)$ for some $m \in \mathbf{Z}^{+}$ and so

$$
m \tilde{L}=\pi^{*} O(1)
$$

Let $m \omega$ be the restriction of the Fubini-Study metric on $\mathbf{C P}^{M}$ to $X$ and let $\tilde{\omega}=\pi^{*} \omega$. For any bounded $\omega$-plurisubharmonic function $\varphi$ on $X$, we let $\tilde{\varphi}=\pi^{*} \varphi$. The measure $\left(\tilde{\omega}+\frac{i}{2} \partial \bar{\partial} \tilde{\varphi}\right)^{n}$ is a well-defined Monge-Ampère measure on $\tilde{X}$. Since $\tilde{\varphi}$ is bounded and $\tilde{\omega}$-plurisubharmonic, by the work of Bedford and Taylor [BT76], it puts no mass on the exceptional locus $\pi^{-1}\left(X_{\text {sing }}\right)$. Furthermore,

$$
\int_{X \backslash X_{\text {sing }}}\left(\omega+\frac{i}{2} \partial \bar{\partial} \varphi\right)^{n}=\int_{\tilde{X}}\left(\tilde{\omega}+\frac{i}{2} \partial \bar{\partial} \tilde{\varphi}\right)^{n}=\int_{\tilde{X}} \tilde{\omega}^{n}<\infty .
$$

Therefore, $\left(\tilde{\omega}+\frac{i}{2} \partial \bar{\partial} \tilde{\varphi}\right)^{n}$ can be pushed forth to a measure on $X$ and it coincides with the trivial extension of $\left(\omega+\frac{i}{2} \partial \bar{\partial} \varphi\right)^{n}$ from $X \backslash X_{\text {sing }}$ to $X$. In particular, the Monge-Ampère measure $\left(\omega+\frac{i}{2} \partial \bar{\partial} \varphi\right)^{n}$ on $X$ does not depend on the resolution of singularities.

Let $\Omega$ be a smooth real valued semi-positive $(n, n)$-form on $X \backslash X_{\text {sing }}$ and let $\tilde{\Omega}=\pi^{*} \Omega$. We consider the following Monge-Ampère equation on $X$

$$
\left(\omega+\frac{i}{2} \partial \bar{\partial} \varphi\right)^{n}=e^{\alpha \varphi+F} \Omega
$$

where $\alpha=-1,0,1$ and $F$ is a real valued function on $X$. This Monge-Ampère equation can be lifted to a Monge-Ampère equation on $\tilde{X}$ if the solution $\varphi$ is bounded and $\omega$ plurisubharmonic, i.e., $\omega+\frac{i}{2} \partial \bar{\partial} \varphi \geq 0$. More precisely, let $\tilde{\varphi}=\pi^{*} \varphi$, and consider the Monge-Ampère equation on $\tilde{X}$

$$
\left(\tilde{\omega}+\frac{i}{2} \partial \bar{\partial} \tilde{\varphi}\right)^{n}=e^{\alpha \tilde{\varphi}+\tilde{F}} \tilde{\Omega}
$$

where $\tilde{F}=\pi^{*} F$. If $\varphi$ is a bounded $\omega$-plurisubharmonic solution of equation (10.4), then $\tilde{\varphi}=\pi^{*} \varphi$ is bounded and $\tilde{\omega}$-plurisubharmonic, and it solves equation (10.5). Now let us assume that $\tilde{\varphi}$ is a bounded $\tilde{\omega}$-plurisubharmonic solution of the equation (10.5). Any fibre of $\pi$ over a singular point of $X$ is connected by Zariski's connectedness theorem, and $\tilde{\omega}=0$ when restricted to the fibre. Therefore $\tilde{\varphi}$ is constant along the fibre because it is plurisubharmonic and bounded. Hence $\tilde{\varphi}$ descends to a function $\varphi$ on $X$. The function $\varphi$ is bounded and $\omega$-plurisubharmonic on $X \backslash X_{\text {sing }}$, and so it is $\omega$-plurisubharmonic function on $X$ because $X$ is normal. We have thus shown

Lemma 14 The equation (10.4) admits a bounded $\omega$-plurisubharmonic solution $\varphi$ if and only if the equaiton (10.5) admits a bounded $\tilde{\omega}$-plurisubharmonic solution $\tilde{\varphi}$. 
Therefore, we can solve equation (10.5) on a smooth manifold $\tilde{X}$ instead of solving equation (10.4) on a singular variety $X$. Furthermore the construction is resolution independent because given any two resolutions, we can move the measures to the same resolution and apply the uniqueness property of Monge-Ampère equations there. So we obtain the following lemma, which follows immediately from Theorem 5:

Lemma 15 Let $\Theta$ be a smooth volume form on $\tilde{X}$. Then the equation (10.4) admits a bounded and $\omega$-plurisubharmonic solution for $\alpha=0$, if $\frac{e^{\tilde{F}} \tilde{\Omega}}{\Theta} \in L^{p}(\tilde{X})$ for some $p>1$.

In fact, Lemma 15 also holds for $\alpha=1$ by [EGZ]. We can apply it now to solving Kähler-Einstein equations on singular varieties. Recall some basic definitions for canonical models of general type:

Definition 1 (a) A projective variety $X$ is said to be a canonical model of general type if $X$ is a normal and the canonical divisor $K_{X}$ is an ample $\mathbf{Q}$-line bundle.

(b) Let $X$ be a canonical model of general type. A form $\Omega$ is said to be a smooth volume form on $X$ if for any point $z \in X$, there exists an open neighborhood $U$ of $z$ such that

$$
\Omega=f_{U}(\eta \wedge \bar{\eta})^{\frac{1}{m}}
$$

where $f_{U}$ is a smooth positive function on $U$ and $\eta$ is a local generator of $m K_{X}$ on $U$. In particular, any smooth volume $\Omega$ induces a smooth hermitian metric $h=\Omega^{-1}$ on $K_{X}$.

(c) $X$ is said to be a canonical model of general type with canonical singularities if for any resolution of singularities $\pi: \tilde{X} \rightarrow X$ and any smooth volume form $\Omega$ on $X$,

$$
\tilde{\Omega}=\pi^{*} \Omega
$$

is a smooth real valued $(n, n)$-form on $\tilde{X}$.

We can now describe some recent results of Eyssidieux, Guedj, and Zeriahi [EGZ] on the existence of Kähler-Einstein metrics of zero or negative curvature on manifolds with canonical singularities. As above, let $\pi: \tilde{X} \rightarrow X$ be a resolution of singularities, let $m \omega$ be the restriction of the Fubini-Study metric of $\mathbf{C P}^{M}$ on $X$, and let $\Omega$ be a smooth volume form $\Omega$ on $X$ such that

$$
\frac{i}{2} \partial \bar{\partial} \log \Omega=\omega
$$

The following theorem on Kähler-Einstein metrics with negative curvature was proved in [EGZ], using the $C^{0}$ estimates of Theorem 5:

Theorem 16 Let $X$ be a canonical model of general type with canonical singularities. Then there exists a unique bounded and $\omega$-plurisubharmonic function $\varphi$ solving the following Monge-Ampère equation on $X$ 


$$
\left(\omega+\frac{i}{2} \partial \bar{\partial} \varphi\right)^{n}=e^{\varphi} \Omega
$$

In particular, on $X \backslash X_{\text {sing }}, \omega_{K E}=\omega+\frac{i}{2} \partial \bar{\partial} \varphi$ is smooth and

$$
\operatorname{Ricci}\left(\omega_{K E}\right)=-\omega_{K E} .
$$

Next we discuss the case of zero curvature. Recall that $X$ is said to be a Calabi-Yau variety if $X$ is a projective normal variety and $m K_{X}$ is a trivial line bundle on $X$ for some $m \in \mathbf{Z}^{+}$. Since $m K_{X}$ is a trivial line bundle on $X$, there exists a constant global section

$\eta$ of $m K_{X}$. Let $\Omega_{C Y}=(\eta \wedge \eta)^{\frac{1}{m}}$. Then $\Omega$ is a smooth volume form on $X$. Furthermore, $\frac{i}{2} \partial \bar{\partial} \log \Omega_{C Y}=0$.

Definition 2 A Calabi-Yau variety $X$ is said to be a Calabi-Yau variety with canonical singularities if for any resolution of singularities $\pi: \tilde{X} \rightarrow X$,

$$
\tilde{\Omega}_{C Y}=\pi^{*} \Omega_{C Y}
$$

is a smooth real valued $(n, n)$-form on $\tilde{X}$.

Let $X$ be a Calabi-Yau variety with canonical singularities. We choose the smooth Kähler form $\omega_{L} \in c_{1}(L)$ induced from the Fubini-Study metric on $\mathbf{C P}^{N}$ in the same way as in the earlier discussion. Then we have the following theorem due to [EGZ]

Theorem 17 Let $X$ be a Calabi-Yau variety with canonical singularities. Then for any ample Q-line bundle, there exists a unique bounded and $\omega_{L}$-plurisubharmonic function $\varphi$ solving the following Monge-Ampère equation on $X$

$$
\left(\omega_{L}+\frac{i}{2} \partial \bar{\partial} \varphi\right)^{n}=c_{L} \Omega_{C Y}
$$

where $c_{L} \int_{X} \Omega_{C Y}=\int_{X} \omega_{L}^{n}$. In particular, on $X \backslash X_{\text {sing }}, \omega_{C Y}=\omega_{L}+\frac{i}{2} \partial \bar{\partial} \varphi$ is smooth and

$$
\operatorname{Ricci}\left(\omega_{C Y}\right)=0
$$

\subsection{Positivity notions for cohomology classes}

Another extension of the theory is the existence of Kähler-Einstein metrics, which are then necessarily singular, on manifolds $X$ whose first Chern class $c_{1}\left(K_{X}\right)$ is neither zero nor positive definite.

To discuss the classes which are allowed, we recall briefly the definitions of some basic cones in the space of cohomology classes. They were introduced by Demailly [D1] and play an important role in his differential geometric approach to positivity problems in algebraic 
geometry. Let $X$ be a compact Kähler manifold and $\alpha \in H^{1,1}(X, \mathbf{R})$ be a cohomology class. Then

$$
\alpha \in \frac{\{\theta: \text { closed }(1,1) \text { forms }\}}{\{\theta: \text { exact }(1,1) \text { forms }\}}=\frac{\{T: \text { closed }(1,1) \text { currents }\}}{\{T: \text { exact }(1,1) \text { currents }\}}
$$

We say $\alpha$ is pseudo-effective (psef) if there is a closed $(1,1)$ current $T \in \alpha$ such that $T \geq 0$. We say $\alpha$ is big if there exists $T \in \alpha$ with $T \geq \varepsilon \omega$ for some $\varepsilon>0$ and some Kähler form $\omega$.

Let $\operatorname{PSEF}(X)$ be the set of psef classes and $\operatorname{BIG}(X)$ the set of big classes. Then we clearly have $\operatorname{BIG}(X) \subseteq \operatorname{PSEF}(X)$. Moreover, $\operatorname{PSEF}(X)$ is a closed convex cone in the vector space $H^{1,1}(X, \mathbf{R})$ and $\operatorname{BIG}(X)$ is an open convex cone. If $T$ is psef then $T+\varepsilon \omega$ is big for all $\varepsilon>0$. This shows that $\operatorname{BIG}(X)$ is precisely the interior of $\operatorname{PSEF}(X)$.

Let $\operatorname{KAH}(X)$ be the set of Kähler classes in $H^{1,1}(X, \mathbf{R})$. Thus $\alpha \in \operatorname{KAH}(X)$ if and only if there exists a Kähler form $\omega \in \alpha$. Thus $\operatorname{KAH}(X)$ is an open cone and we clearly have $\mathrm{KAH}(X) \subseteq \mathrm{BIG}(X)$. This inclusion may be proper. Let $\mathrm{NEF}(X)$ be the closure of $\operatorname{KAH}(X)$. An element of $\alpha \in \operatorname{NEF}(X)$ is called a nef class. In summary,

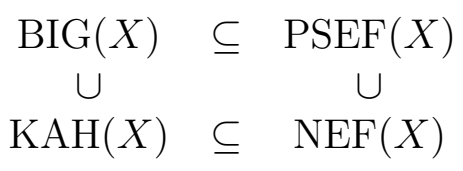

The cones on the left are open and those on the right are their closures.

Let

$$
\mathcal{T}(X, \alpha)=\{T \in \alpha: T \text { a closed }(1,1) \text { current, } T \geq 0\}
$$

Then $\alpha$ is pseudo-effective (psef) iff $\mathcal{T}(X, \alpha) \neq \emptyset$. We endow $\mathcal{T}(X, \alpha)$ with the weak topology, so that $T_{j} \rightarrow T$ iff $\int_{X} T_{j} \wedge \eta \rightarrow \int_{X} T \wedge \eta$ for all smooth $(n-1, n-1)$ forms $\eta$. The space $\mathcal{T}(X, \alpha)$ is compact in the weak topology.

Fix a smooth volume form $d V$ on $X$ and let $L^{1}(X)=L^{1}(X, d V)$. For $\theta \in \alpha$ smooth, let $P S H^{1}(X, \theta)=\left\{\varphi \in L^{1}(X): \theta+\frac{i}{2} \partial \bar{\partial} \varphi \geq 0\right\}$ endowed with the $L^{1}(X)$ topology. The map $\varphi \mapsto \theta+\frac{i}{2} \partial \bar{\partial} \varphi$ defines $P S H^{1}(X, \theta) / \mathbf{R} \rightarrow \mathcal{T}(X,[\theta])$ a homeomorphism of compact topological spaces. The map sup : $P S H^{1}(X, \theta) \rightarrow \mathbf{R}$ is continuous (this is Hartogs' lemma). Thus we have a homeomorphism

$$
\left\{\varphi \in P S H^{1}(X, \theta): \sup \varphi=0\right\} \rightarrow \mathcal{T}(X,[\theta])
$$

Let $X$ be a compact Kähler manifold and $\alpha \in H^{1,1}(X, \mathbf{R})$ a big class. Fix $\theta \in \alpha$, a closed smooth $(1,1)$ form. Then, by definition, there exists $\varphi \in P S H^{1}(X, \theta)$ such that $\theta+\frac{i}{2} \partial \bar{\partial} \varphi \geq \varepsilon \omega$ for some Kähler metric $\omega$ and some $\varepsilon>0$. Demailly's theorem says that we may choose $\varphi$ such that $\varphi$ has analytic singularities. This means that locally on $X$,

$$
\varphi=c \log \left(\sum_{j=1}^{N}\left|f_{j}\right|^{2}\right)+\psi
$$


where $c>0, f_{j}$ are holomorphic and $\psi$ is smooth. In particular, the set where $T$ is smooth is a Zariski open subset of $X$.

Let $X$ and $\theta$ be as above. Thus $\theta$ is big, but it general it will not be positive. We define $V_{\theta} \in P S H(X, \theta)$, the extremal function of $\theta$ (the analogue of the "convex hull") by

$$
V_{\theta}(x)=\sup \left\{\varphi(x): \varphi \in P S H^{1}(X, \theta), \sup _{X} \varphi \leq 0\right\}
$$

Thus $V_{\theta}=0$ if $\theta$ is a Kähler metric.

The extremal function $V_{\theta}$ has a number of nice properties. To describe them, and because $V_{\theta}$ is not bounded in general, we need to extend the definition of Monge-Ampère measures. We shall use the definition that does not charge pluripolar sets, and which can be described as follows.

Let $T_{1}, \ldots, T_{p}$ be closed positive $(1,1)$ currents. For any $z \in M$ there there exists an open set $U$ containing $z$, and pluri-subharmonic functions $u_{1}, \ldots, u_{j}$, for which $T_{j}=\frac{i}{2} \partial \bar{\partial} u_{j}$. Let $U_{k}=\cap_{j}\left\{u_{j}>-k\right\} \subseteq U$. Then the non-pluripolar product $\left\langle T_{1} \wedge \cdots \wedge T_{p}\right\rangle$ is the closed $(p, p)$-current defined by [BEGZ]

$$
\left.T_{1} \wedge \cdots \wedge T_{p}\right|_{U}=\lim _{k \rightarrow \infty} \mathbf{1}_{U_{k}} \bigwedge_{j=1}^{p} \frac{i}{2} \partial \bar{\partial} \max \left(u_{j},-k\right)
$$

where $\mathbf{1}_{U_{k}}$ denotes the characteristic function of $U_{k}$. The non-pluripolar product coincides with the Bedford-Taylor definition of $T_{1} \wedge \cdots \wedge T_{p}$ if the potentials are all bounded. We still denote $T_{1} \wedge \cdots \wedge T$ by $T^{n}$. We can now describe the properties of extremal functions:

Theorem 18 Let $X$ be a compact Kähler manifold and $\theta$ a big $(1,1)$ form. Let $V_{\theta}$ be the extremal function of $\theta$. Then

(1) $V_{\theta} \in P S H^{1}(X, \theta)$

(2) $V_{\theta}$ has minimal singularities: if $\varphi \in P S H^{1}(X, \theta)$ then $\varphi \leq V_{\theta}+C$ for some $C \geq 0$.

(3) On the set where $\theta$ is smooth, $V_{\theta}$ is continuous, and $\frac{i}{2} \partial \bar{\partial} V_{\theta}$ is locally bounded.

(4) $\left(\frac{i}{2} \partial \bar{\partial} V_{\theta}\right)^{n}$ has $L^{\infty}$ density with respect to $d V$. In particular, if $\varphi \in P S H^{1}(X, \theta)$ then $\int_{X}|\varphi|\left(\frac{i}{2} \partial \bar{\partial} V_{\theta}\right)^{n}<\infty$.

(5) $V_{\theta}$ is maximal with respect to $\theta$, that is

$$
\left(\frac{i}{2} \partial \bar{\partial} V_{\theta}\right)^{n}=\mathbf{1}_{\left\{V_{t}=0\right\}} \theta^{n}
$$

\subsection{Prescribing the Monge-Ampère measure}

If $[\theta]$ is a big class and $T_{1}, \ldots, T_{n} \in \mathcal{T}(X,[\theta])$ then $\int_{X}\left\langle T_{1} \wedge \cdots \wedge T_{n}\right\rangle \leq \int_{X}[\theta]^{n}$. If we have $T_{1}=\cdots T_{n}=T=\theta+\frac{i}{2} \partial \bar{\partial} \varphi$ and if equality holds, we say $T$ has full mass. Define

$$
\begin{aligned}
& \mathcal{T}^{0}(X, \theta)=\{T \in T(X, \theta): T \text { has full mass }\} \\
& \mathcal{T}^{1}(X, \theta)=\left\{T=\theta+\frac{i}{2} \partial \bar{\partial} \varphi \in T^{1}(X, \omega): \int_{X}|\varphi|\left(\theta+\frac{i}{2} \partial \bar{\partial} \varphi\right)^{n}<\infty\right\}
\end{aligned}
$$


Let $\mathcal{M}_{X}$ be the space of probability measures on $X$, let $\mathcal{M}_{X}^{0}$ consist of those measures which take no mass on pluri-polar sets, and let $\mathcal{M}_{X}^{1}$ consist of those measures of "finite energy" (to be defined below). Then it has been shown by Guedj and Zeriahi [GZ] and by Berman, Boucksom, Guedj, and Zeriahi [BBGZ] respectively that the map $T \mapsto T^{n}$ defines bijections:

$$
\mathcal{T}^{0}(X, \omega) \rightarrow \mathcal{M}_{X}^{0}, \quad \mathcal{T}^{1}(X, \omega) \rightarrow \mathcal{M}_{X}^{1} .
$$

\subsection{Singular KE metrics on manifolds of general type}

Before stating the results, it is convenient to recast the Kähler-Einstein equation for negative curvature in a slightly different form from usual. Let $X$ be a compact Kähler manifold, and let $K_{X}$ be its canonical bundle. If $c_{1}\left(K_{X}\right)$ is a Kähler class, then the Aubin-Yau theorem says that for any Kähler metric $\omega$ there is a unique smooth $\psi \in P S H(X,-\operatorname{Ricci}(\omega))$ such that

$$
\left(-\operatorname{Ricci}(\omega)+\frac{i}{2} \partial \bar{\partial} \psi\right)^{n}=e^{\psi} \omega^{n}
$$

To put this in the usual form, let $\eta=-\operatorname{Ricci}(\omega)+\frac{i}{2} \partial \bar{\partial} \psi$. Then $\eta>0$ and

$$
\operatorname{Ricci}(\omega)-\operatorname{Ricci}(\eta)=\frac{i}{2} \partial \bar{\partial} \psi=\operatorname{Ricci}(\omega)+\eta
$$

which implies $\operatorname{Ricci}(\eta)=-\eta$.

Now assume that $c_{1}(K)$ is big and nef. Then Tsuji [Ts] proved that there is a subvariety $Z \subseteq X$ and a smooth function $\psi \in C^{\infty}(X \backslash Z)$ such that $\eta=-\operatorname{Ricci}(\omega)+\frac{i}{2} \partial \bar{\partial} \psi>0$ and such that (10.21) holds on $X \backslash Z$. Thus Ricci $(\eta)=-\eta$ on $X \backslash Z$.

Tian-Zhang [TZ] proved that $\psi$ extends to a locally bounded $\psi \in P S H(X,-\operatorname{Ricci}(\omega))$ satisfying $\int\left(-\operatorname{Ricci}(\omega)+\frac{i}{2} \partial \bar{\partial} \psi\right)^{n}=\int(-\operatorname{Ricci}(\omega))^{n}$ (i.e., $\psi$ has full MA measure) and that (10.21) holds on all of $X$.

Now assume that $c_{1}(X)$ is big. Then [EGZ] showed that there is a unique $\psi \in$ $P S H^{1}(-\operatorname{Ricci}(\omega))$ of full Monge-Ampère measure such that (10.21) holds. The [EGZ] proof uses the existence of canonical models. Tsuji described an interesting approach to proving the existence of a singular Kähler-Einstein metric without resorting to the existence of a canonical model in [Ts]. Then Song-Tian [ST09] gave an independent proof, via the Kähler-Ricci flow. A new proof was also given by [BEGZ] which used a generalized comparison principle. More recently, a proof using variational methods was given in [BBGZ].

\section{Variational Methods for Big Cohomology Classes}

A basic property of the Monge-Ampère determinant is that it can be interpreted as the variational derivative of a concave energy functional. In fact, if $\omega$ is a smooth Kähler form 
on a compact complex manifold $X$, and we set

$$
E_{\omega}(\varphi)=\frac{1}{n+1} \sum_{j=0}^{n} \int_{X} \varphi\left(\omega+\frac{i}{2} \partial \bar{\partial} \varphi\right)^{j} \wedge \omega^{n-j}
$$

for $\omega_{\varphi} \equiv \omega+\frac{i}{2} \partial \bar{\partial} \varphi>0$. Then for smooth and small variations $\delta \varphi$, we have

$$
\delta E=\int_{X} \delta \varphi \omega_{\varphi}^{n} \text { and } \delta^{2} E=-n \int_{X} d \delta(\varphi) \wedge d^{c} \delta \varphi \wedge \omega_{\varphi}^{n-1}
$$

This shows that $\frac{\delta E}{\delta \varphi}=\omega_{\varphi}^{n}$ and that $E$ is concave.

The functional $E(\varphi)$ is actually equal to $E(\varphi)=-\left(J(\varphi)-\int_{X} \varphi \omega^{n}\right)$, where

$$
J(\varphi)=\sum_{j=0}^{n-1} \frac{n-j}{n+1} \int_{X} i \partial \varphi \wedge \bar{\partial} \varphi \wedge \omega_{\varphi}^{n-1-j} \wedge \omega^{j} .
$$

This relation will play an important role below The functional $E$ is sometimes denoted by $-(n+1) F^{0}$ in the literature, where $F^{0}$ is the Aubin-Yau energy functional.

The goal of this section is to describe some recent work of Berman, Boucksom, Guedj, and Zeriahi [BBGZ] taking this variational viewpoint further. Their approach, which works in the generality of big cohomology classes, allows one to use direct methods of the calculus of variations to obtain solutions to a variety of Monge-Ampère equations.

\subsection{Finite dimensional motivation}

We start with a finite dimensional model for the method introduced in [BBGZ]. Let $P \subseteq \mathbf{R}^{n}$ be a convex domain and $E: P \rightarrow \mathbf{R}$ be a strictly concave smooth function. Then $\nabla E: T P \rightarrow \mathbf{R}$ where $T P=P \times \mathbf{R}^{n}$ is the tangent bundle of $P$. In other words, $\nabla E: P \rightarrow\left(\mathbf{R}^{n}\right)^{*}$ where $\left(\mathbf{R}^{n}\right)^{*}$ is the dual of $\mathbf{R}^{n}$. Since $E$ is strictly concave, $\nabla^{2} E(\varphi)$ : $\mathbf{R}^{n} \rightarrow\left(\mathbf{R}^{n}\right)^{*}$ is strictly negative definite for all $\varphi \in P$ and $\nabla E$ is a diffeomorphism of $P$ onto a convex domain $\mathcal{M} \subseteq\left(\mathbf{R}^{n}\right)^{*}$.

More generally, suppose $E$ is concave (but not strictly concave) and that there is a flat direction, that is, an element $\varphi_{0} \in P$ such that $E\left(\varphi+t \varphi_{0}\right)=E(\varphi)+t$ for all $t \in \mathbf{R}$ and let $\mathcal{T}^{1}=P /\left(\mathbf{R} \cdot \varphi_{0}\right)$ which we view as a domain in the vector space $W=\mathbf{R}^{n} /\left(\mathbf{R} \cdot \varphi_{0}\right)$. Then $\nabla E: P \rightarrow\left(\mathbf{R}^{n}\right)^{*}$ is invariant under $\mathbf{R} \cdot \varphi_{0}$ so

$$
\nabla E: \mathcal{T}^{1} \rightarrow \mathcal{M}^{0}
$$

where $\mathcal{M}^{0} \subseteq\left(\mathbf{R}^{n}\right)^{*}$ is the $n-1$ dimensional affine space

$$
\mathcal{M}^{0}=\left\{\mu \in \mathbf{R}^{n}:\left\langle\varphi_{0}, \mu\right\rangle=1\right\}=\text { the elements of }\left(\mathbf{R}^{n}\right)^{*} \text { of full mass }
$$

Now we want to impose the following condition: $E$ is strictly concave in every direction other than $\mathbf{R} \cdot \varphi_{0}$. There are several equivalent ways of making this condition precise: 
Lemma 16 Let $E: P \rightarrow \mathbf{R}$ be a concave function and assume that $E\left(\varphi+t \varphi_{0}\right)=E(\varphi)+t$ for all $t \in \mathbf{R}$. Then the following conditions are equivalent

(1) The map $t \mapsto E\left((1-t) \psi_{0}+t \psi_{1}\right)$ is strictly concave whenever $\psi_{1}-\psi_{0} \notin \mathbf{R} \cdot \varphi_{0}$.

(2) The negative definite map $\nabla^{2} E(\varphi): W \rightarrow W^{*}$ is strictly negative definite if $\varphi \in \mathcal{T}$.

(3) The map $\nabla E: \mathcal{T}^{1} \rightarrow \mathcal{M}$ is a diffeomorphism where $\mathcal{M} \subseteq \mathcal{M}^{0}$ is the image of $\nabla E$.

(4) Fix $\psi_{0} \in \mathcal{T}^{1}$, let $\mu_{0}=\nabla E\left(\psi_{0}\right), L_{0}(\varphi)=\varphi \cdot \mu_{0}$ and define $J_{0}: \mathcal{T}^{1} \rightarrow[0, \infty)$ by

$$
J_{\psi_{0}}(\varphi)=E\left(\psi_{0}\right)+\nabla E\left(\varphi_{0}\right) \cdot\left(\varphi-\psi_{0}\right)-E(\varphi)=L_{0}(\varphi)-E(\varphi)+C
$$

Then $J_{0}=J_{\psi_{0}}: \mathcal{T}^{1} \rightarrow[0, \infty)$ is strictly convex

The proof of the lemma is easy and will be omitted. Henceforth, we shall assume that $E$ satisfies any one of the equivalent conditions enumerated in Lemma 1.

The function $J_{0}$ is proper so the sets $\left\{J_{0} \leq C\right\} \subseteq \mathcal{T}^{1}$ are compact and exhaust $\mathcal{T}^{1}$. In the case $\mathcal{T}^{1}$ is bounded, we can define $\mathcal{T}$ to be the closure of $\mathcal{T}^{1}$ and extend $J_{0}: \mathcal{T} \rightarrow[0, \infty]$ as a continuous map between compact spaces.

For $\mu \in \mathcal{M}^{0}$ we let

$$
E^{*}(\mu)=\sup _{\varphi \in \mathcal{T}}(E(\varphi)-\varphi \cdot \mu)=\sup _{\varphi \in \mathcal{T}} F_{\mu}(\varphi) \in(-\infty, \infty]
$$

We let $\mathcal{M}^{1} \subseteq \mathcal{M}^{0}$ be the elements with finite energy:

$$
\mathcal{M}^{1}=\left\{\mu \in \mathcal{M}^{0}: E^{*}(\mu)<\infty\right\}
$$

If $\mu \in \mathcal{M}$ then, by definition, there exists $\varphi \in \mathcal{T}^{1}$ such that $\nabla E(\varphi)=\mu$ and hence $\varphi$ is a critical point of $F_{\mu}$. Since $F_{\mu}$ is strictly concave we conclude that $F_{\mu}$ achieves its maximum at $\varphi$ and thus

$$
\mathcal{M} \subseteq \mathcal{M}^{1} \subseteq \mathcal{M}^{0}
$$

Conversely, if $\mu \in \mathcal{M}^{1}$ then $\sup F_{\mu}<\infty$ and $\mu \in \mathcal{M}$ if and only if $F_{\mu}$ achieves its sup (i.e. there exists $\varphi \in \mathcal{T}^{1}$ such that $\left.F_{\mu}(\varphi)=E^{*}(\mu)\right)$.

The inclusion $\mathcal{M} \subseteq \mathcal{M}^{1}$ may be strict. We give two simple examples:

Example A. Suppose $\mathcal{T}=\mathbf{R}$ and $E: \mathbf{R} \rightarrow \mathbf{R}$ is smooth and concave, and $E(x)=$ $\log x-x$ for $x$ large. Then $\mathcal{M}=\mathcal{M}^{1}=(-1,1)$. So for example A,

$$
\mathcal{M}=\mathcal{M}^{1}
$$

Example B. Suppose $\mathcal{T}=\mathbf{R}$ and $E: \mathbf{R} \rightarrow \mathbf{R}$ is smooth and convex, and $E(x)=-x-\frac{1}{x}$ for $x$ large. Then $\mathcal{M}=(-1,1)$ but $\mathcal{M}^{1}=[-1,1]$. Thus in example B property (11.9) fails. 
There is a simple criterion for guaranteeing that (11.9) holds. Suppose $J: \mathcal{T} \rightarrow[0, \infty)$ is a proper function and let $\mu \in \mathcal{M}^{1}$. We say that $F_{\mu}$ is coercive with respect to $J$ if there exists $\varepsilon, C>0$, depending on $\mu$, such that

$$
L_{\mu}-E=-F_{\mu} \geq \varepsilon J-C=\varepsilon\left(L_{\mu_{0}}-E\right)-C^{\prime}
$$

We say $F$ is $J$-proper if $-F_{\mu} \rightarrow \infty$ as $J \rightarrow \infty$. Clearly $J$-coercive implies $J$-proper. Note that (11.10) holds trivially if $\varepsilon=0$ : If $\mu_{0}=\nabla E\left(\psi_{0}\right)$ then $J_{0}(\varphi)=F_{\mu_{0}}\left(\psi_{0}\right)-F_{\mu_{0}}(\varphi) \geq 0$.

Lemma 17 Let $\mu \in \mathcal{M}^{1}$ and assume that $F_{\mu}$ is $J$-proper for some $J$. Then $\mu \in \mathcal{M}$.

To see this, we fix $\varphi_{0} \in \mathcal{T}$ and let $A=J\left(\varphi_{0}\right)+1$. Then the set $\mathcal{T}_{A}=\{J \leq A\}$ is compact so there exists $\hat{\varphi}$ such that $F_{\mu}(\hat{\varphi}) \geq F_{\mu}(\varphi)$ for all $\varphi \in \mathcal{T}_{A}$ and hence for all $\varphi \in \mathcal{T}$. Thus $F_{\mu}$ achieves its sup at $\hat{\varphi}$ which implies $\nabla F_{\mu}(\hat{\varphi})=0$, that is, $\nabla E(\hat{\varphi})=\mu$. This shows $\mu \in \mathcal{M}$.

In order to apply Lemma 17 we need to find an appropriate $J$. It turns out that if $F_{\mu}$ is $J$-coercive for some $J$, then it is coercive for the function $J_{0}$ constructed in (11.6):

Lemma 18 Let $\mu \in \mathcal{M}^{1}$. Then the following are equivalent

(1) $\mu \in \mathcal{M}$

(2) $F_{\mu}$ is $J_{0}$-coercive.

(3) $F_{\mu}$ is $J$-coercive for some exhaustion function $J$.

(4) $F_{\mu}$ is $J$-proper for some exhaustion function $J$.

Proof. We need only show that 1 ) implies 2). Let $\mu \in \mathcal{M}$. We must show that for some $\varepsilon, C>0$ the following holds.

$$
-F_{\mu}(\varphi) \geq \varepsilon\left(F_{\mu_{0}}\left(\psi_{0}\right)-F_{\mu_{0}}(\varphi)\right)-C
$$

which we rewrite as

$$
\varphi \cdot \mu_{\varepsilon}-E(\varphi)=\varphi \cdot \frac{\left(\mu-\varepsilon \mu_{0}\right)}{1-\varepsilon}-E(\varphi) \geq \frac{\varepsilon F_{\mu_{0}}\left(\psi_{0}\right)-C}{1-\varepsilon}
$$

But $\mathcal{M}$ is an open set so for $\varepsilon$ sufficiently small, $\mu_{\varepsilon} \in \mathcal{M}$ which implies $\varphi \cdot \mu_{\varepsilon}-E(\varphi)$ is bounded below.

Now fix $\psi_{0} \in \mathcal{T}$ and define $J_{0}$ as in (11.6).

Lemma $19 \mathcal{M}=\mathcal{M}^{1}$ if and only if $F_{\mu}$ is $J_{0}$-coercive for all $\mu \in \mathcal{M}^{1}$.

Now we record a condition on $J_{0}$ that guarantees $F_{\mu}$ is coercive for all $\mu$. This key condition will hold in the infinite dimensional setting and is used in the proof of coerciveness.

Lemma 20 Suppose that $J_{0}$ grows quadratically or, more generally, suppose that

$$
\left|J_{0}(t \varphi)\right| \leq C\left(t^{p}|J(\varphi)|+1\right) \text { if } t \leq 1
$$

for some $p>1$. Then $F_{\mu}$ is $J_{0}$-coercive for all $\mu \in \mathcal{M}^{1}$. 
Observe that we can rewrite the coercive condition (11.10) as follows:

$$
L_{\mu_{0}}-L_{\mu} \leq(1-\varepsilon) J_{0}+C_{1}
$$

Now (11.13) implies that for arbitrary $\varphi \in \mathcal{T}$, we have $\varphi_{p}=\frac{\varphi}{|J(\varphi)|^{1 / p}} \in\left\{J_{0} \leq C_{2}\right\}$ which is a compact set. This implies

$$
\frac{\left|L_{\mu_{0}}-L_{\mu}\right|(\varphi)}{\left|J_{0}(\varphi)\right|^{1 / p}}=\left|L_{\mu_{0}}-L_{\mu}\right|\left(\varphi_{p}\right) \leq C_{3}
$$

which clearly implies (11.14).

\subsection{The infinite dimensional setting}

We describe the work of [BBGZ]. Let $\theta$ be a big $(1,1)$ form on a compact Kähler manifold $X$. Let $P=P S H^{1}(X, \theta)$ and $\mathcal{T}=\mathcal{T}(X, \theta)$ and $\mathcal{M}^{\prime}$ the space of positive metrics on $X$. Our goal is to define a concave function $E: P S H^{1}(X, \theta) \rightarrow \mathbf{R}$ with the property: $\nabla E: P \rightarrow \mathcal{M}^{\prime}$ is the map $\varphi \mapsto M A(\varphi)$, where $M A(\varphi)$ is the Monge-Ampère measure, defined by the non-pluripolar product. Moreover, we will show that if $\varphi_{0}=1$, the constant function, then $\varphi_{0}$ is a flat direction for $E$ and $\mathcal{T}$ is a complement to $\mathbf{R} \varphi_{0}$ in $P$. Then, as in the finite dimensional case, we obtain a map $\nabla E: \mathcal{T} \rightarrow \mathcal{M}^{\prime}$. Also, in analogy with the finite dimensional case, we define the function $F_{\mu}(\varphi)=E(\varphi)-\int_{X} \varphi d \mu$ for $\mu \in \mathcal{M}^{\prime}$. Our goal is to prove (11.9) and this will be done by establishing (11.10) for a suitably chosen $J$.

The functional $E(\varphi)$ has been defined in (11.1) for $\theta=\omega$ a Kähler form, and $\varphi$ a smooth form in $P S H^{1}(X, \theta)$. To extend it to $\theta$ a big form and $\varphi \in P S H^{1}(X, \theta)$, we proceed as follows: Define $E(\varphi)=\inf \left\{E(\psi): \psi \in P S H^{1}(X, \omega) \cap C^{\infty}(X)\right.$ and $\left.\psi \geq \varphi\right\}$.

Now let $\theta$ be a big $(1,1)$ form (not necessarily Kähler). Let $\varphi \in P S H^{1}(X, \theta)$ and assume $\varphi$ has minimal singularities (i.e. that $\varphi-V_{\theta}$ is bounded). Define

$$
E(\varphi)=\frac{1}{n+1} \sum_{j=0}^{n} \int_{X}\left(\varphi-V_{\theta}\right)\left(\theta+\frac{i}{2} \partial \bar{\partial} \varphi\right)^{j}\left(\theta+\frac{i}{2} \partial \bar{\partial} V_{\theta}\right)^{n-j}
$$

Of course this coincides with (11.1) in the case where $\theta=\omega$ is Kähler (since in that case, $V_{\theta}=0$ ). If $\varphi$ is arbitrary, then again extend using the monotonicity property of $E$ as before: $E(\varphi)=\inf \{E(\psi): \psi \geq \varphi$ and $\psi$ has minimal singularities $\}$.

We wish to implement the finite dimensional program in the infinite dimensional setting. Since $E$ may assume the value $-\infty$, we must, at the outset, restrict $E$ to the set $\mathcal{E}^{1}=\left\{\varphi \in P S H^{1}(X, \theta):|E(\varphi)|<\infty\right\}$ so that $E: \mathcal{E}^{1} \rightarrow \mathbf{R}$ is concave. If $\varphi_{0}=1$, then $\varphi_{0}$ is a flat direction so, as in the finite dimensional program, we let $\mathcal{T}^{1}=\mathcal{E}^{1} / \mathbf{R}$ Again, we let $F_{\mu}(\varphi)=E(\varphi)-\int_{X}\left(\varphi-V_{\theta}\right) d \mu=E(\varphi)-L_{\mu}(\varphi)$ and let $\mathcal{M}^{1} \subseteq \mathcal{M}^{0}$ be those measures with finite energy. 


\subsection{Statement of theorems and sketch of proofs}

Theorem 19 ([BBGZ]) Let $\theta$ be a big $(1,1)$ form on a compact complex manifold $X$. If $\varphi \in \mathcal{T}^{1}(X, \theta)$ then $\nabla E(\varphi)=M A(\varphi)$ has full mass and finite energy, that is, $M A(\varphi) \in$ $\mathcal{M}^{1}$. Conversely, if $\mu \in \mathcal{M}^{1}$ then there exists a unique $\varphi \in \mathcal{T}^{1}$ such that $M A(\varphi)=\mu$. Moreover, the solution $\varphi$ satisfies the following bound:

$$
\frac{1}{n} E^{*}(\mu) \leq J_{0}(\varphi) \leq n E^{*}(\mu)
$$

where $J_{0}=J_{\psi_{0}}$ is defined in (11.6).

We sketch the proof of the converse, using the finite dimensional program as our guide. Recall that

$$
\begin{aligned}
J_{\psi}(\varphi) & =E(\psi)-E(\varphi)+\int_{X}(\varphi-\psi) M A(\psi)= \\
& =\sum_{j=0}^{n-1} \frac{j+1}{n+1} \int_{X} \partial(\varphi-\psi) \wedge \bar{\partial}(\varphi-\psi) \wedge \theta_{\psi}^{j} \wedge \theta_{\varphi}^{n-1-j}
\end{aligned}
$$

The first equality is a definition and the second follows via integration by parts. In particular, we see

$$
n^{-1} J_{\psi}(\varphi) \leq J_{\varphi}(\psi) \leq n J_{\psi}(\varphi)
$$

Now we can establish the bound (11.17): Assume $\varphi \in \mathcal{E}^{1}$ and that $M A(\varphi)=T^{n}=\mu$, where $T=\theta+\frac{i}{2} \partial \bar{\partial} \varphi$. Then (11.18) and (11.19) imply

$$
\frac{1}{n} J_{0}(\varphi) \leq J_{\varphi}\left(V_{0}\right)=E(\varphi)+\int_{X}\left(V_{0}-\varphi\right) M A(\varphi)=E(\varphi)-\int_{X}\left(\varphi-V_{0}\right) d \mu=F_{\mu}(\varphi)
$$

Since $M A(\varphi)=\mu$ one shows, in analogy with the finite dimensional picture, that $E^{*}(\mu)=$ $\sup _{\psi \in \mathcal{E}^{1}} F_{\mu}(\psi)=F_{\mu}(\varphi)$. This proves (11.17).

Now let us fix $\mu \in \mathcal{M}^{1}$. We wish to prove the existence of $T \in \mathcal{T}^{1}$ such that $T^{n}=\mu$. The first step is to prove that $F_{\mu}$ is $J_{0}$ coercive, where $J_{0}$ is defined with respect to the potential $\psi_{0}=V_{0}$, as in (11.6). To do this, we wish to use Lemma 20, which means that we must prove that $E$ grows quadratically. But this follows easily from the definitions (here we assume $V_{\theta}=0$ for simplicity): If $\sup _{X} \varphi=0$ then

$$
|E(t \varphi)|=\frac{1}{n+1}\left|\sum_{j=0}^{n} \int(t \varphi)\left(t\left(\omega+\frac{i}{2} \partial \bar{\partial} \varphi\right)+(1-t) \omega\right)^{j} \omega^{n-j}\right| \leq c(n)\left[t^{2} E(\varphi)+1\right]
$$

For $C>0$ let $\mathcal{E}_{C}$ be the compact set $\mathcal{E}_{C}=\left\{\varphi \in \mathcal{E}^{1}: E(\varphi) \geq C\right\}$. Since $F_{\mu}$ is coercive, there exists $C>0$ such that $\sup _{\varphi \in \mathcal{E}^{1}} F_{\mu}=\sup _{\varphi \in \mathcal{E}_{\mathcal{C}}} F_{\mu}$. 
The first main difficulty in implementing the finite dimensional program is proving that $F_{\mu}$ is upper semi continuous on $\mathcal{E}_{C}$. To do this, [BBGZ] restrict first to the case where $\mu \in \mathcal{C}=\left\{\mu \in \mathcal{M}^{1}: \mu \leq A \cdot\right.$ Cap for some $\left.A>0\right\}$ (here Cap is the pluri-subharmonic capacity).

Fix $\mu \in C$. Since $E$ is easily seen to be usc, it suffices to show that $L_{\mu}$ is continuous. Let $T: K \rightarrow L^{1}(X, d \mu)$ be the map $T(\varphi)=\varphi-V_{\theta}$ (here $K \subseteq \mathcal{E}^{1}$ is any compact convex subset). It's not hard to show that $T(K)$ is closed and that it has a closed graph. If we could prove $T(K)$ is compact, then we would be done. To see this, let $\varphi_{j} \rightarrow \varphi$ in $K$. If $T(K)$ is compact then $T\left(\varphi_{j}\right) \rightarrow f$ (after passing to a subsequence). But the closed graph property implies $f=\varphi$. Thus $T\left(\varphi_{j}\right) \rightarrow T(\varphi)$ so $T$ is continuous which implies $L_{\mu}$ is continuous.

Instead of proving that $T(K)$ is compact, we prove something weaker, namely that $T(K) \subseteq L^{1}(X, d \mu)$ is "convex combination compact". Recall that if $B$ is a Banach space and if $T \subseteq B$ is closed and convex, then $T$ is convex combination compact if for every sequence $\tau_{1}, \tau_{2}, \ldots \in T$ there exists $\tau_{1}^{\prime}, \tau_{2}^{\prime}, \ldots \in T$ such that $\tau_{j}^{\prime}$ is a finite convex combination of $\tau_{j}, \tau_{j+1}, \ldots$ and such that $\tau_{j}^{\prime}$ converges. Observe that showing $T(K)$ is convex combination compact suffices for our purposes: Let $\varphi_{j} \rightarrow \varphi \in K$. Then one shows $L_{\mu}\left(\varphi_{j}\right)$ is bounded and hence $L_{\mu}\left(\varphi_{j}\right)=\int T\left(\varphi_{j}\right) \rightarrow \ell \in \mathbf{R}$ (after passing to a subsequence). On the other hand, the convex combination compactness of $T$ implies there exist $\psi_{1}, \psi_{2}, \ldots \in K$ such that $\psi_{j}$ is a convex combination of $\varphi_{j}, \varphi_{j+1} \ldots$ and $T\left(\psi_{j}\right) \rightarrow f$ for some $f \in T(K)$. Since $\psi_{j} \rightarrow \varphi$ we see that $f=T(\varphi)$ by the closed graph property. Thus

$$
\int T\left(\psi_{j}\right) d \mu \rightarrow \int f d \mu=\int T(\varphi) d \mu
$$

On the other hand, $\lim _{j} \int T\left(\psi_{j}\right) d \mu=\lim _{j} \int T\left(\varphi_{j}\right) d \mu$ so $\int T\left(\varphi_{j}\right) \rightarrow \int T(\varphi)$. This shows $L_{\mu}$ is continuous on $K$.

To show that $T(K)$ is convex combination compact, it suffices to prove $T(K)$ is weakly compact (by the Hahn-Banach Theorem). On the other hand, the Dunford-Pettis theorem says that to show that $T(K)$ is weakly compact, it suffices to show that $T(K)$ is uniformly integrable, that is, there exists $\varepsilon_{k} \downarrow 0$ such that

$$
\left|\int_{T(\varphi)<-k} T(\varphi) d \mu\right| \leq \varepsilon_{k} \text { for all } \varphi \in K
$$

But $\mu \in \mathcal{C}$ implies

$$
\int_{0}^{\infty} t \mu\{t<V-\varphi\} d t \leq A \int_{0}^{\infty} t C a p\{t<V-\varphi\} d t \leq C_{1}
$$

Thus $T(K)$ is a bounded subset of $L^{2}(X, d \mu)$ and hence, by Hölder's inequality, $T(K)$ is uniformly integrable. 
The second main difficulty is to prove the Euler-Lagrange equation is satisfied at the point where $F_{\mu}$ reaches its maximum. The problem is that the minimum $\varphi$ may only satisfy $\omega+\frac{i}{2} \partial \bar{\partial} \varphi \geq 0$, and a variation $\varphi_{t}=\varphi+\delta \varphi$ may no longer satisfy this condition even if $\delta \varphi$ is smooth and small. This is overcome using a technique of [BB] which we now describe. Define for each $u$ an upper semi-continuous function on $X$, its $\theta$-psh envelope,

$$
P(u)=\sup \left\{\varphi \in P S H^{1}(X, \theta): \varphi \leq u \text { on } X\right\}
$$

Then $P(u) \in P S H^{1}(X, \theta)$ so the function $g(t)=E(P(\varphi+t \delta \varphi))-L_{\mu}(\varphi+t \delta \varphi)$ is now well-defined for $t \in \mathbf{R}$, and has a maximum at $t=0$. It is shown in [BBGZ] that we still have $0=g^{\prime}(0)=\int_{X} \delta \varphi(M A(\varphi)-d \mu)$. Thus the equation $M A(\varphi)=\mu$ has a solution in the case where $\mu \in \mathcal{C}$.

We now remove the assumption $\mu \in \mathcal{C}$. Thus we let $\mu$ be a non-pluripolar probability measure with finite energy. Our goal is to prove that there exists $\varphi \in \mathcal{T}^{1}$ such that $M A(\varphi)=\mu$. To do this, we make use of the following lemma of Cegrell [Ceg]:

Lemma 21 Let $\mu$ be a non-pluripolar probability measure. Then there exists $\nu \in \mathcal{C}$ and $f \in L^{1}(X, \nu)$ such that $\mu=f \nu$.

Write $\mu=f \nu$ as in the lemma. For $k>0$ choose $\varepsilon_{k} \geq 0$ so that $\mu_{k}=\left(1+\varepsilon_{k}\right) \min (f, k) \nu$ is a probability measure. Then $\mu_{k} \leq 2 k$ Cap so $\mu \in \mathcal{C}$. Thus, by what has been proved thus far, $\mu_{k}=T_{k}^{n}$ for some $T_{k} \in \mathcal{T}^{1}$. Next we observe that $\mu_{k} \leq 2 \mu$. Using the fact that $E^{*}(\mu)<\infty$, one shows that $E^{*}\left(\mu_{k}\right) \leq C<\infty$ for some $C>0$. The bound (11.17) then implies that the $T_{k}$ all lie in the compact set $\left\{J_{0} \leq n C\right\}$. Thus, after passing to a subsequence, we conclude $T_{k} \rightarrow T$ for some $T \in \mathcal{T}^{1}$. Since $T_{k}^{n}=\mu_{k}$ we can take the limit as $k \rightarrow \infty$ to conclude $T^{n}=\mu$ (this follows by Fatou's lemma in the case $n=1$, and a generalization of Fatou's lemma, due to [BEGZ], in the case $n>1$ ).

We remark that if $\mu$ is a non-pluripolar probability measure (not necessarily of finite energy), then one can still apply Lemma 21 to conclude $\mu=f \nu$ and one can still construct $\mu_{k}=\left(1+\varepsilon_{k}\right) \min (f, k) \nu$ as above. Then Theorem 19 implies the existence of $T_{k} \in \mathcal{T}^{1}$ such that $T_{k}^{n}=\mu_{k}$. Since $\mu$ is not assumed to have finite energy, we cannot conclude that the $T_{k}$ lie in a compact subset of $\mathcal{T}^{1}$. On the other hand, we have $T_{k} \subseteq \mathcal{T}^{1} \subseteq \mathcal{T}$ and $\mathcal{T}$ is compact. Thus, after passing to a subsequence, $T_{k} \rightarrow T$ for some $T \in \mathcal{T}$. Then [BBGZ] show that one can again take the limit as $k \rightarrow \infty$ to conclude $T^{n}=\mu$. This gives a variational proof of the following theorem of [BEGZ]:

Theorem 20 Let $\mu$ be a non-pluripolar probability measure on $X$. There there exists $T \in \mathcal{T}(X, \theta)$ such that $T^{n}=\mu$.

We close by describing one more application of the variational method in [BBGZ]. 
Theorem 21 Let $(X, \omega)$ be a manifold of general type and let $\theta=-$ Ricci $(\omega)$. Then there exists $\psi \in P S H^{1}(X, \theta)$ such that

$$
\left(\theta+\frac{i}{2} \partial \bar{\partial} \psi\right)^{n}=e^{\psi} \omega^{n}
$$

To prove this theorem, we consider the functional $F=E-L$ where $L(\varphi)=\frac{1}{2} \log \int_{X} e^{\varphi} \omega^{n}$ and follow the same three steps as in the proof of Theorem 19. The first step is to show that $F$ is $J_{0}$ coercive. The second step is to show that $L$ is continuous (which implies that $F$ is upper semi-continuous). And the third step is to prove that the critical points for $F$ are solutions to $(11.25)$.

Steps one and three proceed exactly as in the proof of Theorem 19. Thus we restrict ourselves to step two, which is much easier than the corresponding step in Theorem 19. Indeed, if $\varphi_{j} \rightarrow \varphi$ is a convergent sequence in $P S H^{1}(X, \theta)$ then after passing to a subsequence, $\varphi_{j} \rightarrow \varphi$ almost everywhere. On the other hand, Hartogs' lemma implies that $\varphi_{j}$ is bounded above. Thus $\int_{X} e^{\varphi_{j}} \omega^{n} \rightarrow \int_{X} e^{\varphi} \omega^{n}$ and hence $L$ is continuous.

We observe that Theorem 21 follows from Theorem 16. To see this, let $\pi: X \rightarrow X_{\text {can }}$ be the canonical model of $X$ and choose $\varphi_{\text {can }}$ to be the solution of (10.8)

$$
\left(\omega+\frac{i}{2} \partial \bar{\partial} \varphi_{\text {can }}\right)^{n}=e^{\varphi_{\text {can }} \Omega .}
$$

where $\frac{i}{2} \partial \bar{\partial} \log \Omega=\omega$. Choose $\Theta$ such that $\frac{i}{2} \partial \bar{\partial} \log \Theta=\theta$. Let $\tilde{\Omega}=\pi^{*} \Omega$ and $\tilde{\varphi}_{\text {can }}=\varphi_{\text {can }} \circ \pi$. Define $\psi$ by the equation $e^{\psi_{\text {can }} \tilde{\Omega}}=e^{\psi} \Theta$. Applying $\pi^{*}$ to both sides of (11.26) we obtain (11.25).

The variational method establishes the existence of generalized solutions to the complex Monge-Ampère equation. It is then important to determine when the generalized solution is actually smooth. One such result is the theorem of Szekelyhidi-Tosatti [ST] which asserts the smoothness of the generalized solution when it is known to be bounded and the right hand side is smooth.

It may also be noteworthy that the above solutions to the Monge-Ampère equation can be obtained as limits of the critical points of certain naturally-defined finite-dimensional analogues of the infinite-dimensional functionals. In the Fano case, the proof makes use of the Moser-Trudinger inequality proved in [PSSW]. Finite-dimensional approximations also play a major role in the construction of solutions to the homogeneous Monge-Ampère equation in $\S 13$.

\section{Uniqueness of Solutions}

It is a remarkable fact that the map $\mathcal{T}^{0}(X, \theta) \rightarrow \mathcal{M}_{X}^{0}$, defined by $T \mapsto T^{n}$, is bijective. The surjectivity is part of Theorem 20. The injectivity was proved in [BEGZ], by adapting 
the proof of Dinew [Di09], who proved the injectivity in the Kähler case. We give here a slightly streamlined version of the proof of Dinew.

Theorem 22 Let $(X, \omega)$ be a compact Kähler manifold and $\mathcal{E}(X, \omega) \subseteq P S H^{1}(X, \omega)$ denote the potentials $\varphi$ such that $\left(\omega+\frac{i}{2} \partial \bar{\partial} \varphi\right)^{n}$ has full mass. Let $\varphi, \psi \in \mathcal{E}(X, \omega)$. Assume $\left(\omega+\frac{i}{2} \partial \bar{\partial} \varphi\right)^{n}=\left(\omega+\frac{i}{2} \partial \bar{\partial} \psi\right)^{n}$. Then $\varphi-\psi$ is constant.

To prove the theorem, we wish to make use of the comparison principle. If we apply it directly to $V=\{\varphi<\psi\}$ we get $\int_{V} \omega_{\psi}^{n} \leq \int_{V} \omega_{\varphi}^{n}$, which is not useful (in fact, the inequality is an equality since $\omega_{\varphi}^{n}=\omega_{\psi}^{n}$ ). Instead, we shall apply the comparison principle to the set $V_{\varepsilon}(\theta, \rho)=\{(1-\varepsilon) \varphi+\varepsilon \theta<(1-\varepsilon) \psi+\varepsilon \rho\}$ where $\theta, \rho$ are potentials to be chosen later. We obtain, for $T$ a positive closed current, and $k \geq 1$,

$$
\int_{V_{\varepsilon}} T \wedge\left((1-\varepsilon) \omega_{\psi}+\varepsilon \omega_{\rho}\right)^{k} \leq \int_{V_{\varepsilon}} T \wedge\left((1-\varepsilon) \omega_{\varphi}+\varepsilon \omega_{\theta}\right)^{k}
$$

Assume $\int_{V_{\varepsilon}} T \wedge \omega_{\psi}^{k}=\int_{V_{\varepsilon}} T \wedge \omega_{\varphi}^{k}$. Then the leading terms cancel so

$$
\int_{V_{\varepsilon}} T \wedge \omega_{\psi}^{k-1} \wedge \omega_{\rho} \leq \int_{V_{\varepsilon}} T \wedge \omega_{\varphi}^{k-1} \wedge \omega_{\theta}+O(\varepsilon)
$$

We shall also need the following generalization of Lemma 7 proved in Dinew [Di09a]:

Theorem 23 Let $\varphi, \psi \in \mathcal{E}(X, \omega)$ and $\mu$ a positive non-pluripolar measure. Assume that $\omega_{\varphi}^{n} \geq f d \mu$ and $\omega_{\psi}^{n} \geq g d \mu$ for some non-negative $f, g \in L^{1}(d \mu)$. Then for $0 \leq k \leq n$

$$
\omega_{\varphi}^{k} \wedge \omega_{\psi}^{n-k} \geq f^{\frac{k}{n}} g^{\frac{n-k}{n}} d \mu
$$

In particular, if $\omega_{\varphi}^{n}=\omega_{\psi}^{n}$, then $\omega_{\varphi}^{n}=\omega_{t \varphi+(1-t) \psi}^{n}$ for all $t \in[0,1]$.

We return to the proof of Theorem 22 and follow the argument in [Di09]. Let $\mu=\omega_{\varphi}^{n}$ and define $f: \mathbf{R} \rightarrow[0,1]$ by $f(t)=\mu(\{\varphi<\psi+t\}$. Then $f$ is left continuous. Moreover, since $f$ is increasing, there is a countable set $\Sigma$ such that $f$ is continuous on $\mathbf{R} \backslash \Sigma$.

The key step in the proof is to show that Image $(f)=\{0,1\}$ : Assume not. Then there exists $\alpha \in \mathbf{R} \backslash \Sigma$ such that $0<f(\alpha)<1$. To see this, let $\beta \in \mathbf{R}$ satisfy $0<f(\beta)<1$. Now choose an increasing sequence $t_{j} \in \mathbf{R} \backslash \Sigma$ such that $t_{j} \rightarrow \beta$. Then we can take $\alpha=t_{j}$ for any sufficiently large $j$. After replacing $\varphi$ by $\varphi-\alpha$ we may assume $\alpha=0$.

Since $0<f(0)<1$ we may choose $0<q<1$ such that $1-q<f(0)<q$. With this choice of $q$ we see that $\mu(\{\varphi<\psi\})<q$ and $\mu(\{\psi<\varphi\})<q$ and $\mu(\{\varphi=\psi\})=0$ (the last equality follows from the fact that $\alpha \in \mathbf{R} \backslash \Sigma)$. To get a contradiction, we consider the probability measure $\hat{\mu}=g \mu$ where $g=\frac{1}{q}$ on $\{\varphi<\psi\}$ and $g=c \mu$ on $\{\varphi \geq \psi\}$ for an appropriately chosen $c>0$. The theorem of Guedj-Zeriahi [GZ] implies there exists 
$\rho \in P S H^{1}(X, \omega)$ with $\sup \rho=0$ and $\omega_{\rho}^{n}=\hat{\mu}$. Then, setting $a=\left(\frac{1}{q}\right)^{1 / n}$, and $\theta=0$, and $k=n$, Theorem 23 implies

$$
\omega_{\psi}^{n-1} \wedge \omega_{\rho} \geq a \omega_{\psi}^{n} \text { on the set } V_{\varepsilon}(\theta, \rho) \subseteq\{\varphi<\psi\}
$$

Substituting in (12.2), and taking the limit, $\varepsilon \downarrow 0$, we get $V_{\varepsilon} \uparrow\{\varphi<\psi\}$ so

$$
a \int_{\varphi<\psi} \omega_{\varphi}^{n} \leq \int_{\varphi<\psi} \omega_{\varphi}^{n-1} \wedge \omega
$$

If instead we take $k=1$ and $T=\omega_{\psi}^{n-1}$ we obtain

$$
a \int_{\varphi<\psi} \omega_{\psi}^{n} \leq \int_{\varphi<\psi} \omega_{\psi}^{n-1} \wedge \omega
$$

Interchanging $\varphi$ and $\psi$ in the second estimate, we get $a \int_{\varphi \geq \psi} \omega_{\varphi}^{n} \leq \int_{\varphi \geq \psi} \omega_{\varphi}^{n-1} \wedge \omega$, where we make use of the fact that $\mu\{\varphi=\psi\}=0$. Adding this to the previous inequality we conclude that $a \leq 1$, a contradiction.

The next step is to show

$$
\int_{v p<\psi} \omega_{\varphi}^{j} \omega_{\psi}^{k-1} \omega^{l+1} \leq \int_{\varphi<\psi} \omega_{\varphi}^{j} \omega_{\psi}^{k} \omega^{l}=0
$$

for all $j, k, l$ such that $j+k+l=n$. To see this, we use induction on $l$. Let $\rho=0$ and $\theta=\varphi$ and $T=\omega_{\varphi}^{j} \omega^{l}$. Then applying (12.2) we obtain, for every $\delta>0$,

$$
\int_{\varphi<(1-\varepsilon) \psi-\delta} \omega_{\varphi}^{j} \omega_{\psi}^{k-1} \omega^{l+1} \leq \int_{\varphi<(1-\varepsilon) \psi-\delta} \omega_{\varphi}^{j} \omega_{\psi}^{k} \omega^{l}+O(\varepsilon)
$$

Taking the limit as $\varepsilon \downarrow 0$ we obtain (12.6) but with $\{\varphi<\psi\}$ replaced by $\{\varphi \leq \psi-\delta\}$. Now take the limit $\delta \downarrow 0$ to obtain (12.6).

Taking $l=n$ we obtain $\int_{\varphi<\psi} \omega^{n}=0$. Similarly $\int_{\varphi>\psi} \omega^{n}=0$. Since these sets are plurifine open, they must be empty. This proves the theorem.

\section{Semiclassical Solutions of Monge-Ampère Equa- tions}

In this section we discuss a particular method for solving Monge-Ampère equations, namely by semiclassical limits of Bergman kernels. The equation accessible this way is the homogeneous complex Monge-Ampère equation. It is a very specific equation, but one which is of great interest in the problem of finding Kähler metrics of constant scalar curvature, and which is intimately linked with the notion of stability in geometric invariant theory (see [D99] and [PS08] for a survey). We shall see how semiclassical limits lead to generalized solutions of this equation. This may be noteworthy in itself from the viewpoint of PDE theory, as generalized solutions of partial differential equations usually arise rather from either variational or Perron methods. 


\subsection{Geodesics in the space of Kähler potentials}

We begin with some geometric motivation. Let $\left(X, \omega_{0}\right)$ be a compact Kähler manifold without boundary of dimension $n$. Then the space $\mathcal{K}$ of Kähler potentials

$$
\mathcal{K}=\left\{\varphi \in C^{\infty}(X) ; \omega_{\varphi} \equiv \omega_{0}+\frac{i}{2} \partial \bar{\partial} \varphi>0\right\}
$$

is formally an infinite-dimensional Riemannian manifold with tangent space $T_{\varphi}(\mathcal{K})=$ $\left\{\delta \varphi \in C^{\infty}(X)\right\}$ and metric

$$
\|\delta \varphi\|^{2}=\int_{X}|\delta \varphi|^{2} \omega_{\varphi}^{n}
$$

The geodesic equation for $\mathcal{K}$ is the Euler-Lagrange equation for the energy functional

$$
\mathcal{E}=\int_{0}^{T} \int_{X} \dot{\varphi}^{2} \omega_{\varphi}^{n} d t
$$

for paths $[0, T) \ni t \rightarrow \varphi(\cdot, t) \in \mathcal{K}$. Under a variation $\delta \varphi$ of this path, we have

$$
\delta \mathcal{E}=\int_{0}^{T} \int_{X}\left(2 \dot{\varphi} \delta \dot{\varphi}+\dot{\varphi}^{2} \Delta_{\varphi} \delta \varphi\right) \omega_{\varphi}^{n} d t
$$

where $\Delta_{\varphi}$ is the Laplacian with respect to the Kähler form $\omega_{\varphi}$. For variations $\delta \varphi$ fixing the end points, integrating by parts gives

$$
\delta \mathcal{E}=-2 \int_{0}^{T} \int_{X} \delta \varphi\left(\ddot{\varphi}-|\nabla \dot{\varphi}|_{\omega_{\varphi}}^{2}\right) \omega_{\varphi}^{n} d t
$$

and thus the geodesic equation for paths $[0, T) \ni t \rightarrow \varphi(\cdot, t) \in \mathcal{K}$ is

$$
\ddot{\varphi}-|\nabla \dot{\varphi}|_{\omega_{\varphi}}^{2}=0
$$

A key observation due to Donaldson [D99] and Semmes [Se] is that this geodesic equation is equivalent to a homogeneous complex Monge-Ampère equation

$$
\left(\pi^{*} \omega_{0}+\frac{i}{2} \partial \bar{\partial} \Phi\right)^{n+1}=0
$$

for the function

$$
\Phi(z, w) \equiv \varphi(z, \log |w|)
$$

on $M \equiv X \times A$, with $A=\left\{w \in \mathbf{C} ; e^{-T}<|w|<1\right\}$, Here $\partial \bar{\partial} \Phi$ is taken with respect to all $(n+1)$ variables $(z, w)$, and $\pi^{*} \omega_{0}$ is the pull-back of $\omega_{0}$ to $M$. Note that $\pi^{*} \omega_{0}$ is not strictly positive, viewed as a $(1,1)$-form on $M$. 
We shall consider both geodesic segments, joining two points $\varphi_{0}$ and $\varphi_{1}$ of $\mathcal{K}$, and geodesic rays, extending from a point $\varphi_{0} \in \mathcal{K}$ to infinity. There is no loss of generality in taking $\varphi_{0}=0$.

The equation for geodesic segments is then a standard Dirichlet problem on the manifold $M=X \times A, \partial A=\left\{w \in \mathbf{C} ;|w|=1\right.$ or $\left.|w|=e^{-T}\right\}$ with $T$ finite, and boundary value $\Phi_{b}$ defined by

$$
\begin{aligned}
& \Phi_{b}(z, w)=0 \text { for }|w|=1 \\
& \Phi_{b}(z, w)=\varphi_{1} \text { for }|w|=e^{-T} .
\end{aligned}
$$

The equation for geodesic rays is more unusual: here $T=\infty$, so the annulus $A$ reduces to the punctured disk $D^{\times}=\{w \in \mathbf{C} ; 0<|w|<1\}$, and $M=X \times D^{\times}$. A boundary value $\Phi_{b}$ is assigned only on the component $|w|=1$ of the boundary of $M$,

$$
\Phi_{b}(z, w)=0 \text { for }|w|=1
$$

but there is no condition near $w=0$. In practice, and with motivation from geometric invariant theory, we shall restrict to the case where $\omega_{0}=c_{1}(L)$, where $L \rightarrow X$ is a positive line bundle, and consider the geodesic rays associated to a test configuration of $L \rightarrow X$. A test configuration (see the precise definition in $\S 13.2$ below) is a one-parameter subgroup (1PS) degeneration of the line bundle $L \rightarrow X$. It produces a limiting singular line bundle, or more precisely a polarized scheme, $L_{0} \rightarrow X_{0}$, which can be viewed as an implicit boundary value for the Dirichlet problem for the homogeneous complex Monge-Ampère equation at $w=0$. The net result is that we shall associate a canonical geodesic ray to each test configuration, starting from an arbitrary point $\varphi_{0} \in \mathcal{K}$. Thus a test configuration can also be viewed as providing a direction where the Cauchy problem admits a generalized solution for infinite time, and hence as a generalized vector field on the space $\mathcal{K}$ of Kähler metrics.

The motivation for test configurations and geodesic rays is the following. It has been shown by Donaldson [D99] and Mabuchi [M87] that $\mathcal{K}$ is a symmetric space with nonpositive curvature. The geodesic rays in $\mathcal{K}$ are then just a generalization of the oneparameter subgroups of finite-dimensional symmetric spaces of negative curvature. In Donaldson's program for the problem of constant scalar curvature metrics [D99], a numerical invariant for geodesic rays can be defined as the limiting value as $t \rightarrow \infty$ of the rate of change of the Mabuchi K-energy along geodesic rays. An infinite-dimensional notion of stability in geometric invariant theory (GIT) can then be defined as the negativity of this numerical invariant of geodesic rays, and we obtain in this way an infinite-dimensional version of the Yau-Tian-Donaldson conjecture, which asserts the equivalence between the existence of a metric of constant scalar curvature in the Kähler class $c_{1}(L)$ and the Kstability of $L \rightarrow X$ in the sense of GIT.

Very recently, Lempert and Vivas [LV] have produced examples of Kähler manifolds $\left(X, \omega_{0}\right)$, specifically tori with certain symmetries, where there are no $C^{3}$ geodesic connecting two given potentials $\varphi_{1}, \varphi_{2} \in \mathcal{K}$. A $C^{\infty}$ geodesic would correspond to a function 
$\Phi(z, w)$ which is $C^{\infty}$ in both $z$ and $w$, a solution of the completely degenerate MongeAmpère equation, and which is strictly plurisubharmonic with respect to $\omega_{0}$ for each $w \in A$. On the other hand, generalized solutions of (13.7) in the sense of pluripotential theory, where $\Phi(z, w)$ is only known to be plurisubharmonic in all variables $(z, w)$, and where $\Phi$ is only of class $C^{1, \alpha}$, have been known for some time. Their existence can be deduced from the general theory of boundary value problems of $\S 9$ [C00, B09b, PS09a], as well as from explicit semi-classical constructions [PS06, PS07, SZ07, SZ10, RZ08]. Other constructions are due to Arezzo-Tian [AT] (by the Cauchy-Kowalevska method), and to Chen [C06], Chen-Sun [CS], Chen-Tang [CT], Ross-Witt Nystrom [RW] and other authors under various types of assumptions. A partial regularity theory for the homogeneous complex Monge-Ampère equation has been proposed by Chen and Tian [CT]. We discuss some of these developments in this section.

\subsection{Geodesics from a priori estimates}

We show how generalized geodesics can be obtained from the existence theorems for the Dirichlet problem of Section $\S 8$. In the present context, $\left(X, \omega_{0}\right)$ is a given compact Kähler manifold without boundary, and the manifolds with boundary of the theorems in Section $\S 8$ are now given by $M=X \times A$, with $A$ an annulus, or $M=X \times D^{\times}$, with $D^{\times}$a punctured disk.

First, we consider the case of geodesic segments, linking $\varphi_{0}=0$ to $\varphi_{1} \in \mathcal{K}$. Here the set-up of the geodesic equation is exactly the same as for Theorem 13, with the difference that the form $\pi^{*} \omega_{0}$ in (13.7) is not strictly positive. However, it is easy to bring ourselves back to the case of a strictly positive form, by constructing a smooth function $\underline{\Phi}$ satisfying

$$
\pi^{*} \omega_{0}+\frac{i}{2} \partial \bar{\partial} \underline{\Phi}>0 \text { on } M, \quad \underline{\Phi}=\Phi_{b} \text { on } \partial M .
$$

Setting then $\Omega_{0}=\pi^{*} \omega_{0}+\frac{i}{2} \partial \bar{\partial} \underline{\Phi}$ and $\Phi=\Psi+\underline{\Phi}$, the equation $\left(\pi^{*} \omega_{0}+\frac{i}{2} \partial \bar{\partial} \Phi\right)^{n+1}=0$ is equivalent to the equation $\left(\Omega_{0}+\frac{i}{2} \partial \bar{\partial} \Psi\right)^{n+1}=0$, with $\Omega_{0}$ now a Kähler form so that Theorem 13 applies at once. The function $\Phi$ is obtained by the following elementary construction (see e.g. Lemma 14 of [PS08]): $\underline{\Phi}=t \varphi_{1}+f(w), t=\log |w|$, with $f(w)$ the solution of the Dirichlet problem $\Delta f(w)=C$ on $A, f=0$ on $\partial A$, and $C$ is a large positive constant. Thus we have established the following theorem, first proved by Chen [C00]:

Theorem 24 Let $\varphi_{0}$ and $\varphi_{1}$ be two points in $\mathcal{K}$. Then there exists a unique generalized geodesic of class $C^{1, \alpha}$, for any $0<\alpha<1$, joining $\varphi_{0}$ and $\varphi_{1}$.

We turn next to geodesic rays. The notion of test configuration alluded to in $\S 13.1$ can be defined precisely as follows (see Donaldson [D02]): 
Definition 3 Let $L \rightarrow X$ be a positive line bundle over a compact complex manifold $X$. A test configuration $\mathcal{T}$ for $L \rightarrow X$ consists of

(1) a scheme $\mathcal{X}$ with a $\mathbf{C}^{\times}$action $\rho$;

(2) a $\mathbf{C}^{\times}$equivariant line bundle $\mathcal{L} \rightarrow \mathcal{X}$, ample on all fibers;

(3) and a flat $\mathbf{C}^{\times}$equivariant map $\pi: \mathcal{X} \rightarrow \mathbf{C}$, where $\mathbf{C}^{\times}$acts on $\mathbf{C}$ by multiplication, with the property that $\left(\pi^{-1}(1), \mathcal{L}_{\left.\right|_{\pi^{-1}(1)}}\right)$ is isomorphic to $\left(X, L^{r}\right)$ for some $r>0$.

We shall also denote $\mathcal{T}$ by

$$
\mathcal{T}=\left(\rho: \mathbf{C}^{\times} \rightarrow \operatorname{Aut}(\mathcal{L} \rightarrow \mathcal{X} \rightarrow \mathbf{C})\right)
$$

where $\operatorname{Aut}(\mathcal{L} \rightarrow \mathcal{X} \rightarrow \mathbf{C})$ is the space of automorphisms of the fibrations $\mathcal{L} \rightarrow \mathcal{X} \rightarrow \mathbf{C}$. A test configuration $\mathcal{L}$ is said to be trivial if $\mathcal{L}=L \times \mathbf{C}$, with the action $\rho(\tau)(\ell, w)=(\ell, \tau w)$, for $(\ell, w) \in L \times \mathbf{C}, \tau \in \mathbf{C}^{\times}$.

It is convenient to introduce the notation $X_{w}=\pi^{-1}(w), L_{w}=\mathcal{L}_{\left.\right|_{\pi^{-1}(w)}}$, and to view $\mathcal{X}$ as $\mathcal{X}=\cup_{w \in \mathbf{C}} X_{w}$. A typical example of a test configuration would be $\mathcal{X}=\cup_{\tau} \sigma_{\tau}(X)$ where $X$ is a submanifold of $\mathbf{C P}^{N}$, and $\sigma_{\tau}=e^{\tau B}$, is a one-parameter subgroup of $G L(N+1)$.

Note that all fibers $\left(X_{w}, L_{w}\right)$ are biholomorphic to $\left(X_{1}, L_{1}\right)$ for $w \neq 0$. However, the "central fiber" $\left(X_{0}, L_{0}\right)$ will usually have singularities. It can be viewed as the limit in the sense of schemes of $\left(X_{w}, L_{w}\right)$ as $w \rightarrow 0$, and it is invariant under the action of $\rho$.

For the construction of geodesic rays, we need the following geometric properties of a test configuration. Let

$$
p: \tilde{\mathcal{X}} \rightarrow \mathcal{X} \rightarrow \mathbf{C}
$$

a resolution of singularities, which can be chosen to be equivariant, in the sense that the homomorphism $\rho$ lifts to a homomorphism $\tilde{\rho}: \mathbf{C}^{\times} \rightarrow \operatorname{Aut}\left(p_{*} \mathcal{L} \rightarrow \mathcal{X} \rightarrow \mathbf{C}\right)$, and that all diagrams commute. Let $\mathcal{X}_{D}=\pi^{-1}(D), \mathcal{X}_{D}^{\times}=\pi^{-1}\left(D^{\times}\right)$, and $\mathcal{L}_{D} \rightarrow \mathcal{X}_{D}=\pi^{-1}(D)$ and $\mathcal{L}_{D}^{\times} \rightarrow \mathcal{X}_{D}^{\times}$be the fibrations above $D$ and $D^{\times}$, with similar definitions for $\tilde{\mathcal{X}}_{D}$, $\tilde{\mathcal{X}}_{D}^{\times}$, and $\tilde{\mathcal{L}}_{D}$ and $\tilde{\mathcal{L}}_{D}^{\times}$. Let the Kähler form $\omega_{0}$ on $X$ be the curvature of a metric $h_{0}$ on $L$. Then an element $\varphi$ of the space of Kähler potentials $\mathcal{K}$ can be identified with a metric $h=h_{0} e^{-\varphi}$ on $L$. We can now state the properties of test configurations which we need:

- There is a biholomorphism

$$
\begin{aligned}
M=X \times D^{\times} & \rightarrow \tilde{\mathcal{X}}^{\times} \\
L \times D^{\times} & \rightarrow \tilde{\mathcal{L}}^{\times}
\end{aligned}
$$

defined by $(\zeta, w) \rightarrow \rho(w)(\zeta) \in L_{w}$, for $\zeta \in L=L_{1}$. If we extend the metric $h_{0}$ on $L$ trivially as a metric on $L \times D^{\times}$, then it can be identified through the above biholomorphism with a metric $H_{0}$ on $\tilde{\mathcal{L}}^{\times}$. The curvature of $H_{0}$ is (the push-forth of) $\Omega_{0}$. If $H$ is any other metric 
on $\tilde{\mathcal{L}}^{\times}$and $\Omega$ its curvature, then $H=H_{0} e^{-\Psi}$, and the equation $\left(\Omega_{0}+\frac{i}{2} \partial \bar{\partial} \Phi\right)^{m}=0$ on $M$ is equivalent to the equation

$$
\left(\Omega+\frac{i}{2} \partial \bar{\partial} \tilde{\Phi}\right)^{n+1}=0 \text { on } \tilde{\mathcal{X}}_{D}^{\times}
$$

with $\tilde{\Phi}=\Phi-\Psi$.

- Recall that $h_{0}$ is any fixed metric on $L$ with positive curvature $\omega_{0}$. It is shown in [PS07a], [PS09b] that there is a metric $H$ on $\tilde{\mathcal{L}}$ (in particular over the whole of $\tilde{\mathcal{X}}_{D}$, including the central fiber), which restricts to $h_{0}$ on $X_{1}$, and which has curvature $\Omega \geq 0$ over $\tilde{\mathcal{X}}$, and $\Omega>0$ on $\tilde{\mathcal{X}}_{D}^{\times}$.

- Furthermore, there exists an effective divisor $E$ supported only in the central fiber of $\tilde{\mathcal{X}}$ and a smooth metric $K$ on $O(E)$ so that

$$
\Omega_{K} \equiv \Omega+\varepsilon \frac{i}{2} \partial \bar{\partial} \log K
$$

is smooth and strictly positive definite everywhere on $\tilde{\mathcal{X}}_{D}$, for all small, strictly positive $\varepsilon$.

We return now to the problem of constructing generalized geodesic rays. Given a test configuration, the above constructions show that a generalized geodesic ray is a solution of the equation $(13.15)$ on $\tilde{\mathcal{X}}_{D}^{\times}$. The above geometric properties of test configurations mean precisely that the hypotheses of Theorem 13.15 are satisfied with $M=\tilde{\mathcal{X}}_{D}$. Note that in this case, the boundary of the manifold is clearly holomorphically flat. Thus we obtain the following theorem [PS09a]:

Theorem 25 Let $L \rightarrow X$ be a positive line bundle over a compact complex manifold $X$. Let $\mathcal{T}$ be any test configuration of $L \rightarrow X$, and let $p$ be an equivariant resolution as in (13.13). Then for any metric $h_{0}$ on $L$ with positive curvature, there is a $C^{1, \alpha}$ generalized geodesic, with bounded potential, starting from $h_{0}$. More precisely, there is a bounded solution $\tilde{\Phi}$ of the equation $\left(\Omega+\frac{i}{2} \partial \bar{\partial} \tilde{\Phi}\right)^{n+1}=0$ on $\tilde{\mathcal{X}}_{D}$, which is of class $C^{1, \alpha}$ on $\tilde{\mathcal{X}}_{D}^{\times}$.

\subsection{Algebraic approximations: the Tian-Yau-Zelditch theorem}

The conjecture of Yau on Kähler-Einstein metrics [Y93], and the Yau-Tian-Donaldson conjecture on the existence of metrics of constant scalar curvature in a Kähler class $c_{1}(L)$ if $L$ is K-stable [D02], are conjectures relating the solvability of a geometric partial differential geometric to a global algebraic condition. One strategy, advocated by Yau and implemented particularly successfully by Donaldson [D01, D10] in the proof of the necessity of stability, has been to approximate transcendental objects such as metrics by their algebraic counterparts, such as Bergman kernels and Fubini-Study metrics. A precise example is the Tian-Yau-Zelditch theorem [Y93, T90a, Z] (also proved independently by Catlin [Cat] and refined by $\mathrm{Lu}[\mathrm{L}]$ ), which can be stated as follows. 
Let $L \rightarrow X$ be a positive line bundle over a compact complex manifold $X$. If $\underline{s}=\left\{s_{\alpha}\right\}_{0}^{N_{k}}$ is a basis for the space $H^{0}\left(X, L^{k}\right)$ of holomorphic sections of $L^{k}, \operatorname{dim} H^{0}\left(X, L^{k}\right)=1+N_{k}$, then the Kodaira map $\iota_{\underline{s}}$ is defined by

$$
\iota_{\underline{s}}: X \ni z \rightarrow\left[s_{0}(z): \cdots: s_{N_{k}}(z)\right] \in \mathbf{C P}^{N_{k}} .
$$

The Kodaira imbedding theorem says that $\iota_{\underline{s}}$ is an imbedding for $k$ sufficiently large. The hyperplane bundle $O(1)$ over $\mathbf{C P}^{N_{k}}$ pulls back to $L^{k}$. The Fubini-Study metric $h_{F S}=$ $\frac{1}{\sum_{\alpha=0}^{N_{k}}\left|s_{\alpha}\right|^{2}}$ on $O(1)$ and $\omega_{F S}=-\frac{i}{2} \partial \bar{\partial} \log h_{F S}$ on $\mathbf{C P}^{N_{k}}$ pull back then to the metrics

$$
\iota_{\underline{s}}^{*}\left(h_{F S}\right)=\frac{1}{\sum_{\alpha=0}^{N_{k}}\left|s_{\alpha}(z)\right|^{2}}, \quad \iota_{\underline{s}}^{*}\left(\omega_{F S}\right)=-\frac{i}{2} \partial \bar{\partial} \log \sum_{\alpha=0}^{N_{k}}\left|s_{\alpha}(z)\right|^{2} .
$$

on $L^{k}$ and $X$ respectively. Note that $h(k) \equiv\left(\iota_{\underline{s}}^{*}\left(h_{F S}\right)\right)^{\frac{1}{k}}$ is then a metric on $L$, and its curvature $\omega(k) \equiv \frac{1}{k} \iota_{\underline{s}}^{*}\left(\omega_{F S}\right)$ is a metric on $X$, which can be viewed as algebraic objects.

Let now $h$ be a metric on $L$ with positive curvature $\omega=-\frac{i}{2} \partial \bar{\partial} \log h>0$. The TianYau-Zelditch theorem asserts that the metric $h$ on $L$ and $\omega$ on $X$ can be approximated asymptotically in $k$ by the metrics $h(k)$ and $\omega(k)$, if the basis $\underline{s}=\left\{s_{\alpha}\right\}_{0}^{N_{k}}$ used to construct the Kodaira imbedding is an orthonormal basis of $H^{0}\left(X, L^{k}\right)$ with respect to the $L^{2}$ metric, $\|s\|^{2} \equiv \int_{X}|s|^{2} h^{k} \omega^{n}$. To see how this comes about, we write

$$
\log \frac{h(k)}{h}=-\frac{1}{k} \log \rho_{k}(z), \quad \omega-\omega(k)=-\frac{i}{2 k} \partial \bar{\partial} \log \rho_{k}(z)
$$

where $\rho_{k}(z)$ is the Bergman kernel (or density of states, since it integrates to $\operatorname{dim} H^{0}\left(X, L^{k}\right)$ ),

$$
\rho_{k}(z) \equiv \sum_{\alpha=0}^{N_{k}}\left|s_{\alpha}(z)\right|^{2} h^{k}(z)
$$

The desired statement follows then from an asymptotic expansion for $\rho_{k}(z)[\mathrm{Z}]$

$$
\rho_{k}(z)=k^{n}\left(1+\sum_{p=1}^{\infty} A_{p}(z) k^{-p}\right)
$$

This expansion is itself a consequence of an asymptotic expansion obtained by Boutet de Monvel and Sjöstrand [BS] for the Szegö kernel on strongly pseudo-convex domains, refining an earlier expansion along the diagonal obtained by Fefferman $[\mathrm{F}]$. The coefficient $A_{1}(z)$ has been shown by $\mathrm{Lu}[\mathrm{L}]$ to be given by $\frac{1}{2} R(z)$, where $R$ is the scalar curvature of $\omega$. This turns out to provide a key link with the problem of constant scalar curvature metrics [D01, D10]. Other generalizations and applications of asymptotic expansions and of the Tian-Yau-Zelditch theorem can be found in [MM, Wg]. 


\subsection{Semi-classical constructions}

Let $\mathcal{K}_{k}$ denote the space $\left\{\left(\iota_{\underline{s}}\left(h_{F S}\right)\right)^{\frac{1}{k}}\right\}$ of pull-backs of the Fubini-Study metrics, under Kodaira imbeddings defined by an arbitrary basis $\underline{s}$ of $H^{0}\left(X, L^{k}\right)$. Since $h_{F S}$ is invariant under $S U\left(N_{k}+1\right)$, we have

$$
\mathcal{K}_{k}=S L\left(N_{k}+1\right) / S U\left(N_{k}+1\right)
$$

Note that the right hand side is a symmetric space with negative curvature. A suggestive consequence of the Tian-Yau-Zelditch theorem is that, in a pointwise sense, we have

$$
\mathcal{K}=\lim _{k \rightarrow \infty} \mathcal{K}_{k}=\lim _{k \rightarrow \infty} S L\left(N_{k}+1\right) / S U\left(N_{k}+1\right)
$$

It is a natural question whether this pointwise approximation can be extended to the approximation of more geometric properties, for example of extended geometric objects. A prime example is whether geodesics in $\mathcal{K}$ can be approximated by geodesics in $\mathcal{K}_{k}$. This translates precisely into whether the solutions of the homogeneous complex Monge-Ampère equations can be approximated by one-parameter subgroups of Bergman kernels [PS06]. We shall see below that the answer is affirmative, see [PS06, PS07, PS09b]. Some refinements of these approximations and their rate of convergence can be found in Berndtsson [Be1, Be2], and in [SZ07, SZ10] in the case of toric varieties. For toric varieties, a similar approximation has been extended to harmonic maps by Rubinstein and Zelditch [RZ08].

We provide now some details. Fix a metric $h_{0}$ on $L$ with positive curvature $\omega_{0}$. Let $\Phi(z, w)$ be a solution of the Dirichlet problem (13.7) with boundary value $\varphi_{0}$ when $|w|=1$. If we view it as either a geodesic segment or a geodesic ray in $\mathcal{K}$ emanating from the corresponding potential $\varphi_{0}$, then this geodesic segment or ray should be the limit of a sequence of one-parameter subgroups in $\mathcal{K}_{k}$, as $k \rightarrow \infty$. Let $B_{k} \in G L\left(N_{k}+1\right)$ be the infinitesimal generator of the one-parameter subgroup in $\mathcal{K}_{k}$, so that the subgroup is given by $w^{B_{k}}, w \in \mathbf{C}^{\times}$. We can assume that $B_{k}$ is diagonal, with eigenvalues $\lambda_{\alpha}^{(k)}, 0 \leq \alpha \leq N_{k}$. Let $\underline{s}=\left\{s_{\alpha}\right\}_{0}^{N_{k}}$ be a basis of $H^{0}\left(X, L^{k}\right)$ which is orthonormal with respect to the $L^{2}$ norm defined by $h_{0}$ and the volume form $\omega_{0}^{n}$. The subgroup $w^{B_{k}}$ acts on the basis $\left\{s_{\alpha}\right\}_{0}^{N_{k}}$ to produce the basis $w^{B_{k}} \cdot \underline{s} \equiv\left\{w^{\lambda_{\alpha}^{(k)}} s_{\alpha}\right\}_{0}^{N_{k}}$. The corresponding pull-backs of the Fubini-Study metrics can be written explicitly as

$$
\Phi_{k}(z, w)=\frac{1}{k} \log \sum_{\alpha=0}^{N_{k}}|w|^{2 \lambda_{\alpha}^{\left(N_{k}\right)}}\left|s_{\alpha}(z)\right|^{2} h_{0}(z)^{k}
$$

Thus the main problem is to choose the appropriate generators $B_{k}$ and to show that the $\Phi_{k}(z, w)$ converge, in a suitable sense, to a solution $\Phi(z, w)$ of the homogeneous complex Monge-Ampère equation. Note that, by the Tian-Yau-Zelditch theorem, the functions $\Phi_{k}(z, w)$ converge to the correct boundary value when $|w|=1$.

We address the problem of choosing the generators $B_{k}$. 
Consider first the case of a generalized geodesic segment joining two points $\varphi_{0}$ and $\varphi_{1}$ in $\mathcal{K}$. There is in this case a natural choice of infinitesimal generator $B_{k}: B_{k}$ is just the matrix of change of bases, from a basis $\left\{s_{\alpha}^{(0)}\right\}_{0}^{N_{k}}$ orthonormal with respect to the $L^{2}$ metric defined by $h_{0}, \omega_{0}^{n}$, to a basis $\left\{s_{\beta}^{(1)}\right\}_{0}^{N_{k}}$ orthonormal with respect to the $L^{2}$ metric defined by $h_{1}, \omega_{1}^{n}$.

Next, consider the case of a test configuration. The group action $\rho(w), w \in \mathbf{C}^{\times}$ preserves the central fiber $\left(X_{0}, L_{0}\right)$. Thus it induces a one-parameter subgroup $w^{B_{k}}$ on the space of holomorphic sections,

$$
w^{B_{k}}: H^{0}\left(X_{0}, L_{0}^{k}\right) \rightarrow H^{0}\left(X_{0}, L_{0}^{k}\right) .
$$

The generators $B_{k}$ are the generators that we are looking for.

Once the generators $B_{k}$, and hence their eigenvalues $\lambda_{\alpha}^{(k)}$ have been chosen, we need a criterion for when the expressions (13.24) have the desired convergence properties. This is provided by the following lemma [PS08]:

Lemma 22 Fix $h_{0} \in \mathcal{K}$ as before. Consider the general Ansatz

$$
\Phi_{k}(z, w)=\left.\frac{1}{k} \log \sum_{\alpha=0}^{N_{k}}|w|^{2 \lambda_{\alpha}^{(k)}}\right|^{2}\left|s_{\alpha}^{(k)}(z)\right|_{h_{0}^{k}}^{2}
$$

where $\left\{s_{\alpha}^{(k)}(z)\right\}$ is an orthonormal basis for $H^{0}\left(X, L^{k}\right)$ with respect to the $L^{2}$ norm defined by $\left(h_{0}^{k}, \omega_{0}^{n}\right)$, and $\lambda_{\alpha}^{(k)}$ are real numbers, $0 \leq \alpha \leq N_{k}$ for each $k$. Then if

(1) There exists a constant $C$ independent of both $\alpha$ and $k$ so that

$$
\left|\lambda_{\alpha}^{(k)}\right| \leq C k
$$

(2) There exists a constant $C_{T}$ independent of $k$ so that

$$
\iint_{X \times\left\{e^{-T}<|w| \leq 1\right\}}\left(\pi^{*} \omega_{0}+\frac{i}{2} \partial \bar{\partial} \Phi_{k}\right)^{n+1} \leq C k^{-1} .
$$

then $\Phi(z, w)=\lim _{k \rightarrow \infty}\left[\sup _{\ell \geq k} \Phi_{k}(z, w)\right]^{*}$ is continuous at $|w|=1$, and satisfies in the sense of pluripotential theory

$$
\left(\pi^{*} \omega_{0}+\frac{i}{2} \partial \bar{\partial} \Phi\right)^{n+1}=0 \text { on } X \times\left\{e^{-T}<|w|<1\right\}, \quad \Phi(z, w)=0 \text { for }|w|=1 .
$$

It can be shown that, in both choices of generators $B_{k}$ for geodesic segments and for geodesic rays, the condition on the uniform growth of the eigenvalues $\lambda_{\alpha}^{(k)}$ is satisfied. The only non-trivial step remaining is to verify the condition on the decay of the masses of the Monge-Ampère measures of $\Phi_{k}(z, w)$. The key observation here is that these masses are essentially cohomological, and given by

$$
\iint_{X \times\left\{e^{-T}<|w|<1\right\}}\left(\pi^{*} \omega_{0}+\frac{i}{2} \partial \bar{\partial} \Phi\right)^{n+1}=\dot{E}(\varphi(\cdot, 0))-\dot{E}(\varphi(\cdot,-T))
$$


where $E(\varphi)$ is the functional (11.1) we had encountered earlier as the anti-derivative of the Monge-Ampère measure $\omega_{\varphi}^{n}$.

We can now state the main theorems of this section and complete their proofs. For geodesic segments, we have [PS06]:

Theorem 26 Let $h_{1} \in \mathcal{K}$ be another metric on $L$ with $\omega_{1}=-\frac{i}{2} \partial \bar{\partial} \log h_{1}>0, M \equiv$ $X \times\left\{e^{-1}<|w|<1\right\}$. Then the generalized solution $\Phi(z, w)$ of the Dirichlet problem (13.7) can be expressed as

$$
\Phi(z, w)=\lim _{k \rightarrow \infty}\left[\sup _{\ell \geq k} \Phi_{\ell}(z, \log |w|)\right]^{*}
$$

where the eigenvalues $\lambda_{\alpha}^{(k)}$ defining $\Phi_{k}(z, w)$ are the eigenvalues of the matrix $B_{k}$ of change of bases from an orthonormal basis of $H^{0}\left(X, L^{k}\right)$ with respect to $h_{0}, \omega_{0}^{n}$ to an orthonormal basis of $H^{0}\left(X, L^{k}\right)$ with respect to $h_{1}, \omega_{1}^{n}$.

Proof: We compute explicitly the right hand side of (13.30). Denote the approximating Fubini-Study metrics in (13.19) for the metrics $h_{0}$ and $h_{1}$ as $h_{0}(k)=e^{-\varphi_{0}(k)} h_{0}$ and $h_{1}(k)=$ $e^{-\varphi_{1}(k)} h_{0}$ respectively. Let $\omega_{a}(k)$ be their curvatures. Let $\left\{s_{a, \alpha}^{(k)}\right\}$ be orthonormal bases with respect to $h_{a}, \omega_{a}^{n}$. Then we have, with $a=0,1$,

$$
\begin{aligned}
\int_{X} \dot{\varphi}_{a}(k) \omega_{a}(k)^{n} & =\frac{2}{k^{n+1}} \int_{X} \sum_{\alpha=0}^{N_{k}} \lambda_{\alpha}^{(k)}\left|s_{a, \alpha}^{(k)}(z)\right|^{2} h_{a}(k)^{k} \omega_{a}(k)^{n} \\
& =\frac{2}{k^{n+1}} \sum_{\alpha=0}^{N_{k}} \lambda_{\alpha}^{(k)}+O\left(\frac{1}{k^{n+2}}\right) N_{k} \max _{\alpha}\left|\lambda_{\alpha}^{(k)}\right|,
\end{aligned}
$$

where we have applied the Tian-Yau-Zelditch theorem. Thus the leading terms cancel in the difference (13.30), giving the desired estimate. Q.E.D.

By the uniqueness of the solution of the Dirichlet problem, this solution must coincide with the solution obtained by Chen [C00] from the method of a priori estimates (see Theorem 24), so it must be $C^{1, \alpha}$. It has also been shown by Berndtsson [Be2] that the convergence described in [PS06] can actually be strengthened to uniform convergence.

For geodesic rays defined by a test configuration, we have [PS07, PS09b]:

Theorem 27 Let $\rho$ be a test configuration for a positive line bundle $L \rightarrow X$ over a compact complex manifold $X$. Let $h_{0}$ be a metric on $L$, with $\omega_{0}=-\frac{i}{2} \partial \bar{\partial} \log h_{0}>0$. Let $\Phi_{k}(z, w)$ be defined by (13.24), where the eigenvalues $\lambda_{\alpha}^{(k)}$ are the eigenvalues of the endomorphisms $B_{k}$ on $H^{0}\left(X_{0}, L_{0}^{k}\right)$ induced by the group action $\rho$. Then

$$
\Phi(z, w)=\lim _{k \rightarrow \infty}\left[\sup _{\ell \geq k} \Phi_{k}(z, w)\right]^{*}
$$

defines a generalized solution of the Dirichlet problem

$$
\left(\pi^{*} \omega_{0}+\frac{i}{2} \partial \bar{\partial} \Phi\right)^{m+1}=0 \text { on } X \times D^{\times}, \quad \Phi(z, w)=0 \text { for }|w|=1 .
$$


The solution is actually of class $C^{1, \alpha}\left(X \times D^{\times}\right)$for any $0<\alpha<1$. It is non-constant when the test configuration is non-trivial.

Proof. As before, it remains only to prove the bound $O\left(k^{-1}\right)$ on the mass of the MongeAmpère measure on $M=X \times D^{\times}$. Consider the functions $\Phi_{k}^{\#}(z, w)$ defined by the same formula as $\Phi_{k}(z, w)$, but with the eigenvalues $\lambda_{\alpha}^{(k)}$ replaced by their traceless counterparts

$$
\lambda_{\alpha}^{\#,(k)}=\lambda_{\alpha}^{(k)}-\frac{\operatorname{Tr} B_{k}}{N_{k}+1} .
$$

Then $\Phi_{k}(z, w)=\Phi_{k}^{\#}(z, w)(z, w)+\frac{\operatorname{Tr} B_{k}}{k\left(N_{k}+1\right)} \log |w|^{2}$. Thus they have the same complex Hessian, and we can evaluate $(13.30)$ with $\Phi_{k}(z, w)$ replaced by $\Phi_{k}^{\#}(z, w)$. The formula (13.30) gives then, with obvious notations,

$$
\int_{X \times D^{\times}}\left(\pi^{*} \omega_{0}+\frac{i}{2} \partial \bar{\partial} \Phi_{k}^{\#}\right)^{n+1}=\lim _{T \rightarrow \infty} \int_{X} \dot{\varphi}(T)^{\#} \omega_{k}(T)^{n}-\int_{X} \dot{\varphi}(0)^{\#} \omega_{k}(0)^{n} .
$$

Since the eigenvalues $\lambda_{\alpha}^{\#(k)}$ sum to 0 , the leading term in the second expression on the right hand side is 0 . As for the first expression, the lemma below shows that it is automatically $O\left(k^{-1}\right)$. This establishes the fact that the limit $\Phi(z, w)$ satisfies the homogeneous complex Monge-Ampère equation.

Lemma 23 Let $\mathcal{T}$ be a test configuration. Then we have

$$
\lim _{T \rightarrow \infty} \int_{X} \dot{\varphi}(T) \omega_{k}(T)^{n}=\frac{1}{k} F
$$

where $F$ is the Donaldson-Futaki invariant of $\mathcal{T}$, defined as the second term $F$ in the following asymptotic expansion

$$
\frac{\operatorname{Tr} B_{k}}{k\left(N_{k}+1\right)}=F_{0}+F k^{-1}+O\left(k^{-2}\right) .
$$

We should say that this lemma was implicit in the paper of Donaldson [D04]. Its explicit statement and proof can be found in [PS07], Mabuchi [M09], and Donaldson [D10].

Unlike in the case of the geodesic segments, the regularity of the geodesic rays obtained from the above theorem does not follow as yet, since the behavior of the ray near $w=0$ has not been addressed. The following lemma gives a complete description of this behavior [PS09b]:

Lemma 24 Let $\mathcal{T}$ be a test configuration, and let $p$ be an equivariant resolution of singularities as considered earlier in $§ 13.2$. Then the function

$$
\Psi_{k} \equiv \Phi_{k}-\Phi_{1}
$$

extends as a smooth function over the whole of $\tilde{\mathcal{X}}_{D}$. Furthermore, it satisfies the following uniform estimate

$$
\sup _{k \geq 1} \sup _{\tilde{\mathcal{X}}_{D}}\left|\Psi_{k}\right| \leq C<\infty
$$


With this lemma, we can show that the function

$$
\Psi=\Phi-\Phi_{1}
$$

is a bounded solution of a homogeneous complex Monge-Ampère equation on $\tilde{\mathcal{X}}_{D}$ with a non-negative background form $\Omega_{1}$. This can be expressed in turn as a homogeneous complex Monge-Ampère equation with a background form $\Omega$ which satisfies all the hypotheses of Theorem 14. Thus the solution must be $C^{1, \alpha}$, and the proof of the theorem is complete.

\subsection{The toric case}

In the toric case, the previous constructions of solutions of homogeneous complex MongeAmpère equations as limits of Bergman metrics can be analyzed more precisely. We can obtain in this manner more detailed information on the approximating paths and their rates of convergence. A remarkable feature also emerges from this study, which is an unexpected relation between the previous semiclassical constructions and the theory of large deviations [SZ07, SZ10].

\subsubsection{Bergman geodesics}

Let $X$ be an $n$-dimensional toric manifold and $L \rightarrow X$ be a positive toric line bundle over $X$. Let $\mathcal{K}_{\mathcal{T}}$ be the space of positively curved smooth toric hermitian metrics on $L$ which are invariant under the compact $\left(S^{1}\right)^{n}$ torus action. Let $h_{0}, h_{1} \in \mathcal{K}_{\mathcal{T}}$ and let $h_{t}$ for $0 \leq t \leq 1$ be the Monge-Ampère geodesic between them as defined in Section $\S 13.4$. We define

$$
\varphi_{t}(z)=\log \left(h_{t}\left(h_{0}\right)^{-1}\right) .
$$

Then $\operatorname{Ricci}\left(h_{t}\right)=\operatorname{Ricci}\left(h_{0}\right)+\frac{i}{2} \partial \bar{\partial} \varphi_{t}$.

The line bundle $L$ is associated to a convex polytope $P$ in $\mathbf{R}^{n}$ which coincides with the image of the moment map by any toric Kähler metric in $c_{1}(L)$. Each integral point $\alpha \in k \bar{P}$ corresponds to a holomorphic section in $H^{0}\left(X, L^{k}\right)$. In particular, $\left\{z^{\alpha}\right\}_{\alpha \in k \bar{P}}$ form a basis for $H^{0}\left(X, L^{k}\right)$ for $z \in\left(\mathbf{C}^{*}\right)^{n}$. Let $\left\{s_{\alpha}(z)\right\}_{\alpha \in k \bar{P} \cap \mathbf{Z}^{n}}$ be an orthornormal toric basis for $H^{0}\left(X, L^{k}\right)$ with respect to the $L^{2}$ norm defined by $\left(h_{0}^{k}, \omega_{0}^{n}\right)$, where $\omega_{0}=-\frac{i}{2} \partial \bar{\partial} \log h_{0}$. Then we define the following paths of Bergman metrics,

$$
h_{t, k}(z)=\sum_{\alpha \in k \bar{P} \cap \mathbf{Z}^{n}} \frac{1}{\left(Q_{h_{0}^{k}}(\alpha)\right)^{1-t}\left(Q_{h_{1}^{k}}(\alpha)\right)^{t}}\left|s_{\alpha}(z)\right|^{2},
$$

where

$$
Q_{h_{0}^{k}}(\alpha)=\left\|s_{\alpha}\right\|_{h_{0}^{k}}^{2}=\int_{X}\left|s_{\alpha}(z)\right|_{h_{0}^{k}}^{2} \omega_{0}^{n}, \quad Q_{h_{1}^{k}}(\alpha)=\left\|s_{\alpha}\right\|_{h_{1}^{k}}^{2}=\int_{X}\left|s_{\alpha}(z)\right|_{h_{1}^{k}}^{2} \omega_{1}^{n} .
$$


The corresponding potentials are given by

$$
\varphi_{k}(t, z)=\frac{1}{k} \log \left(h_{t, k} h_{0}^{-k}\right) .
$$

They correspond to the potentials $\Phi_{k}(z, w)$ of Theorem 26 with $t=\log |w|$.

The following theorem [SZ10] shows that the potentials $\varphi_{k}(t, z)$ actually converge in $C^{2}$ :

Theorem 28 We have

$$
\lim _{k \rightarrow \infty}\left\|\varphi_{k}(t, z)-\varphi_{t}(z)\right\|_{C^{2}([0,1] \times X)}=0
$$

Theorem 28 is a considerable strengthening of both Theorem 26 and Berndtsson's result [Be2] in the toric case. The advantage of studying Bergman metrics on toric manifolds is that toric holomorphic sections are naturally orthogonal to each other and one can analyze the norming constants $Q_{h^{k}}(\alpha)$. On the other hand, toric geodesics $\varphi_{t}$ are alway smooth [Gd]. Thus one may expect a higher order of convergence.

In [RZ08], Theorem 28 is generalized from geodesics of toric Kähler metrics to harmonic maps of a compact Riemannian manifold with boundary into the space of toric Kähler metrics. More precisely, such a harmonic map equation can always be solved, and the solution approximated by harmonic maps into the space of toric Bergman metrics in the $C^{2}$-topology. The case of geodesics corresponds to the case when the Riemannian manifold with smooth boundary is the interval $[0,1]$.

\subsubsection{Geodesic rays and large deviations}

The approximation of the geodesic rays associated in Section $\S 13.4$ to a test configuration $\mathcal{T}$ for a polarization $L \rightarrow X$ can similarly be refined in the case of toric manifolds [SZ07]. An interesting observation is made in [SZ07], which relates the geodesic rays constructed in $§ 13.4$ on toric manifolds to the large deviations principle [V].

Let $L \rightarrow X$ be a very ample toric line bundle over a toric manifold $X$. We use the same notations as in Section \$13.5.1. Let $h=e^{-\varphi}$ a smooth toric hermitian metric on $L$

such that $\omega=\frac{i}{2} \partial \bar{\partial} \varphi \in c_{1}(L)$ is a toric Kähler metric on $X$. On $\left(\mathbf{C}^{n}\right)^{*}$, the potential $\varphi$ can be identified with a smooth convex function $\psi$ on $\mathbf{R}^{n}, \varphi(z)=\psi(\rho)$, with $\rho=\log |z|^{2}$. The Legendre transform of $\psi$ defined by

$$
u(x)=\sup _{\rho \in \mathbf{R}^{n}}(x \cdot \rho-\psi(\rho))
$$

is called the symplectic potential associated to $\psi$. The function $u(x)$ is a smooth convex function on $P$ with appropriate boundary singularities. 
Let $\psi_{t}(\rho)$ be the geodesic ray constructed in Theorem 27 in the toric setting. Then the symplectic potential associated to $\psi_{t}$ is given by

$$
u_{t}(x)=u_{0}(x)-t(R-f(x))
$$

for some positive piecewise linear convex function $f(x)$ on $P$, and $R \in \mathbf{R}$ with $R-f(x)>0$ on $P$. The piecewise function $f(x)$ is an alternative way of describing a test configuration $\mathcal{T}$ in the toric setting [D02].

Define the pair $\left(d \mu_{k}^{\rho}, I^{\rho}(x)\right)$ by

$$
\begin{aligned}
d \mu_{k}^{\rho}(x) & =\left(\Pi_{h^{k}}(z, z)\right)^{-1} \sum_{\alpha \in k \bar{P} \cap \mathbf{Z}^{n}} \frac{\left|s_{\alpha}\right|_{h^{k}}^{2}(z)}{Q_{h^{k}}(\alpha)} \delta_{\frac{\alpha}{k}}(x) \\
I^{\rho}(x) & =u_{0}(x)+\psi_{0}-x \cdot \rho
\end{aligned}
$$

where $\Pi_{h^{k}}(z, z)$ is the Szegö kernel for $\left(L^{k}, h^{k}\right)$ and $\delta_{\alpha / k}(x)$ is a delta function at $\alpha / k$. Then the pair $\left(d \mu_{k}^{\rho}, I^{\rho}(x)\right)$ satisfies the large deviation principle. The measure $d \mu_{k}^{\rho}$ is a probability measure on $\bar{P}$ and the function $I^{\rho}(x)$ is called the rate function associated to $d \mu_{k}^{\rho}$. Varadhan's lemma says that for each $t$ and $\rho$,

$$
\lim _{k \rightarrow \infty} \frac{1}{k} \log \int_{P} e^{k t(R-f(x))} d \mu_{k}^{\rho}(x)=\sup _{x \in P}\left(t(R-f(x))-I^{\rho}(x)\right) .
$$

However, it turns out that

$$
\psi_{t, k}(\rho)-\psi_{0}(\rho)=\frac{1}{k} \log \int_{P} e^{k t(R-f(x))} d \mu_{k}^{\rho}(x)
$$

is exactly the Bergman geodesic ray constructed in Theorem 27, while

$$
\begin{aligned}
\sup _{x \in P}\left(t(R-f(x))-I^{\rho}(x)\right) & =\sup _{x \in P}\left\{x \cdot \rho-\left(u_{0}(x)-t(R-f(x))\right\}-\psi_{0}(\rho)\right. \\
& =\psi_{t}(\rho)-\psi_{0}(\rho) .
\end{aligned}
$$

Therefore Varadhan's lemma immediately gives the pointwise convergence of $\psi_{k, t}$ to $\psi_{t}$. The uniform convergence in $C^{1}(X \times[0,1])$ topology is proved in [SZ07]. The toric geodesic ray $\psi_{t}$ is also shown in $[\mathrm{SZ07}]$ to be $C^{1,1}(X \times[0,1])$, but not $C^{2}(X \times[0,1])$ in general.

\subsubsection{Counter-examples to regularity of higher order than $C^{1,1}$}

It is well-known that solutions of the homogeneous real Monge-Ampère equation may be only of class $C^{1,1}$ and not higher. Such counterexamples have been extended to the complex case by Gamelin and Sibony [GS]. A more general argument for why solutions cannot always be smooth has been given by Donaldson [D]. In this section, we would like to give a simple example which illustrates the fact that, even when geodesic segments may 
be smooth, as in the case of toric varieties, geodesic rays associated to a test configuration may be again at most $C^{1,1}[\mathrm{SZ} 07]$.

Consider the following simple example of a $C^{1,1}$ geodesic ray over $\mathbf{C P}^{1}$. We consider the standard Fubini-Study metric $g_{F S}=\frac{i}{2} \partial \bar{\partial} \varphi_{0}=\frac{i}{2} \partial \bar{\partial} \log \left(1+|z|^{2}\right)=\frac{i}{2} \partial \bar{\partial} \log \left(1+e^{\rho}\right)$ on $\mathbf{C P}^{1}$, where $\varphi_{0}=\log \left(1+e^{\rho}\right)$ and $\rho=\log |z|^{2}$. Then the moment map can be constructed by

$$
x=\frac{\partial \log \left(1+e^{\rho}\right)}{\partial \rho}=\frac{e^{\rho}}{1+e^{\rho}} \in(0,1) .
$$

The symplectic potential $u_{0}$ corresponding to $\varphi_{0}$ is given by

$$
u_{0}(x)=x \rho-\varphi_{0}(\rho)=x \log x+(1-x) \log (1-x), \quad x=\frac{e^{\rho}}{1+e^{\rho}} .
$$

Let $f(x)=|x-1 / 2|$ be a piecewise linear convex function. Then

$$
u_{t}(x)=u_{0}(x)+t f(x)=x \log x+(1-x) \log (1-x)+t|x-1 / 2|
$$

induces a geodesic ray. Now we can calculate the Kähler potential $\varphi_{t}(\rho)$ corresponding to $u_{t}$.

By applying the Legendre transform to $u_{t}$, we can show by a straightforward calculations that

$$
\varphi_{t}(\rho)=\rho x-u_{t}(x), \quad \rho=\frac{\partial u_{t}}{\partial x}
$$

and

$$
\varphi_{t}(\rho)= \begin{cases}-\frac{t}{2}+\log \left(1+e^{\rho+t}\right), & \rho \in(-\infty,-t) \\ \frac{\rho}{2}+\log 2, & \rho \in(-t, t) \\ \frac{t}{2}+\log \left(1+e^{\rho-t}\right), & \rho \in(t, \infty)\end{cases}
$$

Let $\varphi(t, z)=\varphi_{t}(\rho)-\varphi_{0}$. Then $\varphi$ is an $\omega_{F S^{-}}$psh function in $C^{1,1}(\mathbf{R} \times X)$, but it is not of class $C^{2}(\mathbf{R} \times X)$.

\subsection{The Cauchy problem for the homogeneous Monge-Ampère euation}

The construction of geodesic rays associated to a test configuration can be viewed as the solution of the Cauchy problem for the homogeneous complex Monge-Ampère equation, with the initial velocity provided, implicitly, by the test configuration. Even though the Cauchy problem is not well-posed in the sense of Hadamard, it is instructive to examine when there are solutions and when and how they break down. Such an analysis has been provided recently by Rubinstein and Zelditch [RZ10a] for convex solutions of the real Monge-Ampère equation. 
Consider the Cauchy problem

$$
\begin{aligned}
& \operatorname{det}\left(\nabla^{2} \varphi\right)=0 \text { on }[0, T] \times \mathbf{R}^{n} \\
& \varphi(0, x)=\varphi_{0}(x) \text { on } \mathbf{R}^{n}, \quad \frac{\partial \varphi}{\partial t}(0, x)=\psi_{0}(x) \text { on } \mathbf{R}^{n} .
\end{aligned}
$$

It is shown in [RZ10b] that the above equation can be solved via Legendre transform until the Legendre transform of the solution stops being convex. More precisely, the Legendre transform of $\varphi_{t}$ is given by

$$
u_{t}=u_{0}+t v
$$

The function $u_{t}$ will stop being convex at a certain $T_{\text {span }}>0$ if $v$ is not convex, thus $\psi_{t}$ stops solving the HRMA after $T_{\text {span }}$. A candidate solution is constructed in [RZ10b] for $t \geq T_{\text {span }}$, however, it does not solve the homogeneous real Monge-Ampère equation even in a weak sense and it is not differentiable in general. Before $T_{\text {span }}$, the solution can also be approximated by Toeplitz quantization of the Hamiltonian flow defined by the Cauchy data [RZ10a].

\section{Envelopes and the Perron Method}

The complex Monge-Ampère equation satisfies the comparison principle. Thanks to this, generalized solutions can be obtained by the Perron method, as envelopes of families of plurisubharmonic functions. In this section, we describe some results obtained in this manner, focusing on the homogeneous case.

\subsection{Envelopes}

The Perron method for complex Monge-Ampère equations was first developed by Bedford and Taylor [BT76] for degenerate complex Monge-Ampère equations on bounded domains in $\mathbf{C}^{n}$. A special case of their results of particular interest to our considerations is the following. Let $D \subset \mathbf{C}^{n}$ be a smooth, bounded strictly pseudoconvex domain in $\mathbf{C}^{n}$, and let $f \in C(\partial D)$. Define the following family of plurisubharmonic functions

$$
\mathcal{E}_{D, f}=\left\{u \in P S H(D)|u|_{\partial D} \leq f\right\}
$$

and its upper envelope

$$
\hat{u}(z)=\sup _{\mathcal{E}_{D, f}} u(z)
$$

Then $\hat{u} \in \mathcal{E}_{D, f} \cap C(\bar{D}), u$ is of class $C^{1,1}$ in the interior of $D$, and $u$ is the unique solution of the Dirichlet problem for the homogeneous complex Monge-Ampère equation

$$
\left(\frac{i}{2} \partial \bar{\partial} u\right)^{n}=0 \text { on } D,\left.\quad u\right|_{\partial D}=f \text { on } \partial D .
$$


The local $C^{1,1}$ regularity follows from the $C^{1,1}$ regularity of the solution of the Dirichlet problem for the unit ball [BT76].

The Perron method has been widely applied since for the complex Monge-Ampère equations, and there has been considerable progress, thanks partly to the infusion of new techniques and improved approximation theorems for plurisubharmonic functions. In particular, the following results were obtained relatively recently by Berman and Demailly for certain homogeneous complex Monge-Ampère equations, generalizing the geodesic equations considered in $\S 13$.

Let $\left(X, \omega_{X}\right)$ be an $n$-dimensional compact Kähler manifold with a smooth Kähler form $\omega_{X}$. Let $\Sigma$ be a strictly pseudoconvex domain in $\mathbf{C}^{m}$ with $\rho$ being a smooth strictly plurisubharmonic defining function for $\Sigma$ and $\Sigma=\{\rho<0\}$. We consider the product manifold $M=X \times \Sigma$ and let $\omega_{M}=\omega_{X}+\omega_{\Sigma}$ be a Kähler form on $M$, where $\omega_{\Sigma}=\frac{i}{2} \partial \bar{\partial} \rho$. Let $\pi_{X}: M \rightarrow X$ and $\pi_{\Sigma}: M \rightarrow \Sigma$ be the natural projection maps.

Let $\alpha$ be closed real $(1,1)$ form on $M$ with bounded coefficients, with $\left.\alpha_{s} \equiv \alpha\right|_{\{s\} \times X} \geq$ $\varepsilon \omega_{X}$ for some $\varepsilon>0$ and all $s \in \Sigma$. Let $f$ be a continuous function on $\bar{M}$ such that for each $s \in \partial \Sigma,\left.f\right|_{X_{s}} \in P S H\left(X, \alpha_{s}\right)$, where $X_{s}=\pi_{X}^{-1}(s)$. We define

$$
\mathcal{E}_{M, \alpha, f}=\{u \mid u \in P S H(M, \alpha) \cap C(M), u \leq f \text { on } \partial M\}
$$

and the upper envelope of $\mathcal{E}_{M, \alpha, f}$ by

$$
\varphi=\sup _{\mathcal{E}_{M, \alpha, f}} u
$$

The following theorem was proved in $[\mathrm{BD}]$;

Theorem 29 Let $\left(X, \omega_{X}\right), \Sigma, \alpha$, and $f \in C(\partial M)$ satisfy all the properties listed above, and define the family $\mathcal{E}_{M, \alpha, f}$ and its upper envelope $\varphi$ as above. Then the function $\varphi$ is the unique $\alpha$-plurisubharmonic solution of the Dirichlet problem

$$
\left(\alpha+\frac{i}{2} \partial \bar{\partial} \varphi\right)^{\operatorname{dim} M}=0 \text { on } M, \quad \varphi=f \quad \text { on } \partial M
$$

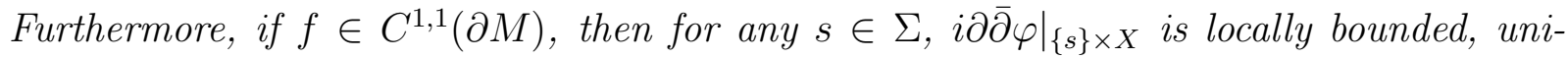
formly in $s \in \Sigma$.

The proof in [BD] depends on the Kiselman infimum principle and refined regularization techniques for plurisubharmonic functions. Here we discuss only the special case when $\alpha=\omega_{X}$ (more precisely, the pull-back of $\omega_{X}$ to $M$ ) and $f \in C^{\infty}(\partial M)$. In this case, the fiber-wise regularity can be obtained by the method of elliptic regularization used earlier for the geodesic equation and the standard $C^{2}$ estimates of Yau, as described in $\S 6$. 
First we show that $\varphi$ is the solution of the Dirichlet problem. Let $\omega_{X, f}=\omega_{X}+\frac{i}{2} \partial \bar{\partial} f$ after extending $f$ to a smooth function on $\bar{M}$. The original problem is equivalent to the same problem formulated rather with

$$
\mathcal{E}_{M, \omega_{X, f}, 0}=\left\{u \mid u \in P S H\left(M, \omega_{X, f}\right), u \leq 0 \text { on } \partial M\right\}, \quad \varphi=\sup _{\mathcal{E}_{M, \omega_{X, f}, 0}} u .
$$

We show that $\varphi$ is continuous on $\partial M$. The form $\omega_{X, f}+A \frac{i}{2} \partial \bar{\partial} \rho$ is a Kähler form on $M$ for sufficiently large $A>0$. Hence $A \rho \in \mathcal{E}_{M, \omega_{X, f}, 0}$ and then $A \rho \leq \varphi$. On the other hand, for any $u \in \mathcal{E}_{M, \omega_{X, f}, 0}, u+A \rho$ is plurisubharmonic on $\pi_{X}^{-1}(z)$ for each $z \in X$, for sufficiently large $A>0$ independent of the choice $z \in X$. Thus $u+A \rho \leq 0$ since $u+A \rho \leq 0$ on $\partial\left(\pi_{X}^{-1}(z)\right)$. It easily follows that

$$
B \rho \leq \varphi \leq-B \rho
$$

for some $B>0$. In particular, $\varphi$ is continuous on $\partial M$.

Next we show that $\varphi$ is continuous in $M$. First we fix $A>0$ with

$$
A \rho \leq \varphi \leq-A \rho
$$

For any compact subset $K$ in $M$ and any sufficiently small $\epsilon>0$, we choose $\delta=(4 A)^{-1} \epsilon$ so that $K \subset M_{4 \delta}$ and $\varphi \leq \epsilon / 4$ on $M \backslash M_{4 \delta}$, where

$$
M_{\delta}=\{(z, s) \in M \mid \rho(z, s)<-\delta\}
$$

By Demailly's regularization techniques [D], there exists a decreasing sequence $\left\{u_{j}\right\} \subset$ $P S H\left(M,\left(\frac{j+1}{j}\right) \omega_{X, f}\right) \cap C\left(M_{\delta / 2}\right)$ which converges to the $\varphi^{*}$, the upper semi-continuous envelope of $\varphi$. We define

$$
\tilde{u}_{j}(z, s)=\left\{\begin{array}{cc}
\max \left(\frac{j}{j+1} u_{j}-\epsilon, 2 A \rho\right), & (z, s) \in M_{\delta} \\
2 A \rho & (z, s) \notin M_{\delta} .
\end{array}\right.
$$

On $\partial M_{\delta}, \frac{j}{j+1} u_{j}-\epsilon \leq-\epsilon / 2 \leq-2 A \delta$. Hence $\tilde{u}_{j} \in \mathcal{E}_{M, \omega_{X, f}, 0}$. Furthermore, on $K$,

$$
\frac{j}{j+1} u_{j}-\epsilon \geq \frac{j}{j+1} \varphi-\epsilon \geq \frac{j}{j+1} A \rho-4 A \delta \geq A \rho+A \rho=2 A \rho,
$$

and so $\tilde{u}_{j}=\frac{j}{j+1} u_{j}-\epsilon$. It follows immediately that

$$
\varphi^{*} \leq u_{j}=\frac{j+1}{j}\left(\tilde{u}_{j}+\epsilon\right) \leq \frac{j+1}{j} \varphi+\frac{j+1}{j} \epsilon
$$

or $0 \leq u_{j}-\varphi \leq \frac{1}{j}|\varphi|+\epsilon$. Therefore $u_{j}$ converges to $\varphi$ uniformly in $L^{\infty}(K)$ and so $\varphi^{*}$ is continuous in $K$. In conclusion, $\varphi=\varphi^{*} \in \mathcal{E}_{M, \omega_{X, f}, 0} \cap C(\bar{M})$. 
Finally, we show that $\left(\frac{i}{2} \partial \bar{\partial} \hat{u}\right)^{\operatorname{dim} M}=0$. Fix any Euclidean ball $B$ in $M$. There exists $\eta \in C^{\infty}(B)$ such that $\omega_{X, f}=\frac{i}{2} \partial \bar{\partial} \eta$ on $B$. Let

$$
\mathcal{E}_{B,\left.(\varphi+\eta)\right|_{\partial B}}=\left\{v|\varphi \in P S H(B), v|_{\partial B}=\left.(\varphi+\eta)\right|_{\partial B}\right\}
$$

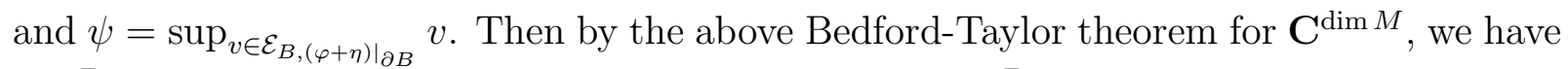
$\left(\frac{i}{2} \partial \bar{\partial} \psi\right)^{\operatorname{dim} M}=0$ and $\varphi=\psi-\eta$ on $\bar{B}$. Hence $\left(\omega_{X, f}+\frac{i}{2} \partial \bar{\partial} \varphi\right)^{\operatorname{dim} M}=0$ on $B$.

We turn to the proof of fiber wise regularity. Consider the following elliptic regularization of the homogeneous Monge-Ampère equation,

$$
\left(\omega_{X}+\frac{i}{2} \partial \bar{\partial} \varphi_{\varepsilon}\right)^{n+m}=\varepsilon \omega_{M}^{n+m} \text { on } M, \quad \varphi_{\epsilon}=f \text { on } \partial M
$$

For any $\varepsilon>0$, there exists a unique smooth solution $\varphi_{\epsilon}$ in $\bar{M}$. In fact, since $\left(\omega_{X}+\right.$ $\left.\frac{i}{2} \partial \bar{\partial} \underline{u}_{s}\right)^{\operatorname{dim} M}>\epsilon \omega_{M}^{\operatorname{dim} M}$, the function $\underline{u}_{s} \equiv f+A \rho$ is a subsolution satisfying $\left.\underline{u}_{s}\right|_{\partial M}=\left.f\right|_{\partial M}$ for sufficiently large $A>0$. Thus $\varphi_{\varepsilon} \geq \underline{u}_{\varepsilon}$. Furthermore $\varphi_{\varepsilon} \leq \varphi \in \mathcal{E}_{M, \omega_{X}, f}$, and we have

$$
\left\|\varphi_{\varepsilon}\right\|_{C^{0}(M)} \leq C
$$

uniformly in $\varepsilon$.

It suffices now to show that there exists $C>0$ such that for any $\epsilon \in(0,1)$ and $s \in \bar{\Sigma}$,

$$
\left.\left(\omega_{X}+\frac{i}{2} \partial \bar{\partial} \varphi_{\varepsilon}\right)\right|_{X_{s}} \leq\left. C \omega_{X}\right|_{X_{s}}
$$

It would follow then that, for any $s \in \bar{\Sigma}$,

$$
\left.\left(\omega_{X}+\frac{i}{2} \partial \bar{\partial} \varphi\right)\right|_{X_{s}} \leq C \omega_{X}
$$

since $\varphi_{\epsilon}$ is increasingly monotone as $\varepsilon \rightarrow 0$ and $\varphi_{\varepsilon}$ converges to $\varphi$ uniformly.

To establish (14.17), let $\omega_{\varepsilon}=\omega_{X}+\frac{i}{2} \partial \bar{\partial} \varphi_{\varepsilon}$. We denote $g_{X}, g_{\Sigma}, g_{M}$ and $g_{\varepsilon}$ be the Kähler metrics associated to $\omega_{X}, \omega_{\Sigma}, \omega_{M}$ and $\omega_{\varepsilon}$. We always use the product coordinates for $M$, where $(z, s)=\left(z_{1}, \ldots, z_{n}, s_{1}, \ldots, s_{m}\right)$ and $z \in X$ and write $\omega_{M}=\frac{i}{2}\left(g_{X}\right)_{\bar{j} i} d z^{i} \wedge d \bar{z}^{j}+$ $\frac{i}{2}\left(g_{\Sigma}\right)_{\bar{\beta} \alpha} d s^{\alpha} \wedge d \bar{s}^{\beta}$.

We define

$$
H_{\epsilon}=\frac{\left(\omega_{X}+\frac{i}{2} \partial \bar{\partial} \varphi_{\varepsilon}\right) \wedge \omega_{X}^{n-1} \wedge \omega_{\Sigma}^{m}}{\omega_{X}^{n} \wedge \omega_{\Sigma}^{m}}
$$

Notice that $H_{\varepsilon}$ is the trace of the relative endomorphism between $\left.\omega_{\varepsilon}\right|_{X_{s}}$ and $\omega_{X}$,

$$
H_{\varepsilon}(z, s)=\operatorname{Tr}_{\omega_{X}}\left(\left.\omega_{\epsilon}\right|_{X_{s}}\right)(z)=\sum_{i, j=1}^{n}\left(g_{X}\right)^{i \bar{j}}\left(g_{\varepsilon}\right)_{\bar{j} i}
$$


where $g_{X}$ is the Kähler metric associated to $\omega_{X}$ and $g_{\varepsilon}$ is the Kähler metric associated to $\omega_{X}+\frac{i}{2} \partial \bar{\partial} \varphi_{\varepsilon}$. The same calculations as in Yau's Schwarz lemma [Y78a] or in Yau's second order estimates, see $\S 6$, show that there exists $C_{1}>0$ such that for all $\varepsilon \in(0,1)$, we have on $M$,

$$
\Delta_{\varepsilon} \log H_{\varepsilon} \geq-C_{1} \operatorname{Tr}_{\omega_{\varepsilon}}\left(\omega_{M}\right)-C_{1}
$$

where $\Delta_{\varepsilon}$ is the Laplacian operator on $M$ with respect to $\omega_{\varepsilon}$. We also have

$$
\Delta_{\varepsilon}\left(-\varphi_{\varepsilon}+\rho\right)=-(n+m)+\operatorname{Tr}_{\omega_{\varepsilon}}\left(\omega_{X}+\omega_{\Sigma}\right)=-(n+m)+\operatorname{Tr}_{\omega_{\varepsilon}}\left(\omega_{M}\right) .
$$

Then there exist $C_{2}, C_{3}, C_{4}>0$ which are independent of $\epsilon \in(0,1)$ such that

$$
\begin{aligned}
\Delta_{\varepsilon}\left(\log H_{\varepsilon}-A \varphi_{\varepsilon}+A \rho\right) & \geq\left(A-C_{1}\right) \operatorname{Tr}_{\omega_{\varepsilon}}\left(\omega_{M}\right)-C_{1}-A(n+m) \\
& \geq C_{2}\left(\operatorname{Tr}_{\omega_{M}}\left(\omega_{\varepsilon}\right)\right)^{1 /(n+m-1)}\left(\frac{\omega_{M}^{n+m}}{\omega_{\varepsilon}^{n+m}}\right)^{1 /(n+m-1)}-C_{2} \\
& \geq C_{3} H_{\varepsilon}-C_{4} .
\end{aligned}
$$

On the other hand,

$$
\left.H_{\varepsilon}\right|_{\partial M}=\left.\frac{\left(\omega_{X}+\frac{i}{2} \partial \bar{\partial} f\right) \wedge \omega_{X}^{n-1}}{\omega_{X}^{n}}\right|_{\partial M}
$$

is uniformly bounded from above for all $\varepsilon \in(0,1)$. Applying the maximum principle, we obtain a constant $C_{5}>0$ such that for all $\varepsilon \in(0,1)$,

$$
H_{\epsilon} \leq C_{5}
$$

since both $u_{\varepsilon}$ and $\rho$ are uniformly bounded in $C(\bar{M})$. The proof of the fiberwise regularity is complete.

\subsection{Envelopes with integral conditions}

In the previous section, we have seen how envelopes with pointwise Dirichlet conditions can produce solutions to the Dirichlet problem for complex Monge-Ampère equation. It would be interesting to determine whether envelopes with integral conditions can be effectively used to produce other solutions of Monge-Ampère equations, or other canonical metrics. We describe some examples of such envelopes below.

One example is the following hermitian metric defined by Tsuji [Ts07] on projective manifolds of general type, generalizing the metric introduced in [NS]. Let $X$ be a smooth projective variety of general type. Fix a smooth hermitian metric $h_{0}$ on $K_{X}$ and define

$$
\varphi_{\text {can }}(z)=\sup \left\{\varphi(z) \mid \operatorname{Ric}\left(h_{0}\right)+\frac{i}{2} \partial \bar{\partial} \varphi \geq 0, \quad \int_{X} e^{\varphi} h_{0}^{-1}=1\right\}
$$


and

$$
h_{c a n}=e^{-\varphi_{c a n}} h_{0} .
$$

It has been shown by Berman and Demailly [BD] that this metric $h_{\text {can }}$ coincides with the metric $\tilde{h}_{\text {can }}$ defined instead by

$$
\tilde{h}_{c a n}(z)=\inf _{m \in \mathbf{Z}^{+}} \inf \left\{\left.(\sigma \wedge \bar{\sigma}(z))^{-1 / m}\left|\int_{X}\right| \sigma \wedge \bar{\sigma}\right|^{1 / m}=1, \sigma \in H^{0}\left(X, m K_{X}\right)\right\} .
$$

which is manifestly a birational invariant, $\tilde{h}_{c a n}$ is a birational invariant since $H^{0}\left(X, m K_{X}\right)$ is invariant under birational transformations.

Another example is the following. Let $\Omega$ be a bounded strictly pseudoconvex domain in $\mathbf{C}^{n}$. We define

$$
\varphi_{\text {can }}(z)=\sup \left\{\varphi(z) \mid \frac{i}{2} \partial \bar{\partial} \varphi \geq 0, \quad \int_{\Omega} e^{\varphi}\left(\frac{i}{2} \partial \bar{\partial}|z|^{2}\right)^{n}=1\right\} .
$$

We also define $\left(h_{\text {can }}\right)^{-1}=e^{\varphi_{\text {can }}}\left(\frac{i}{2} \partial \bar{\partial}|z|^{2}\right)^{n}$ to be the canonical measure on $\Omega$.

Lemma $25 \varphi_{\text {can }}$ is a plurisubharmonic function on $\Omega$.

Proof. First, we show that $\varphi_{\text {can }}$ is bounded from above in any compact subset of $\Omega$. Suppose not, then by taking a subsequence, there exist a sequence of points $z_{j} \rightarrow \hat{z} \in \Omega$ and a sequence of psh functions $\varphi_{j}$ with $\int_{\Omega} e^{\varphi_{j}}=1$ such that

$$
\varphi_{j}\left(z_{j}\right) \rightarrow \infty
$$

Without loss of generality, we can assume that $B\left(z_{j}, r\right) \subset \subset \Omega$ for all $j$ for some fixed $r>0$. Then by the mean value inequality and Jensen's inequality, there exist positive constants $C_{1}, C_{2}, C_{3}$ independent on $j$ such that

$$
1=\int_{\Omega} e^{\varphi_{j}} \geq C_{1} e^{\int_{\Omega} \varphi_{j}} \geq C_{2} e^{\int_{B\left(z_{j}, r\right)} \varphi_{j}} \geq C_{3} e^{\varphi_{j}\left(z_{j}\right)} \rightarrow \infty
$$

This is a contradiction.

Next we have to show that $\varphi_{\text {can }}=\left(\varphi_{\text {can }}\right)^{*}$. By the definition of $\varphi_{\text {can }}$, for any $\hat{z} \in \Omega$, there exists a sequence $z_{j} \rightarrow \hat{z}$ and psh $\varphi_{j}$ with $\int_{\Omega} e^{\varphi_{j}}=1$ such that

$$
\varphi_{j}\left(z_{j}\right) \rightarrow\left(\varphi_{\text {can }}\right)^{*}(\hat{z})
$$

By taking a subsequence, we can assume that $\varphi_{j}$ converges to a psh function $\varphi$ in $L^{1}(\Omega)$ and thus almost everywhere. In particular, $\int_{\Omega} e^{\varphi} \leq 1$ by Fatou's lemma. On the other hand,

$$
\begin{aligned}
\varphi_{\text {can }}(\hat{z}) & \geq \varphi(\hat{z})=\lim _{r \rightarrow 0} \frac{1}{\operatorname{vol}(B(\hat{z}, r))} \int_{B(\hat{z}, r)} \varphi=\lim _{r \rightarrow 0} \lim _{j \rightarrow \infty} \frac{1}{\operatorname{vol}\left(B\left(z_{j}, r\right)\right)} \int_{B\left(z_{j}, r\right)} \varphi_{j} \\
& \geq \lim _{j \rightarrow \infty} \varphi_{j}\left(z_{j}\right)=\left(\varphi_{\text {can }}\right)^{*}(\hat{z}) .
\end{aligned}
$$

The lemma is proved. Q.E.D.

The following theorem provides a more algebraic characterization of $\varphi_{\text {can }}$ : 
Theorem $30 \varphi_{\text {can }} \in P S H(\Omega) \cap C(\Omega)$ and

$$
\varphi_{\text {can }}(z)=\sup _{m \in \mathbf{Z}^{+}} \sup \left\{\left.\frac{1}{m} \log |f|^{2}(z)\left|f \in \mathcal{O}(\Omega), \quad \int_{\Omega}\right| f\right|^{\frac{2}{m}}\left(\frac{i}{2} \partial \bar{\partial}|z|^{2}\right)^{n}=1\right\} .
$$

Proof. Denote by $\varphi_{\text {can,alg }}$ the right-hand side of (14.31). It is easy to see that $\varphi_{\text {can,alg }} \leq$ $\varphi_{\text {can }}$, so it suffices to show that $\varphi_{\text {can alg }} \geq \varphi_{\text {can }}$.

By the Ohsawa-Takegoshi extension theorem, for any psh $\varphi$ on $\Omega$ with $\int_{\Omega} e^{\varphi}=1$ and any point $z \in \Omega$, there exists a holomorphic function $f$ on $\Omega$ such that for any $m \in \mathbf{Z}^{+}$,

$$
|f|^{2} e^{-(m-1) \varphi}(z)=1 \text { and } \int_{\Omega}|f|^{2} e^{-(m-1) \varphi} \leq C,
$$

where $C$ does not depend on $\varphi$ or $m$. By Hölder's inequality, we have

$$
\int_{\Omega}|f|^{2 / m} \leq\left(\int_{\Omega}|f|^{2} e^{-(m-1) \varphi}\right)^{1 / m}\left(\int_{\Omega} e^{\varphi}\right)^{(m-1) / m} \leq(C)^{1 / m}
$$

Let $F=\frac{f}{\left(\int_{\Omega}|f|^{2 / m}\right)^{m}}$. Then $F \in \mathcal{O}(\Omega)$ with $\int_{\Omega}|F|^{2 / m}=1$, and

$$
\frac{1}{m} \log |F|^{2}(z) \geq(m-1) \varphi(z)-\frac{1}{m} C=\varphi(z)-\frac{1}{m}(\varphi(z)+C) .
$$

For fixed $z, \varphi(z)$ and $C$ are uniformly bounded from below. By letting $m \rightarrow \infty$, we get

$$
\varphi_{\text {can, alg }}(z) \geq \varphi(z) .
$$

Since this is true for any $z$ and any psh $\varphi$ with $\int_{\Omega} e^{\varphi}=1$, we have $\varphi_{\text {can,alg }}(z) \geq \varphi_{\text {can }}(z)$, and hence $\varphi_{\text {can,alg }}=\varphi_{\text {can }}$.

Now we can show that $\varphi_{\text {can }}$ is continuous. It suffices to show that $\varphi_{\text {can }}$ is lower semicontinuous. Suppose not. Then there exists $\hat{z} \in \Omega$ and $\varepsilon>0$ and a sequence of points $z_{j} \in \Omega$ converging to $\hat{z}$ so that

$$
\varphi_{\text {can }}\left(z_{j}\right)<\varphi_{\text {can }}(\hat{z})-\epsilon .
$$

Also there exist $f \in \mathcal{O}(\Omega)$ and $m \in \mathbf{Z}^{+}$such that

$$
\int_{\Omega}|f|^{2 / m}=1, \quad \frac{1}{m} \log |f|^{2}(\hat{z})>\varphi_{\text {can }}(\hat{z})-\frac{\epsilon}{2} .
$$

Then there exist $r>0$ such that for all $z \in B(\hat{z}, r)$,

$$
\varphi_{\text {can }}(z) \geq \frac{1}{m} \log |f|^{2}(\hat{z})>\varphi_{\text {can }}(\hat{z})-\frac{\epsilon}{4} .
$$

This is a contradiction. Q.E.D.

A natural question to ask is whether $g_{\text {can }}=\frac{i}{2} \partial \bar{\partial} \varphi_{\text {can }}$ defines a complete metric on a bounded strictly pseudoconvex domain in $\mathbf{C}^{n}$ and how it is related to other invariant metrics such as the Bergman, Carathéodory and Kobayashi metrics. 


\section{Further Developments}

As we had acknowledged in the introduction, we could not cover all the possible recent developments, and this survey has not touched on many important topics. In this section, we would like to mention a few and provide some references, for readers who may be completely new to the subject.

A first major omission is a discussion of the important equation $(2.1)$ with $F(z, \varphi)=$ $e^{f(z)-\varphi}$, which corresponds to the open problem of Kähler-Einstein metrics on a compact Kähler manifold $\left(X, \omega_{0}\right)$ with $\omega_{0} \in c_{1}\left(K_{X}^{-1}\right)$. From the discussion of a priori estimates in $\S 6$ and $\S 7$, we see that the equation would be solvable if we can obtain a $C^{0}$ estimate. The problem is to link such an estimate to stability in GIT, as required by the conjecture of Yau [Y93]. Donaldson has recently laid out a program for achieving this [D10, D11a, $\mathrm{D} 11 b, \mathrm{CDa}, \mathrm{CDb}]$. Prior to this program, Kähler-Einstein metrics with positive scalar curvature have been found in various geometric situations by Tian and Yau [TY87] and Tian [T87] using the $\alpha$-invariant, and by Siu [Si] and Nadel [N] using multiplier ideal sheaves. Necessary conditions for Kähler-Einstein metrics have been obtained by Tian [T97]. It has been shown by Tian [T90b] that, for surfaces, the existence of KählerEinstein metrics is equivalent to the vanishing of the Futaki invariant. A full account of the arguments in [T90b] can be found in the paper of Tosatti [T10]. The same characterization of the existence of Kähler-Einstein metrics by the vanishing of the Futaki invariant has been established by Wang and Zhu [WZ2]. Their proof exploits the fact that the toric potentials on a toric variety satisfy a real Monge-Ampère equation, and the image of their gradients is the polytope of the variety. For a survey of some of these developments and the related issue of stability, see [PS03, PS08].

The related question of singularities for the Monge-Ampère equation when the manifold is unstable is of similar considerable interest, and even less explored. The case of holomorphic vector bundles has seen remarkable progress, with the recent works of G. Daskalopoulos and R. Wentworth for complex surfaces [DW1, DW2], and A. Jacob [J1, J2] for general Kähler manifolds, on the generalization to arbitrary dimensions of the AtiyahBott formula for complex curves. An analysis of a break-up of an unstable ruled surface by the Calabi flow has been given by G. Szekelyhidi [Sz].

Another major omission is parabolic complex Monge-Ampère equations, and particularly the Kähler-Ricci flow. As we had mentioned earlier, starting with the papers of Cao [Cao] and Tsuji [Ts], there has been a constant feedback between developments for the elliptic and for the parabolic Monge-Ampère equation. In fact, much of the material discussed in Sections 3, 6, 7 either arose from or are directly motivated by the study of the Kähler-Ricci flow on Fano manifolds (e.g. [ST09, PS06a, PSSW1, CZ, MS, Yu, Zh] and references therein) or on manifolds of general type (e.g. [ST06, TZ, ST09, SW1]). New powerful techniques have been introduced by Perelman (see [ST] for an account of Perelman's unpublished results on the Kähler-Ricci flow). We refer to the papers we listed 
as well as to the recent survey [SW2] for a fuller list of references. Related developments for the Sasaki-Ricci flow can be found in [Co1, Co2, He1].

The Monge-Ampère measure is uniquely defined by Bedford and Taylor [BT76] for locally bounded potentials. The largest classes of possibly unbounded potentials for which a well-behaved measure can be defined have been identified by Cegrell [Ceg] and Blocki [B06]. The Monge-Ampère measures can also be defined for unbounded potentials, if their singularity set is relatively compact within Stein neighborhoods [D, Sib]. A prime example is the pluricomplex Green's function (see e.g. [L, BD, Gb, B00, Ze1] and references therein). As we saw in Section $\S 9$, a non-pluripolar definition can be given, and the range of the corresponding Monge-Ampère measures has been completely characterized by Guedj and Zeriahi [GZ]. The investigation of Monge-Ampère measures which charge pluripolar sets is still in its infancy, see [CG] for examples on projective spaces and [ACCH] for some general results. It is an important direction for research.

In Section §13, we have seen how geodesics in the space of Kähler metrics lead to the homogeneous complex Monge-Ampère equation. Similarly, Donaldson [D07] has shown how geodesics in the space of volume forms on a Riemannian manifold lead to a non-linear equation now known as Donaldson's equation. He also showed how this equation can be interpreted as a PDE version of Nahm's equation in mathematical physics, and is closely related to well-known free boundary problems. The existence of $C^{1, \alpha}$ solutions of Donaldson's equation has been obtained by Chen and $\mathrm{He}[\mathrm{CH}]$ and $\mathrm{He}[\mathrm{H}]$. The same questions of regularity and maximum rank arise for this equation as they do for the homogeneous complex Monge-Ampère equation. Some early results in low dimensions can be found in $[\mathrm{GPa}, \mathrm{GPb}]$. The existence and regularity of geodesics in the space of Sasaki metrics have also been investigated in [GZ].

We have seen in Section 3.2 how the most basic Alexandrov-Bakelman-Pucci estimates can be applied to the complex Monge-Ampère equations. It would be interesting to find out whether this method can be carried out further. In this context, we would like to mention the recent remarkable ABP estimates on Riemannian manifolds obtained by Wang and Zhang [WZ1], building on earlier works of Cabré [Ca1].

Finally, we would like to mention viscosity methods. They have been very successful in the investigation of non-linear equations where no complex structure plays a particular role [CIL]. Even though the notion of plurisubharmonicity poses a number of difficulties, it may not be unreasonable to expect that viscosity methods can be developed and become of wider use for equations such as the complex Monge-Ampère equation. Some major steps in incorporating plurisubharmonicity in viscosity methods have been undertaken by Harvey and Lawson [HL1, HL2], Eyssidieux, Guedj, and Zeriahi [EGZ10], and Wang [W1]. For example, a version of Theorem 12, establishing the existence and uniqueness of viscosity solutions to the Dirichlet problem for the equation (2.1) on domains in $\mathbf{C}^{n}$ for continuous data, has been established in [W1]. 


\section{A Plurisubharmonic functions}

We gather here for the convenience of the reader some basic properties of plurisubharmonic functions and of their Monge-Ampère measures.

\section{A.1 The exponential estimate}

Now the $L^{2}$ norm, in fact the $L^{p}$ norm for any $p<\infty$, of any non-positive plurisubharmonic function $\varphi$ is bounded by a constant depending only on the Kähler class of $\omega_{0}$. This is a consequence of the following local estimate of Hörmander, extended to Kähler manifolds by [T87, TY87, Ze]

Theorem 31 Let $\omega_{0}$ be a Kähler form. There exists a a strictly positive number $\alpha$ and a constant $C$ depending only on $\omega_{0}$ so that

$$
\frac{1}{\left[\omega_{0}^{n}\right]} \int_{X} e^{-\alpha\left(u-\sup _{X} u\right)} \omega_{0}^{n} \leq C
$$

for all $u \in P S H\left(X, \omega_{0}\right)$.

Since $e^{\alpha t} \geq\left(\frac{\alpha}{p}\right)^{p} t^{p}$ for all $t \geq 0$ and all $p>0$, it follows that $\left\|u-\sup _{X} u\right\|_{L^{p}}$ is bounded by a constant depending only on $p$ and $\omega_{0}$.

For recent advances on exponential estimates for plurisubharmonic functions, see [DNS].

\section{A.2 Regularization of plurisubharmonic functions}

The existence of approximations of $\omega_{0}$-plurisubharmonic functions by monotone sequences of smooth $\omega_{0}$-plurisubharmonic functions is much more delicate for Kähler manifolds than for domains in $\mathbf{C}^{n}$. Part of the difficulty resides in the conflicting roles of the differential geometric and the complex structure. An early approximation theorem with loss of $\varepsilon$ positivity is due to Demailly [D89]. Many others are now available, including the recent ones of Demailly, Peternell, and Schneider [DPS] and of Demailly and Paun [DP2], which imply in particular the following statement: let $\left(X, \omega_{0}\right)$ be a Kähler manifold, and let $\gamma$ be a continuous non-negative $(1,1)$-form. Then for any $\varphi \in P S H(X, \gamma)$ with Lelong numbers $\nu_{\varphi}(z)=0$ for all $z \in X$, and any subset $X^{\prime} \subset X$ with compact closure, there exists a decreasing sequence $\varepsilon_{j} \downarrow 0$, and a sequence $\varphi_{j} \in P S H\left(X, \gamma+\varepsilon_{j} \omega_{0}\right) \cap C^{\infty}(X)$ with $\varphi_{j} \downarrow \varphi$ in a neighborhood of $X^{\prime}$. This statement was given an independent proof by Blocki and Kolodziej $[\mathrm{BK}]$. But particularly important for our purposes is the observation of Blocki and Kolodziej [BK] that, for $X$ compact and $\gamma=\omega_{0}$, no loss of positivity is necessary:

Theorem 32 Let $\left(X, \omega_{0}\right)$ be a compact Kähler manifold. Then for every $\varphi \in P S H\left(X, \omega_{0}\right)$, there exists a sequence $\varphi_{j} \in P S H\left(X, \omega_{0}\right) \cap C^{\infty}(X)$ with $\varphi_{j} \downarrow \varphi$. 
For the convenience of the reader, we provide some details on how to derive Theorem 32 from the result of [DPS, DP2].

Let $\varphi \in P S H\left(X, \omega_{0}\right), \varphi \leq-1$ on $X$. For each $j$, the function $\max (\varphi,-j)$ is bounded, and hance has vanishing Lelong numbers. Thus the result of [DPS, DP2] implies the existence of a sequence of smooth functions $\psi_{j k} \in \operatorname{PSH}\left(X,\left(1+\varepsilon_{j, k}\right) \omega_{0}\right)$ with $\psi_{j, k} \downarrow \max (\varphi,-j)$ and $\varepsilon_{j, k} \downarrow 0$ as $k \rightarrow \infty$. By passing to a subsequence, we may assume that $\varepsilon_{j, k} \geq \varepsilon_{j+1, k}$ for all $j, k$. It suffices to show that there exists a sequence $k_{1}<k_{2}<\cdots$ so that $\tilde{\varphi}_{j} \equiv \psi_{j, k_{j}}+\frac{1}{2^{j}}$ is a decreasing sequence converging to $\varphi$. The sequence

$$
\varphi_{j} \equiv \frac{\tilde{\varphi}_{j}}{1+\varepsilon_{j, k_{j}}}
$$

is then a sequence of smooth functions in $\operatorname{PSH}\left(X, \omega_{0}\right)$ with $\varphi_{j} \downarrow \varphi$.

We choose $k_{j}$ inductively as follows. Fix $j$. Let

$$
C_{j}=\left\{\psi_{j+1, k} \geq \psi_{l, k_{l}+j}+\frac{1}{2^{j+1}} \text { for some } l \leq j\right\} .
$$

Since $\cap_{k} C_{k}=\emptyset$, we can choose $k_{j+1}$ so that $C_{k_{j+1}}=\emptyset$. Note that

$$
\psi_{j+1, k_{j}} \leq \psi_{j, k_{j}}+\frac{1}{2^{j+1}} \text { and so } \tilde{\varphi}_{j+1} \leq \tilde{\varphi}_{j}
$$

Now fix $x \in X, \varepsilon>0$, and assume that $\varphi(x) \geq-j_{0}$. Choose $j$ so that

$$
0 \leq \psi_{j_{0}, k_{j}+j}(x)-\varphi(x)<\varepsilon
$$

Then $\psi_{j, k_{j}}-\varphi(x)<\varepsilon+\frac{1}{2^{j}}$, which implies $\tilde{\varphi}_{j}(x)-\varphi(x)<\varepsilon+\frac{1}{2^{j}}+\frac{1}{2^{j}}$. The argument is complete.

\section{A.3 The comparison principle}

The following is a useful version of the comparison principle. It follows from the standard arguments of Bedford and Taylor [BT82], using the above approximation theorem for plurisubharmonic functions on Kähler manifolds.

Theorem 33 Let $\left(X, \omega_{0}\right)$ be a compact Kähler manifold with smooth boundary $\partial X$ and dimension $n$, and let $\omega$ be a smooth, non-negative, closed $(1,1)$-form. Then we have

$$
\int_{\{\varphi<\psi\}}\left(\omega+\frac{i}{2} \partial \bar{\partial} \psi\right)^{n} \leq \int_{\{\varphi<\psi\}}\left(\omega+\frac{i}{2} \partial \bar{\partial} \varphi\right)^{n}
$$

for all $\varphi, \psi \in P S H(X, \omega) \cap L^{\infty}(X)$ satisfying $\liminf _{z \rightarrow \partial X}(\varphi(z)-\psi(z)) \geq 0$. 


\section{Acknowledgements}

The first-named author would like to thank Professors Huai-Dong Cao and Xiaofeng Sun of Lehigh University for their very warm hospitality during his visit there during the 2010 conference in honor of Professor C.C. Hsiung. The authors would like to thank Professor Pengfei Guan for providing them with some invaluable references. They have also benefitted greatly from interactions with Professors O. Munteanu, G. Szekelyhidi, V. Tosatti, Ben Weinkove, and the participants of the informal Complex Geometry and PDE seminar at Columbia University. 


\section{References}

[ACCH] Ahag, P., Cegrell, U., Czyz, R. and Hiep, P.H., Monge-Ampère measures on pluripolar sets, J. Math. Pures Appl. 92 (2009) 613-627.

[AT] Arezzo, C. and Tian, G., Infinite geodesic rays in the space of Kähler potentials, Ann. Sc. Norm. Super. Pisa Cl. Sci. (5) 2 (2003), no. 4, 617630.

[A] Aubin, T., Equations du type Monge-Ampère sur les variétés Kähleriennes compacts, Bull. Sc. Math. 102 (1976), 119-121.

[B] Bedford, E., Survey of pluripotential theory, in Several Complex Variables, ed. J.E. Fornaess, Mittag-Leffler Institute, 1987/1988, Princeton University Press.

[BD] Bedford, E. and J.-P. Demailly, Two counterexamples concerning the pluri-complex Green function in $\mathbf{C}^{n}$, Indiana Univ. Math. J. 37 (1988), 865867.

[BT76] Bedford, E. and Taylor, B.A. The Dirichlet problem for the complex Monge-Ampère operator, Invent. Math. 37 (1976), 1-44.

[BT82] Bedford, E. and Taylor, B.A., A new capacity for plurisubharmonic functions, Acta Math. 149 (1982), 1-40.

[BB] Berman, R. and Boucksom, S., Growth of balls of holomorphic sections and energy at equilibrium, Invent. Math. 181 (2010), no. 2, 337-394.

[BBGZ] Berman, R., Boucksom, S., Guedj, V., and Zeriahi, A. A variatonal approach to complex Monge-Ampère equations, arXiv:0907.4490.

[BD] Berman, R. and Demailly, J.P. Regularity of plurisubharmonic upper envelopes in big cohomology classes, preprint, arXiv:0905.1246.

[Be1] Berndtsson, B., Positivity of direct image bundles and convexity on the space of Kähler metrics J. Differential Geom. 81 (2009), 457-482.

[Be2] Berndtsson, B., Probability measures associated with geodesics in the space of Kähler metrics, preprint, arXiv:0907.1806.

[B00] Blocki, Z., The $C^{1,1}$ regularity of the pluricomplex Green function, Michigan Math. J. 47 (2000) 211-215

[B06] Blocki, Z., The domain of definition of the complex Monge-Ampère operator, Amer. J. Math. 128 (2006) 519-530.

[B09a] Blocki, Z., A gradient estimate in the Calabi-Yau theorem, Math. Ann. 344 (2009), no. 2,317327 .

[B09b] Blocki, Z., On geodesics in the space of Kähler metrics, to appear in the Proceedings of the "Conference in Geometry" dedicated to Shing-Tung Yau (Warsaw, April 2009). 
[B11a] Blocki, Z., On the uniform estimate in the Calabi-Yau theorem, II, Science China Mathematics, 54 (2011), 1375-1377.

[B11b] Blocki, Z., The Calabi-Yau theorem, to appear in Lecture Notes in Mathematics as a part of the volume Complex Monge-Ampère equations and geodesics in the space of Kähler metrics (ed. V. Guedj).

[BK] Blocki, Z. and S. Kolodziej, On regularization of plurisubharmonic functions on manifolds, Proc. Amer. Math. Soc. 135 (2007), 2089-2093.

[BEGZ] Boucksom, S., P. Eyssidieux, V. Guedj, and A. Zeriahi, Monge-Ampère equations in big cohomology classes Acta Math. 205 (2010), no. 2, 199-262.

[BS] Boutet de Monvel, L. and Sjöstrand, J., Sur la singularité des noyaux de Bergman et de Szegö, Astèrisque 3435 (1976), 123164.

[Ca1] Cabre, X., Nondivergent elliptic equations on manifolds with nonnegative curvature, Comm. Pure Appl. Math., 50 (1997) 623-665.

[Ca] Caffarelli, L., Interior $W^{2, p}$ estimates for solutions of Monge-Ampère equations", Ann. of Math. 131 (1990), 135-150.

[CNS] Caffarelli, L., Nirenberg, L., and Spruck, J., The Dirichlet problem for nonlinear second order elliptic equations. I. Monge-Ampère equations, Comm. Pure Appl. Math. 37, 1984, pp. 369-402.

[CKNS] Caffarelli, L., Kohn, J.J., Nirenberg, L. and Spruck, J., The Dirichlet problem for Nonlinear Second-Order Elliptic Equations. II. Complex Monge-Ampère, and Uniformly Elliptic, Equations, Comm. Pure and Appl. Math. 38 (1985), 209- 252.

[Ca2] Calabi, E., Improper affine hyperspheres and generalization of a theorem by K. Jörgens, Mich. Math. J. 5 (1958) 105-126.

[Cao] Cao, H.D., Deformation of Kähler metrics to Kähler-Einstein metrics on compact Kähler manifolds, Invent. Math. 81 (1985), no. 2, 359-372.

[CZ] Cao, H.D. and Zhu, M., A note on compact Kähler-Ricci flow with positive bisectional curvature, Math. Research Letters 16 (2009) 935-939.

[Cat] Catlin, D., The Bergman Kernel and a Theorem of Tian, Analysis and geometry in several complex variables (G. Komatsu et al., Eds.), Birkhauser, 1999, pp. 123.

[Ceg] Cegrell, U., Pluricomplex energy, Acta Math. 180 (1998), 187-217.

[CP] Cegrell, U. and Persson, P., The Dirichlet problem for the complex Monge-Ampère operator: stability in $L^{2}$, Michigan Math. J. 39 (1992)145151.

[C00] Chen, X.X., The space of Kähler metrics, J. Differential Geom. 56 (2000), no. 2, 189 - 234. 
[C06] Chen, X.X., Space of Kähler metrics III: on the lower bound of the Calabi energy and geodesic distance, arXiv: math.DG / 0606228.

[CDa] Chen, X.X. and Donaldson, S.K., Volume estimates for Kähler-Einstein metrics: the three dimensional case, arXiv:1104.0270

[CDb] Chen, X.X. and Donaldson, S.K., Volume estimates for Kähler-Einstein metrics and rigidity of complex structures, arXiv:1104.4331

[CH] Chen, X.X. and He, W., The space of volume forms, preprint, arXiv:0810.3880.

[CS] Chen, X.X. and Sun, S., Calabi flow, geodesic rays, and uniqueness of constant scalar curvature Kähler metrics, preprint, arXiv:1004.2018.

[CT08] Chen, X.X. and Tang, Y., Test configuration and geodesic rays, Astérisque No. 321 (2008), 139-167.

[CT] Chen, X.X. and Tian, G., Geometry of Kähler metrics and foliations by holomorphic discs, Publ. Math. Inst. Hautes Etudes Sci. No. 107 (2008), 1107.

[CW] Chen, Y.Z. and Wu, L.C., Second order elliptic equations and elliptic systems, Transl. Math. Monographs 174 (1998).

[CY80] Cheng, S.Y. and Yau, S.T., On the existence of a complete Kähler-Einstein metric on non-compact complex manifolds and the regularity of Fefferman's equation, Comm. Pure Appl. Math. 33 (1980) 507-544.

[CY86] Cheng, S.Y. and Yau, S.T., Inequality between Chern numbers of singular Kähler surfaces and characterization of orbit space of discrete group of $S U(2,1)$, Contemporary Math. 49 (1986) 31-44.

[Ch] Cherrier, P., Équations de Monge-Ampère sur les variétés Hermitiennes compactes, Bull. Sc. Math (2) 111 (1987), 343-385.

[Co1] Collins, T., The transverse entropy functional and the Sasaki-Ricci flow, preprint, arXiv:1103.5720.

[Co2] Collins, T., Uniform Sobolev inequality along the Sasaki-Ricci flow, preprint, arXiv:1104.1151.

[CG] Coman, D. and Guedj, V., Quasi plurisubharmonic Green's functions, J. de Mathématiques Pures et Appliquées Volume 92, Issue 5 (2009) 456-475

[CIL] Crandall, M., Ishii, H and P.L. Lions, Users guide to viscosity solutions of second order partia differential equations, Bull. Amer. Math. Soc. 27 (1992) 167.

[DW1] Daskalopoulos, G. and R. Wentworth, Convergence properties of the Yang-Mills flow on Kähler surfaces, J. Reine Angew. Math. 575 (2004) 69-99. 
[DW2] Daskalopoulos, G. and R. Wentworth, On the blow-up set of the Yang-Mills flow on Kähler surfaces, Math. Z. 256 (2007), no. 2, 301-310.

[D89] Demailly, J.P., Potential theory in several complex variables, Lecture notes, ICPAM, Nice 1989.

[D] Demailly, J.P., Complex analytic and differential geometry, book available on line at author's website.

[D1] Demailly, J.P., Singular Hermitian metrics on positive line bundles, in Complex algebraic varieties (Bayreuth, 1990), Lecture Notes in Math. 1507, Springer, Berlin, 1992, 87104.

[DP] Demailly, J.P. and Pali, N., Degenerate complex Monge-Ampère equations over compact Kähler manifolds, Internat. J. Math. 21 (2010), no. 3, 357405, arXiv:0710.51095.

[DP2] Demailly, J.P. and Paun, M., Numerical characterization of the Kähler cone of a compact Kähler manifold, Ann. of Math. (2) 159 (2004), no. 3, 1247-1274.

[DPS] Demailly, J.P., T. Peternell, and M. Schneider, Pseudo-effective line bundles on compact Kähler manifolds, Internat. J. Math. 12 (2001), 689-741.

[dH] den Hollander, F., Large deviations, Fields Institute Monographs, 14. American Mathematical Society, Providence, RI, 2000.

[Di09] Dinew, S., Uniqueness in $\mathcal{E}(X, \omega)$, J. of Functional Analysis, 256 (2009), 2113-2122.

[Di09a] Dinew, S., An inequality for mixed Monge-Ampère measures, Math. Z. 262 (2009), 1-15.

[DK] Dinew, S. and Kolodziej, S., Pluripotenial estimates on compact Hermitian manifolds, preprint, arXiv:0910.3937.

[DZ] Dinew, S. and Zhang, Z., On stability and continuity of bounded solutions of degenerate complex Monge-Ampère equations over compact Kähler manifolds, Adv. Math. 225 (2010), no. $1,367-388$.

[DZZ] Dinew, S., Xi Zhang, and Xiangwen Zhang The $C^{2, \alpha}$ estimate of complex Monge-Ampère equation, arXiv:1006.4261

[DNS] Dinh, T.C., Nguyen, V.A., and Sibony, N., Exponential estimates for plurisubharmonic functions and stochastic dynamics, arXiv:0801.1983.

[D87] Donaldson, S., Infinite deteminants, stable bundles, and curvature, Duke Math. J. 54 (1987) 231-247.

[D99] Donaldson, S.K., Symmetric spaces, Kähler geometry and Hamiltonian dynamics, Northern California Symplectic Geometry Seminar, Amer. Math. Soc. Transl. Ser. 2, 196, Amer. Math. Soc., Providence, RI, 1999, 13-33.

[D01] Donaldson, S.K., Scalar curvature and projective imbeddings I, J. Differential Geom. 59 (2001) 479-522. 
[D90] Donaldson, S.K., Holomorphic disks and the complex Monge-Ampère equation, J. Symplectic Geometry 1 (2002) no. 2, 171-196.

[D02] Donaldson, S.K., Scalar curvature and stability of toric varieties, J. Differential Geom. 59 (2002) 289-349.

[D04] Donaldson, S.K., Scalar curvature and projective imbeddings II, Quarterly J. Math. 56 (2005) no. 3, 345-356.

[D07] Donaldson, S.K., Nahm's equations and free-boundary problems, The many facets of geometry, 71-91, Oxford Univ. Press, Oxford, 2010.

[D08] Donaldson, S.K., Constant scalar curvature metrics on toric surfaces, arXiv: 0805.0128

[D10] Donaldson, S.K., Stability, birational transformations and the Kähler-Einstein problem, arXiv:1007.4220

[D11a] Donaldson, S.K., Kähler metrics with cone singularities along a divisor, arXiv:1102.1196

[D11b] Donaldson, S.K., b-Stability and blow-ups, arXiv:1107.1699

[DE] Dupuis, P. and Ellis, R. S., A weak convergence approach to the theory of large deviations, Wiley Series in Probability and Statistics: Probability and Statistics, A Wiley-Interscience Publication. John Wiley \& Sons, Inc., New York, 1997.

[EGZ] Eyssidieux, P., Guedj, V.and Zeriahi, A., Singular Kähler-Einstein metrics, J. Amer. Math. Soc., 22 (3) (2009) 607639.

[EGZ09] Eyssidieux, P., V. Guedj and A. Zeriahi, A priori $L^{\infty}$-estimates for degenerate complex Monge-Ampère equations, Int. Math. Res. Not. IMRN 2008, Art. ID rnn 070, 8 pp.

[EGZ10] Eyssidieux, P., Guedj, V. and A. Zeriahi, A., Viscosity solutions to degenerate complex Monge-Ampère equations, arXiv:1007.0076v1, 2010.

[F] Fefferman, C., Monge-Ampère equations, the Bergman kernel, and geometry of pseudoconvex domains, Ann. of Math. (2) 103 (1976), no. 2, 395-416

[F76] Fefferman, C., "Monge-Ampère equations, the Bergman kernel, and geometry of pseudoconvex domains", Ann. of Math. 103 (1976) 395-416

[FN] Fornaess, J. E. and Narasimhan, R., The Levi problem on complex spaces with singularities, Math. Ann. 248 (1980), no. 1, 4772.

[GS] Gamelin, T. and Sibony, N., Subharmonicity for uniform algebras, J. Funct. Anal. 35 (1980), no. 1, 64-108.

[GT] Gilbarg, D. and Trudinger, N., Elliptic partial differential equations of second order, Classics in Mathematics. Springer-Verlag, Berlin, 2001. xiv+517 pp. ISBN: 3-540-41160-7. 
[Gm] Gill, M., Convergence of the parabolic complex Monge-Ampère equation on compact hermitian manifolds, arXiv:1009.5756.

[Gb] Guan, B., The Dirichlet problem for complex Monge-Ampère equations and regularity of the pluricomplex Green's function, Comm. in Anal. Geom., 8:213218, 2000.

[Gb98] Guan, B., The Dirichlet problem for Monge-Ampère equations in non-convex domains and space-like hypersurfaces of constant Gauss curvature, Trans. Amer. Math. Soc. 350 (1998) 4955-4971

[GL] Guan, B. and Li, Q., Complex Monge-Ampère equations and totally real submanifolds, Adv. Math. (2010), 1185-1223.

[GS] Guan, B. and J. Spruck, Boundary value problem on $S^{n}$ for surfaces of constant Gauss curvature, Annals of Math., 138 (1993), 601-624

[Gd] Guan, D., On modifed Mabuchi functional and Mabuchi moduli space of Kähler metrics on toric bundles, Math. Res. Lett. 6 (1999), no. 5-6, 547-555.

[Gp] Guan, P., A gradient estimate for complex Monge-Ampère equation, 2008 preprint.

[GPa] Guan, P. and Phong, D.H., A maximum rank problem for degenerate elliptic fully nonlinear equations, preprint, arXiv:1009.3975.

[GPb] Guan, P. and Phong, D.H., Partial Legendre transforms of non-linear equations, preprint, arXiv:1010.2111.

[GZ] Guan, P. and Xi Zhang, Regularity of the geodesic equation in the space of Sasakian metrics, arXiv:0906.5591

[GZ] Guedj, V., Zeriahi, A., The weighted Monge-Ampìe energy of quasiplurisubharmonic functions, J. Funct. Anal. 250 (2007), 442-482.

[H] Han, Q., Dirichlet problems of Monge-Ampère equations, lecture notes.

[Ha] Hanani, A. Equations du type de Monge-Ampère sur les variétés hermitiennes compactes, J. Funct. Anal. 137 (1996), no. 1, 4975.

[HL1] Harvey, F.R. and Lawson, H.B., Dirichlet duality and the nonlinear Dirichlet problem, Comm. Pure Appl. Math., 62 (2009) 396-443.

[HL2] Harvey, F.R. and Lawson, H.B., Dirichlet duality and the nonlinear Dirichlet problem on Riemannian manifolds, J. Differential. Geom. 88 (2011) 395-482.

[He1] He, W., The Donaldson equation, preprint, arXiv:0810.4123.

[He2] He, W., Entire solutions of Donaldson's equation, preprint, arXiv:1002.4851.

[Ho] Hörmander, L., An introduction to complex analysis in several variables, North-Holland, 1984. 
[J1] Jacob, A., The limit of the Yang-Mills flow on semi-stable bundles, arXiv:1104.4767.

[J2] Jacob, A., The Yang-Mills flow and the Atiyah-Bott formula for compact Kähler manifolds, arXiv:1109.1550.

[K83] Kobayashi, R., Kähler-Einstein metrics on an open algebraic manifold, Osaka. J. Math. 21 (1984) 399-418

[K98] Kolodziej, S., The complex Monge-Ampère equation, Acta Math. 180 (1998) 69117.

[K03] Kolodziej, S., The Monge-Ampère equation on compact Kähler manifolds, Indiana Univ. Math. J. 52 (2003), no. 3, 667686.

[K05] Kolodziej, S. The complex Monge-Ampère equation and pluripotential theory, Memoirs of AMS, Vol. 178, No. 840, 2005.

[KT] Kolodziej, S. and G. Tian, A uniform $L^{\infty}$ estimate for complex Monge-Ampère equations Math. Ann. 342 (2008), 773-787.

[L] Lempert, L., Solving the degenerate complex Monge-Ampère equation with one concentrated singularity, Math. Ann. 263 (1983) 515-532.

[LV] Lempert, L. and Vivas, L., Geodesics in the space of Kähler metrics, preprint, arXiv:1105.2188.

[LY] Li, P., and Yau, S.T., On the parabolic kernel of the Scrödinger operator, Acta Math. 156 (1986), 153201.

[LYZ] Loftin, J., Yau, S.T., Zaslow, E., Affine manifolds, SYZ geometry and the "Y" vertex, J. Differential Geom. 71 (2005), 129-158.

[L] Lu, Z., On the lower order terms of the asymptotic expansion of Tian-Yau-Zelditch, Amer. J. Math. 122 (2000) 235-273.

[MM] Ma, X., and G. Marinescu, Generalized Bergman kernels on symplectic manifolds, Adv. Math. 217 (2008), 1756-1815.

[M87] Mabuchi, T., Some symplectic geometry on compact Kähler manifolds, Osaka J. Math. 24 (1987) 227-252.

[M08] Mabuchi, T., K-stability of constant scalar curvature polarization, arXiv:0812.4093

[M09] Mabuchi, T., A stronger concept of K-stability, arXiv:0910.4617

[MY83] Mok, N. and Yau, S.T., Completeness of the Kähler-Einstein metric on bounded domains and the characterization of domains of holomorphy by curvature conditions, Proc. Symp. in Pure Math. 39 (1983) 41-59.

[MS] Munteanu, O. and G. Szkelyhidi, On convergence of the Kähler-Ricci flow, arXiv:0904.3505. 
[N] Nadel, A., Multiplier ideal sheaves and Kähler-Einstein metrics of positive scalar curvature, Ann. of Math. 132 (1990) 549-596

[NS] Narasimhan, M.S. and Simha, R.R. Manifolds with ample canonical class, Invent. Math. 5 (1968) $120-128$

[PS03] Phong, D.H. and J. Sturm, Stability, energy functionals, and Kähler-Einstein metrics, Commun. Anal. Geom. 11 (2003) 563-597

[PS06] Phong, D.H. and Sturm, J., The Monge-Ampère operator and geodesics in the space of Kähler potentials, Inventiones Math. 166 (2006) 125-149

[PS06a] Phong, D.H. and Sturm, J., On stability and the convergence of the Kähler-Ricci flow, J. Differential Geom. 72 (2006) no. 1, 149-168

[PS07] Phong, D.H. and Sturm, J., Test configurations and geodesic in the space of Kähler potentials, J. Symplectic Geom. 5 (2007) no. 2, 221-247

[PS07a] Phong, D.H. and Sturm, J., On the regularity of geodesic rays associated to test configurations, arXiv: 0707.3956

[PS08] Phong, D.H. and Sturm, J., Lectures on Stability and Constant Scalar Curvature, in Handbook of Geometric Analysis Vol III, ALM 14, Higher Education Press and International Press, Beijing-Boston, 2009, arXiv: 0801.4179

[PS09a] Phong, D.H. and Sturm, J., The Dirichlet problem for degenerate complex MongeAmpère equations, Commun. Analysis and Geom. 18 (2010) 145-170, arXiv: 0904.1898

[PS09b] Phong, D.H. and Sturm, J., Regularity of geodesic rays and Monge-Ampère equations, Proc. Amer. Math. Soc. 138 (2010), 3637-3650 arXiv: 0908.0556.

[PS09c] Phong, D.H. and Sturm, J., On pointwise gradient estimates for the complex MongeAmpère equation, arXiv:0911.2881, to appear in Conference on Geometry in honor of S.T. Yau, eds. S. Janeczko, J. Li, and D.H. Phong.

[PSS] Phong, D.H., Sesum, N. and Sturm, S., Multiplier ideal sheaves and the Kähler-Ricci flow, Commun. Anal. Geom. 15 (2007), no. 3, 613-632

[PSSW] Phong, D.H., Song, J., Sturm, J. and Weinkove, B., The Moser-Trudinger inequality on Kähler-Einstein manifolds, Amer. J. Math. 130 No. 4 (2008) 1067-1085

[PSSW1] Phong, D.H., Song, J., Sturm, J. and Weinkove, B., The Kähler-Ricci flow and the $\bar{\partial}$ operator on vector fields, J. Differential Geom. 81, 3 (2009), 631647

[PSSW2] Phong, D.H., J. Song, J. Sturm, and Weinkove, B., On the convergence of the modified Kähler-Ricci flow and solitons, Comment. Math. Helv. 86 (2011), no. 1, 91-112. arXiv:0809.0941

[RW] Ross, J. and Witt-Nystrom, D., Analytic test configurations and geodesic rays, arXiv:1101.1612. 
[RZ08] Rubinstein, Y. and Zelditch, Z., Bergman approximations of harmonic maps into the space of Kähler metrics on toric varieties, J. Symplectic Geom. 8 (2010), no. 3, 239265.

[RZ10a] Rubinstein, Y. and Zelditch, Z., The Cauchy problem for the homogeneous MongeAmpère equation, I. Toeplitz quantization, preprint, arXiv:1008.3577.

[RZ10b] Rubinstein, Y. and Zelditch, Z., The Cauchy problem for the homogeneous MongeAmpère equation, II. Legendre transform, preprint, arXiv:1010.2442.

[Se] Semmes, S., Complex Monge-Ampère equations and symplectic manifolds, Amer. J. Math. 114 (1992) 495-550.

[ST09] Sesum, N., and Tian, G., Bounding scalar curvature and diameter along the Kähler Ricci flow (after Perelman), J. Inst. Math. Jussieu 7 (2008), no. 3, 575587.

[Sib] Sibony, N., Quelques problemes de prolongement de courants en analyse complexe, Duke Math. J. Volume 52, Number 1 (1985), 157-197.

[Si] Siu, Y.T., The existence of Kähler-Einstein metrics on manifolds with positive anticanonical line bundle and a suitable finite symmetry group, Ann. of Math. (2) 127 (1988), no. 3, 585627.

[ST06] Song, J. and G. Tian, The Kähler-Ricci flow on surfaces of positive Kodaira dimension, Inventiones Math., 170 Number 3, 609-653

[ST08] Song, J. and Tian, G. Canonical measures and Kähler-Ricci flow, preprint, arXiv:0802.2570.

[SW] Song, J. and B. Weinkove, On the convergence and singularities of the J-flow with applications to the Mabuchi energy, Comm. Pure Appl. Math. 61 (2008), no. 2, 210-229.

[SW1] Song, J. and B. Weinkove, Contracting exceptional divisors by the Kähler-Ricci flow, arXiv:1003.0718

[SW2] Song, J. and B. Weinkove, Lecture Notes on the Kähler-Ricci flow http://www.math.ucsd.edu/ bweinkov/Research.html, http://www.math.rutgers.edu/ jiansong/publication.htm

[SZ07] Song, J. and Zelditch, S., Test configurations, large deviations and geodesic rays on toric varieties, preprint, arXiv:0712.3599.

[SZ10] Song, J. and Zelditch, S., Bergman metric and geodesics in the space or Kähler metrics on toric varieties, Anal. PDE 3 (2010), no. 3, 295-358.

[Sz] Szekelyhidi, G., The Calabi functional on a ruled surface, arXiv: math/0703562

[ST] Szekelyhidi, G. and V. Tosatti, Regularity of weak solutions of a complex Monge-Ampre equation, arXiv:0912.1808 
[T87] Tian, G., On Kähler-Einstein metrics on certain Kähler manifolds with $C_{1}>0$, Comm. Math. Phys. 112 (1987), no. 1, 175-203.

[T90a] Tian, G., On a set of polarized Kähler metrics on algebraic manifolds, J.Differential Geometry 32 (1990), 99 - 130.

[T90b] Tian, G., On Calabis conjecture for complex surfaces with positive first Chern class, Invent. Math. 101, no. 1 (1990), 101-172.

[T97] Tian, G., Kähler-Einstein metrics with positive scalar curvature, Invent. Math. 130 (1997), no. $1,1-37$.

[TY86] Tian, G. and S.T. Yau, Existence of Kähler-Einstein metrics on complete Kähler manifolds and their applications to algebraic geometry, in Mathematical aspects of string theory, ed. by S.T. Yau (1987) 575-627, World Scientific.

[TY87] Tian, G. and S.T. Yau, Kähler-Einstein metrics on complex surfaces with $c_{1}(M)$ positive, Comm. Math. Phys. 112 (1987) 175-203.

[TY90] Tian, G. and S.T. Yau, Complete Kähler manifolds with zero Ricci curvature I, J. Amer. Math. Soc. 3 (1990) 579-609

[TY91] Tian, G. and S.T. Yau, Complete Kähler manifolds with zero Ricci curvature II, Inventiones Math. 106 (1991) 27-60

[TZ] Tian, G. and Zhang, Z. On the Kähler-Ricci flow on projective manifolds of general type, Chinese Ann. Math. Ser. B 27 (2006), no. 2, 179-192.

[T10] Tosatti, V., Kähler-Einstein metrics on Fano surfaces, preprint, arXiv:1010.1500.

[TW1] Tosatti, V. and B. Weinkove, Estimates for the complex Monge-Ampère equation on Hermitian and balanced manifolds, Asian J. Math. 14 (2010), no. 1, 19-40.

[TW2] Tosatti, V. and B. Weinkove, The complex Monge-Ampère equation on compact Hermitian manifolds, J. Amer. Math. Society 23 no. 4 (2010) 1187-1195.

[TWY] Tosatti, V., Weinkove, B. and Yau, S.T., Taming symplectic forms and the Calabi-Yau equation, Proc. Lond. Math. Soc. (3) 97 (2008), no. 2, 401-424.

[TrWa] Trudinger, N. and X.J. Wang, Boundary regularity for the Monge-Ampère and affine maximal surface equations, Ann. of Math. 167 (2008), 993-1028.

[Ts] Tsuji, H., Existence and degeneration of Kähler-Einstein metrics on minimal algebraic varieties of general type, Math. Ann. 281 (1988), 123-133.

[Ts07] Tsuji, H., Canonical singular hermitian metrics on relative canonical bundles, preprint, arXiv:0704.0566.

[UY] Uhlenbeck, K. and Yau, S.T., On the existence of Hermitian Yang-Mills connections on stable vector bundles, Commun. Pure Appl. Math. 39 (1986) 257-293. 
[V] Varadhan, S.R.S., Asymptotic probability and differential equations, Comm. Pure Appl. Math. 19, 261- 286.

[Wg] Wang, X., Canonical metrics on stable vector bundles, Comm. Anal. Geom. 13 (2005), 253-285.

[W1] Wang, Y., A viscosity approach to the Dirichlet problem for complex Monge-Ampère equations, arXiv: 1010.1292.

[W2] Wang, Y., A remark on $C^{2, \alpha}$-regularity of the complex Monge-Ampère equation, arXiv:1111.0902.

[WZ1] Wang, Y. and Zhang, X., Measure Estimates, Harnack Inequalities and Ricci Lower Bound, arXiv:1102.5567.

[WZ2] Wang, X.J. and Zhu, X.H., Kähler-Ricci solitons on toric manifolds with positive first Chern class, Advances Math. 188 (2004) 87-103.

[W] Weinkove, B., Convergence of the J-flow on Kähler surfaces, Comm. Anal. Geom. 12 (2004), no. 4, 949-965.

[W08] Wu, D., Kähler-Einstein metrics of negative Ricci curvature on general quasi-projective manifolds, Commun. Analysis Geom. 16 (2008) 395-435

[Y75] Yau, S.T., Harmonic functions on complete Riemannian manifolds, Commun. Pure Appl. Math. 28 (1975), 201228.

[Y78] Yau, S.T., On the Ricci curvature of a compact Kähler manifold and the complex MongeAmp'ere equation, I, Comm. Pure Appl. Math., 31 (1978), 339 - 411.

[Y78a] Yau, S.T., A general Schwarz lemma for Kähler manifolds, Amer. J. Math. 100 (1978), no. 1, 197-203.

[Y93] Yau, S.T., Open problems in geometry, Proc. Symposia Pure Math. 54 (1993) 1-28.

[Y94] Yau, S.T., A survey of Kähler metrics, Proc. Symp. in Pure Math. 41 (1984) 285-289.

[Y96] Yau, S.T., Review of Kähler-Einstein metrics in algebraic geometry, Israel Math. Conference Proc. 9 (1996) 433-443

[Z] Zelditch, S., Szegö kernels and a theorem of Tian, Int. Math. Res. Notices, no.6 (1998), 317-331.

[Ze] Zeriahi, A., Volume and capacity of sublevel sets of a Lelong class of plurisubharmonic functions, Indiana Univ. Math. J. 50 (2001), 671-703.

[Ze1] Zeriahi, A., Pluricomplex Green functions and the Dirichlet problem for the complex MongeAmpère operator, Michigan Math. J. 44 (1997) 589-596.

[Yu] Yuan, Y., On the convergence of a modified Kähler-Ricci flow, to appear in Math. Zeitschrift. 
[Zh] Zhang, Z.L. Kähler Ricci flow on Fano manifolds with vanished Futaki invariants, arXiv:1010.5959, to appear in Math. Research Letters.

[ZZh] Zhang, Zhou, On degenerate Monge-Ampre equations over closed Kähler manifolds, Int. Math. Res. Not., Vol. 2006, Art. ID 63640, Pages 1 - 18.

[ZZ] Zhang, Xi and XiangWen Zhang, Regularity estimates of solutions to complex Monge-Ampre equations on Hermitian manifolds, arXiv:1007.2627 